\title{
Structure constants of twist-two light-ray operators in the triple Regge limit
}

\section{Balitsky}

Physics Department, Old Dominion University, Norfolk, VA 23529, U.S.A.

Thomas Jefferson National Accelerator Facility, Newport News, VA 23606, U.S.A.

E-mail: balitsky@jlab.org

ABSTRACT: The structure constants of twist-two operators with spin $j$ in the BFKL limit $g^{2} \rightarrow 0, j \rightarrow 1$ and $\frac{g^{2}}{j-1} \sim 1$ are found from the calculation of the three-point correlator of twist-two light-ray operators in the triple Regge limit. It is well known that the anomalous dimensions of twist-two operators in this limit are determined by the BFKL intercept. Similarly, the obtained structure constants are determined by an analytic function of three BFKL intercepts.

KEYwords: Resummation, Perturbative QCD, Supersymmetric Gauge Theory, Conformal and W Symmetry

ARXIV EPRINT: 1812.07044 


\section{Contents}

1 Introduction 1

2 3-point correlators and structure constants of "forward" operators 4

3 Light-ray operators in the BFKL limit $\quad \mathbf{5}$

3.1 Light-ray operators as an analytic continuation of local operators 5

3.2 Correlators of the light-ray operators in the BFKL limit: what to expect 7

4 Wilson frames $\quad 8$

5 Correlator of two Wilson frames in the BFKL limit 9

5.1 Rapidity factorization for 4-point correlators in the Regge limit 10

5.2 Correlator of two Wilson frames in the Regge limit 12

6 Correlator of three Wilson frames in the triple Regge limit 18

$\begin{array}{lll}6.1 \text { Triple BFKL evolution } & 18\end{array}$

$\begin{array}{ll}6.2 \text { Longitudinal integrals } & 23\end{array}$

6.3 Transverse integral 24

$\begin{array}{ll}6.4 \text { The result } & 27\end{array}$

$\begin{array}{llr}7 & \text { Conclusions } & 30\end{array}$

A BFKL kernel in the triple Regge limit 31

B Correlator of three twist-2 LR operators in the tree approximation $\quad 35$

$\begin{array}{ll}\text { C Boost invariance and singularities of structure constants } & 38\end{array}$

D Calculation of $\Lambda\left(\nu_{1}, \nu_{2}, \nu_{3}\right) \quad 42$

\section{Introduction}

The approximate conformal invariance of pQCD makes it very useful in practical calculations. Any leading-order pQCD result which does not have explicit $\beta$-function can be obtained from conformally invariant amplitudes. Moreover, the results obtained in a close conformal "neighbor" of QCD, the $\mathcal{N}=4$ SYM theory, can be used as a starting point of QCD calculation. Typically, the result in $\mathcal{N}=4$ theory gives the most complicated part of pQCD result, i.e. the one with maximal transcendentality. This is explicitly confirmed in many cases, for example in the calculation of anomalous dimensions of twist-two operators [1,2] and cusp anomalous dimension at the three-loop level [3, 4]. Actually, 
it is worthwhile to start a pQCD calculation from the corresponding analysis in $\mathcal{N}=4$ SYM. The Lagrangian might seem more complicated but the result will be obtained in a more streamlined and controlled way and it will give the most transcendental part of the QCD result.

It is well known that the all correlation functions (correlators) in a conformal theory are in principle determined if one knows the anomalous dimensions of all primary operators and the corresponding structure constants determined by three-point correlators. The important class of local operators is represented by the so-called twist-two operators encountered in many phenomenological applications in QCD starting from the famous case of deep inelastic scattering. As to anomalous dimensions of twist-two operators in $\mathcal{N}=4$ SYM, there was a considerable progress in recent years due to the development of QCS method $[5,6]$ resulting in analytic expressions at large $N_{c}$ up to 7 th order of perturbation theory [7] and very accurate numerical calculations at any coupling constant up to a strong coupling limit [8].

In contrast, the study of structure constants of twist-two operators is not at the same level yet. For arbitrary spins, the structure constants of three twist-two operators are explicitly known only at the tree level $[9,10]$. There are calculations of the structure constants of two protected operators and a twist-two operator, the most recent performed using the hexagon approach [11] up to the four-loop [12] and five-loop [13] level. However, for the correlator of three non-protected operators the hexagon approach gives only general prescription for calculations and to get explicit results further development of hexagon method is necessary. There is also a related QCS calculation of three-cusp Wilson loop similar to correlator of one protected and two non-trivial operators [14], but at this stage it is not clear whether such result can be used to get the correlator of three twist-two operators.

In this circumstances, it is very useful to find examples of explicit calculation of twisttwo structure constants, especially in the approximations which go beyond the leading orders of perturbation theory. One of the most interesting examples is the structure constants of twist-two operators in the so-called BFKL limit when the Lorentz spin of the twist-two operator tends to one: $\omega=j-1 \rightarrow 0$, coupling constant $g^{2}=\frac{g_{\mathrm{YM}}^{2}}{16 \pi^{2}} N_{c}$ is small but the ratio $\frac{g^{2}}{\omega}$ is fixed. This limit is closely related to the high-energy behavior of amplitudes, roughly speaking $\frac{g^{2}}{\omega} \sim g^{2} \ln E$ where $E$ is the energy. The problem of high-energy behavior of amplitudes has a long story starting with Heisenberg-Froissart bound $\ln ^{2} E$ for total cross section which has not been constructively explained in any (field or string) theory in more than 50 years. The most popular idea is to reduce the gauge theory at high energies to $2+1$ effective theory which can be solved (exactly or by computer simulations). Unfortunately, despite the multitude of attempts, the Lagrangian for $2+1$ QCD or $\mathcal{N}=4$ SYM at high energies is not written yet. In this context, the complementary approach of conformal bootstrap may be helpful. One may start with twist-two operators in the BFKL limit and use knowledge of anomalous dimensions and structure constants of these operators to construct the high-energy amplitudes. Of course, the high-energy behavior of amplitudes is not completely determined by twist-two operators, for example the BFKL equation involves twist two as well as all higher twists. Still, the effective conformal theory of twist-two operators at small $\omega$ 's appears to be a good place to start. 
Since the conformal twist-two operator in $\mathcal{N}=4$ SYM looks like

$$
\mathcal{O}^{j}(x)=\operatorname{Tr} F_{+i} D_{+}^{j-2} F_{+}{ }^{i}+\text { gluinos }+ \text { scalars }
$$

the point $\omega=j-1 \rightarrow 0$ is an unorthodox point corresponding to the non-local operator $\operatorname{Tr} F_{+i} D_{+}^{\omega-1} F_{+}^{i}$. The explicit form of this non-local operator is a so-called light-ray operator - a bilocal operator with the light-like gauge link. Such light-ray operators are extensively studied in QCD since matrix elements of those operators define parton distribution densities for forward case and so-called GPDs in the off-forward case (see the book [15] for a review). For $\mathcal{N}=4 \mathrm{SYM}$, the supersymmetric generalization of QCD light-ray operators [16] is presented in ref. [17] following the corresponding work on the supermultiplet of twist-two local operators [18].

The anomalous dimensions of twist-two operators in the BFKL limit can be obtained from Regge asymptotics of the four-point correlators resulting in the equation $\omega=\aleph(\Delta)$ where $\Delta$ is the dimension of the operator and $\aleph(\Delta)$ is the famous Pomeron intercept. In QCD, it is known only up to the NLO order [19], but in $\mathcal{N}=4$ SYM it is studied well beyond that: there are explicit perturbative expressions at the NNLO level [20-22], numerical estimates at few extra orders [20] and several terms for the large-coupling expansion around graviton point $j=2[8,23-25]$.

Thus, the theory of anomalous dimensions of twist-two operators in the BFKL limit seems to be well developed and it would be very interesting to bring the study of structure constants up to the same level. The most direct way to find the structure constants in the BFKL limit is to compute the correlation function of the corresponding three lightray operators. This was done in refs. [26, 27] using the non-linear evolution equation for color dipoles [28-30] and the result is that the structure constant is determined by so-called three-pomeron vertex [31] projected onto Lipatov's eigenfunctions of the BFKL kernel [32]. However, by this method it is possible to obtain structure constants only at $\omega_{1}=\omega_{2}+\omega_{3}$ and generalization to arbitrary $\omega$ 's requires the analysis of perturbative diagrams in the triple Regge limit. It should be noted that the triple Regge limit is a somewhat novel regime of resummation in perturbation theory. Roughly speaking, it describes the interaction of three particles going with the speed near speed of light along $x, y$, and $z$ directions. Such limit was not studied in QCD (or any other QFT) except for ref. [33] devoted to possible anomaly coming from three pomerons interacting by quark exchange (in our LLA calculations quark exchanges are neglected since they are subleading at high energies).

In this paper, following the logic of earlier papers [17, 26, 27], I calculate the correlator of three light-ray operators (1.1) in the triple BFKL limit $g^{2}, \omega_{i} \rightarrow 0$ and $\frac{g^{2}}{\omega_{i}} \sim 1$ in the leading logarithmic approximation (LLA). The three light rays are collinear to three linearly independent light-like vectors $n_{1}, n_{2}$, and $n_{3}$. To simplify the complicated spin structure of a general correlator of three light-ray operators, I place these operators on the same line in the direction orthogonal to all $n_{i}$, and integrate each light-ray operator along the total translation in the corresponding $n_{i}$ direction. As demonstrated in ref. [27], the resulting correlator has only one tensor structure and computing the coefficient in front of that structure is the aim of this paper. Moreover, since it is well known that in the 
LLA-Regge limit the contributions of gluino and scalar fields are sub-leading, the obtained result for three-point correlator will be valid in QCD as well.

The paper is organized as follows. In section 2 I recall the generic structure of 3 point correlator for local twist-2 operators and present the form of the correlator of three "forward" local operators integrated over the total translation in corresponding light-like directions. This formula is generalized to correlator of three twist-two light-ray operators in section 3. In section 4 I define "Wilson frame" operators and in section 5 remind the calculation of two-point correlator of these operators in the BFKL limit. In section 6 which is central to this paper, I calculate the correlator of three Wilson frames in the BFKL limit and present the result for the structure constant. In the Conclusions section I discuss the obtained result and its relation to the result of ref. [26]. The appendix contains derivations of technical results used in section 6 .

\section{3-point correlators and structure constants of "forward" operators}

The general structure of 3-point correlators of local operators with spin was found in ref. [34] to be ${ }^{1}$

$$
\left\langle\mathcal{O}_{n_{1}}^{j_{1}}(x) \mathcal{O}_{n_{2}}^{j_{2}}(y) \mathcal{O}_{n_{3}}^{j_{3}}(z)\right\rangle=\sum_{m_{12}, m_{13}, m_{23} \geq 0} \lambda_{m_{12}, m_{23}, m_{13}}\left[\begin{array}{ccc}
\Delta_{1} & \Delta_{2} & \Delta_{3} \\
j_{1} & j_{2} & j_{3} \\
m_{23} & m_{13} & m_{12}
\end{array}\right]
$$

where $\mathcal{O}_{n}^{l}(x)$ is a spin- $l$ operator with indices contracted with light-like vector $n$, the square brackets represent some tensor structures and the sum over $m_{i j}$ goes over positive integers satisfying certain inequalities. Following refs. [26] and [27] I consider the correlator of three "forward" operators integrated over corresponding light-like lines

$$
\int d v_{1} d v_{2} d v_{3}\left\langle\mathcal{O}_{n_{1}}^{j_{1}}\left(v_{1} n_{1}+z_{1_{t}}\right) \mathcal{O}_{n_{2}}^{j_{2}}\left(v_{2} n_{2}+z_{2_{t}}\right) \mathcal{O}_{n_{3}}^{j_{3}}\left(v_{3} n_{3}+z_{3_{t}}\right)\right\rangle
$$

where the transverse separations $z_{i_{t}}$ are orthogonal to all $n_{i}$. It has been demonstrated in ref. [27] that after such integration all tensor structures collapse to one and we get:

$$
\begin{aligned}
& \int d v_{1} d v_{2}\left\langle\mathcal{O}_{n_{1}}^{j}\left(v_{1} n_{1}+z_{1_{t}}\right) \mathcal{O}_{n_{2}}^{j^{\prime}}\left(v_{2} n_{2}+z_{2_{t}}\right)\right\rangle=\delta\left(j-j^{\prime}\right) \frac{C(\Delta, j) s_{12}^{j-1}}{\left|z_{12_{t}}^{2}\right|^{\Delta-1}} \mu^{-2 \gamma}, \\
& \int d v_{1} d v_{2} d v_{3}\left\langle\mathcal{O}_{n_{1}}^{j_{1}}\left(v_{1} n_{1}+z_{1_{t}}\right) \mathcal{O}_{n_{2}}^{j_{2}}\left(v_{2} n_{2}+z_{2_{t}}\right) \mathcal{O}_{n_{3}}^{j_{3}}\left(v_{3} n_{3}+z_{3 t}\right)\right\rangle \\
& \quad=C\left(\Delta_{i}, j_{i}\right) \frac{s_{12}{ }^{\frac{j_{1}+j_{2}-j_{3}-1}{2}}}{\left|z_{12_{t}}\right|^{\Delta_{1}+\Delta_{2}-\Delta_{3}-1}} \frac{s_{13}}{\left|z_{13_{t}}\right|^{\Delta_{1}+\Delta_{3}-\Delta_{2}-1}} \frac{s_{23}{ }^{\frac{j_{2}+j_{3}-j_{1}-1}{2}}}{\left|z_{23_{t}}\right|^{\Delta_{2}+\Delta_{3}-\Delta_{1}-1}} \mu^{-\gamma_{1}-\gamma_{2}-\gamma_{3}}
\end{aligned}
$$

where $\mu$ is the normalization point, $s_{i j} \equiv-2 n_{i} \cdot n_{j}, z_{i j} \equiv z_{i}-z_{j}$ and $\Delta_{i}$ are dimensions (canonical $d_{i}$ plus anomalous $\gamma_{i}$ ) of operators $\mathcal{O}_{i}$.

As was mentioned in the Introduction, the most interesting operators for possible phenomenological applications are the twist-two operators. The supermultiplet of twist-2

\footnotetext{
${ }^{1}$ To save space, throughout the paper we use notation $\left\langle\mathcal{O}_{1}\left(x_{1}\right) \ldots \mathcal{O}_{n}\left(x_{n}\right)\right\rangle \equiv\left\langle T\left\{\mathcal{O}_{1}\left(x_{1}\right) \ldots \mathcal{O}_{n}\left(x_{n}\right)\right\}\right\rangle$.
} 
operators in $\mathcal{N}=4 \mathrm{SYM}$ was explicitly constructed in ref. [18]. In our case of "forward" operators it reads

$$
\begin{aligned}
& S_{1}^{j}=\mathcal{O}_{g}^{j}+\frac{1}{2} \mathcal{O}_{\lambda}^{j}-\frac{1}{2} \mathcal{O}_{\phi}^{j}, \quad S_{2}^{j}=\mathcal{O}_{g}^{j}-\frac{1}{2(j-1)} \mathcal{O}_{\lambda}^{j}+\frac{j+1}{6(j-1)} \mathcal{O}_{\phi}^{j} \\
& S_{3}^{j}=\mathcal{O}_{g}^{j}-\frac{j+2}{j-1} \mathcal{O}_{\lambda}^{j}-\frac{(j+1)(j+2)}{2 j(j-1)} \mathcal{O}_{\phi}^{j}
\end{aligned}
$$

where $^{2}$

$$
\begin{aligned}
\mathcal{O}_{\phi}^{j}\left(x_{t}\right) & =\int d u \bar{\phi}_{A B}^{a} \nabla_{n}^{j} \phi^{A B a}\left(u n+x_{t}\right), \\
\mathcal{O}_{\lambda}^{j}\left(x_{t}\right) & =\int d u i \bar{\lambda}_{A}^{a} \nabla_{n}^{j-1} \sigma_{n} \lambda_{A}^{a}\left(u n+x_{t}\right) \\
\mathcal{O}_{g}^{j}\left(x_{t}\right) & =\int d u F_{n i}^{a} \nabla_{n}^{j-2} F_{n}^{a i}\left(u n+x_{t}\right) .
\end{aligned}
$$

The operators (2.3) are multiplicatively renormalized operators with anomalous dimensions

$$
\gamma_{j}^{S_{1}}\left(\alpha_{s}\right)=4\left[\psi(j-1)+\gamma_{E}\right]+O\left(\alpha_{s}^{2}\right), \quad \gamma_{j}^{S_{2}}=\gamma_{j+2}^{S_{1}}, \quad \gamma_{j}^{S_{3}}=\gamma_{j+4}^{S_{1}}
$$

As mentioned in the Introduction, the goal is to calculate the structure constant $C$ in eq. (2.2) in the "triple BFKL limit" $g^{2} \rightarrow 0, \omega_{i}=j_{i}-1 \rightarrow 0$ but $\frac{g^{2}}{\omega_{i}} \sim 1$. However, at $\omega \rightarrow 0$ these gluon operators are no longer local. Instead, they are represented by so-called light-ray operators discussed in the next section.

\section{Light-ray operators in the BFKL limit}

\subsection{Light-ray operators as an analytic continuation of local operators}

Light-ray (LR) operators are defined as bilocal operators with light-like separation and gauge links providing gauge invariance. For example, the gluon light-ray twist-two operator is defined as

$$
F_{\alpha \xi}^{a}(x)[x, y]^{a b} F_{\beta}^{b \xi}(y), \quad(x-y)^{2}=0
$$

where the gauge link $[x, y]$ is defined as

$$
[x, y] \equiv \operatorname{Pexp}\left\{-i g_{\mathrm{YM}} \int_{0}^{1} d u(x-y)_{\mu} A^{\mu}(u x+(1-u) y)\right\}
$$

These operators represent the sum of local operators of twist two convoluted with light-like vector $x-y$. They possess extra UV divergencies in addition to usual self-energy and vertex UV divergencies so they are defined with a set of counterterms and the dependence of this counterterms on the UV cutoff defines the evolution equations for light-ray operators.

\footnotetext{
${ }^{2}$ We use metric $g^{\mu \nu}=(-1,1,1,1)$ and the covariant derivative is $\nabla_{\mu}=\left(\partial_{\mu}-i g_{\mathrm{YM}}\left[A_{\mu},\right)\right.$.
} 
The LR operator (3.1) can be interpreted as an analytic continuation of a local operator to non-integer number of covariant derivatives. Indeed, if we can represent $F_{n \xi}^{a} \nabla_{n}^{j-2} F_{n}^{a \xi}(0)$ as

$$
\begin{aligned}
F_{n \xi}^{a} \nabla_{n}^{j-2} F_{n}^{a \xi}(0) & =\frac{\Gamma(j-1)}{2 \pi i} \int_{C} d u u^{1-j} F_{n \xi}^{a} e^{-u \nabla_{n}} F_{n}^{a \xi}(0) \\
& =\frac{\Gamma(j-1)}{2 \pi i} \int_{C} d u u^{1-j} F_{n \xi}^{a}(u x)[u x, 0]^{a b} F_{n}^{b \xi}(0)
\end{aligned}
$$

where $C$ is the contour of integration in Hankel's formula for gamma-function. ${ }^{3}$ At noninteger $j$ this formula can be simplified to

$$
\begin{aligned}
F_{n \xi}^{a} \nabla_{n}^{j-2} F_{n}^{a \xi}(0) & =\frac{1}{\Gamma(2-j)} \int_{0}^{\infty} d u u^{1-j} F_{n \xi}^{a}(u x)[u x, 0]^{a b} F_{n}^{b \xi}(0) \\
& \Rightarrow \int_{0}^{\infty} d u u^{1-j} F_{n \xi}^{a}(u x)[u x, 0]^{a b} F_{n}^{b \xi}(0)=\Gamma(2-j) F_{n \xi}^{a} \nabla_{n}^{j-2} F_{n}^{a \xi}(0)
\end{aligned}
$$

At $j=-\frac{1}{2}+i \varsigma$ this light-ray operator realizes the principal series irreducible representation of $s l(2 \mid 4)$ with conformal spin $J=j+1=\frac{1}{2}+i \varsigma$. Since it is well-defined at $J=\frac{1}{2}+i \varsigma$ it can be uniquely analytically continued to the whole complex plane of $J$ and the continuation to integer $J=k+1$ gives local operator as a residue in the pole at $j=k$.

The generalization of supermultiplet of twist-two operators (2.3) to the case of complex spin $j$ was constructed in [17]. We defined "forward" parity-even light-ray operators as

$$
\begin{aligned}
\mathcal{F}_{n}^{j}\left(x_{t}\right) & =\int_{0}^{\infty} d l l^{1-j} \mathcal{F}_{n}\left(l, x_{t}\right), \quad \Lambda_{n}^{j}\left(x_{t}\right)=\int_{0}^{\infty} d l l^{-j} \Lambda_{n}\left(l, x_{t}\right) \\
\Phi_{n}^{j}\left(x_{t}\right) & =\int_{0}^{\infty} d l l^{-1-j} \Phi_{n}\left(l, x_{t}\right)
\end{aligned}
$$

where

$$
\begin{aligned}
\mathcal{F}_{n}\left(l, x_{t}\right)=\int d v F_{n \xi}^{a}\left(l n+v n+x_{t}\right)[l+v, v]^{a b} F_{n}^{b \xi}\left(v n+x_{t}\right) \\
\Lambda_{n}\left(l, x_{t}\right)=\frac{i}{2} \int d v\left[-\bar{\lambda}_{A}^{a}\left(\ln +v n+x_{t}\right)[l+v, v]_{x}^{a b} \sigma_{n} \lambda_{A}^{b}\left(v n+x_{t}\right)\right. \\
\left.+\bar{\lambda}_{A}^{a}\left(v n+x_{t}\right)[v, l+v]^{a b} \sigma_{n} \lambda_{A}^{b}\left(\ln +v n+x_{t}\right)\right] \\
\Phi_{n}\left(l, x_{t}\right)=\int d v \phi_{I}^{a}\left(\ln +v n+x_{t}\right)[l+v, v]_{x}^{a b} \phi_{I}^{b}\left(v n+x_{t}\right)
\end{aligned}
$$

where $[u, v]_{x}$ is a shorthand notation for $\left[u n+x_{t}, v n+x_{t}\right]$. The corresponding renorminvariant light-ray operators are given by $[17]$

$$
\begin{aligned}
& S_{1}^{j}=\mathcal{F}_{j}-\frac{j-1}{2} \Lambda_{j}-\frac{1}{2} j(j-1) \Phi_{j}, \quad S_{2}^{j}=\mathcal{F}_{j}+\frac{1}{2} \Lambda_{j}-\frac{j+1}{6} \Phi_{j} \\
& S_{3}^{j}=\mathcal{F}_{j}+(j+2) \Lambda_{j}-\frac{(j+1)(j+2)}{2} \Phi_{j}
\end{aligned}
$$

where the difference between the coefficients here and in eq. (2.3) is due to eq. (3.4).

\footnotetext{
${ }^{3}$ The path of integration starts at $\infty+i 0$ at the real axis, goes to $\epsilon+i 0$, circles the origin in the counterclockwise direction with radius $\epsilon$ to the point $\epsilon-i 0$, and returns to the point $\infty-i 0$.
} 
It is demonstrated in ref. [35] that analytic continuation of anomalous dimensions of local operators eq. (2.3) to non-integer $j$ by integrals of DGLAP kernels gives the anomalous dimensions of light-ray operators (3.7). Consequently, the anomalous dimensions of lightray operators (3.7) are related by the same eq. (2.5) as local operators (2.3).

Since supersymmetric light-ray operators $S^{j}$ are analytic continuation of local operators, one should expect the same formulas as (2.2) for correlators of local operators:

$$
\left\langle S^{j}\left(z_{1 t}\right) S^{j^{\prime}}\left(z_{2 t}\right)\right\rangle=\delta\left(j-j^{\prime}\right) \frac{C(j, \Delta) s_{12}^{j-1}}{\left(z_{12 t}^{2}\right)^{\Delta-1}} \mu^{-2 \gamma}
$$

and

$$
\begin{aligned}
& \left\langle S_{n_{1}}^{j_{1}}\left(z_{1_{t}}\right) S_{n_{2}}^{j_{2}}\left(z_{2_{t}}\right) S_{n_{3}}^{j_{3}}\left(z_{3_{t}}\right)\right\rangle \\
& =C\left(\Delta_{i}, j_{i}\right) \frac{s_{12}^{\frac{j_{1}+j_{2}-j_{3}-1}{2}}}{\left|z_{12_{t}}\right|^{\Delta_{1}+\Delta_{2}-\Delta_{3}-1}} \frac{s_{13}^{\frac{j_{1}+j_{3}-j_{2}-1}{2}}}{\left|z_{13_{t}}\right|^{\Delta_{1}+\Delta_{3}-\Delta_{2}-1}} \frac{s_{23}^{\frac{j_{2}+j_{3}-j_{1}-1}{2}}}{\left|z_{23_{t}}\right|^{\Delta_{2}+\Delta_{3}-\Delta_{1}-1}} \mu^{-\gamma_{1}-\gamma_{2}-\gamma_{3}}
\end{aligned}
$$

Note that from eq. (3.4) we see that the canonical dimension of light-ray operators (3.5) is $j+2$.

As mentioned above, the goal of this paper is to find structure constants of operators $S_{1}^{j}$ in the BFKL limit $g^{2} \rightarrow 0, \omega_{i}=j_{i}-1 \rightarrow 0, \frac{g^{2}}{\omega_{i}} \sim 1$. The important observation is that at small $\omega$ it is sufficient to study the correlator of three gluon operators $\int d u F_{n i}^{a} \nabla_{n}^{\omega-1} F_{n}^{a i}(u n+$ $x_{t}$ ). Indeed, solving eqs. (3.7) we see that

$$
\mathcal{F}_{j}=\frac{S_{1}^{j}+\omega\left(\frac{5+\omega}{6} S_{1}^{j}+\left(6+\frac{13}{2} \omega+\frac{3}{2} \omega^{2}\right) S_{2}^{j}-\frac{5+4 \omega}{6} S_{3}^{j}\right)}{1+6 \omega+6 \omega^{2}+\frac{3}{2} \omega^{3}}
$$

so at small $\omega=j-1$ the operator $S_{1}^{j}$ is approximately equal to gluon operator $\mathcal{F}^{j}$.

\subsection{Correlators of the light-ray operators in the BFKL limit: what to expect}

As we noted above, the BFKL limit for light-ray operators (3.5) is $\omega=j-1 \rightarrow 0, g^{2} \rightarrow 0$ but $\frac{g^{2}}{\omega} \sim 1$. From eq. (3.10) we see that in this limit only gluon light-rays survive so hereafter we will identify $S_{1}^{\omega}$ from eq. (2.3) with $\mathcal{F}^{\omega}$. It is well known that anomalous dimension of light-ray operators $\mathcal{F}^{\omega}$ in the BFKL limit is given by the solution of equation $\omega=\aleph(\Delta)$ where $\Delta$ is the dimension of the operator and $\aleph(\Delta)$ is the famous Pomeron intercept

$$
\omega=\tilde{\aleph}(\gamma+\omega) \rightarrow \gamma=\gamma^{*}\left(\omega, g^{2}\right)
$$

where $\gamma+\omega=\Delta-3$ and $\tilde{\aleph}(\gamma)$ is the pomeron intercept

$$
\tilde{\aleph}\left(\gamma, g^{2}\right)=4 g^{2}\left[2 \psi(1)-\psi\left(-\frac{\gamma}{2}\right)-\psi\left(1+\frac{\gamma}{2}\right)\right]+O\left(g^{4}\right)
$$

At present, two more terms in the perturbative expansion of the intercept are known [20-22]. 
The coefficient $C(\omega, \Delta)$ in the BFKL limit was calculated in ref. [17] (see also eq. (5.37) below)

$$
C(j, \Delta)=16 g^{2} N_{c}^{2} \frac{2^{1-2 \xi^{*}} \pi}{\xi^{* 2} \sin \pi \xi^{*} \Gamma^{2}\left(1-\frac{\xi^{*}}{2}\right) \Gamma^{2}\left(\frac{1}{2}+\frac{\xi^{*}}{2}\right) \aleph^{\prime}\left(\xi^{*}\right)}
$$

where $\xi^{*}=\gamma^{*}+\omega=\Delta-3$.

As I mentioned in the Introduction, the goal of this paper is to obtain the structure constant $C\left(\Delta_{i}, j_{i}\right)$ in the triple BFKL limit $g^{2} \rightarrow 0, \omega_{1} \sim \omega_{2} \sim \omega_{3} \rightarrow 0$, but $\frac{g^{2}}{\omega} \sim 1$. It will be demonstrated that the structure constant as a function of $g^{2}$ and $\omega_{i}=j_{i}-1$ has the form

$$
\begin{aligned}
& C\left(j_{i}, \Delta_{i}, g^{2}\right) \\
& =\frac{i N_{c}^{2} \omega_{1} \omega_{2} \omega_{3} F\left[\gamma^{*}\left(\omega_{1}, g^{2}\right), \gamma^{*}\left(\omega_{2}, g^{2}\right), \gamma^{*}\left(\omega_{3}, g^{2}\right)\right]}{\pi^{3}\left(\omega_{1}+\omega_{2}-\omega_{3}\right)\left(\omega_{1}+\omega_{3}-\omega_{2}\right)\left(\omega_{2}+\omega_{3}-\omega_{1}\right)}\left[1+O\left(g^{2}\right)+O\left(\omega_{i}\right)+O\left(\frac{1}{N_{c}^{2}}\right)\right]
\end{aligned}
$$

where function $F\left(\gamma_{1}, \gamma_{2}, \gamma_{3}\right)$ is given by a certain integral over two-dimensional coordinates represented as a quartic Mellin-Barnes integral in appendix D. It should be noted that the singularities $\left(\omega_{i}-\omega_{j}-\omega_{k}\right)^{-1}$ are of general nature and come from the boost invariance of the correlator (3.9) in the limit $n_{j} \rightarrow n_{k}$ [26], see the discussion in appendix C.

For the calculation of structure constants I use the method developed in refs. [26] and [27] based on calculation of correlators of "Wilson frames" operators which are basically the light-ray operators with point-splitting UV regularization. It is explained in the next section.

\section{Wilson frames}

As we demonstrated in ref. [17], one cannot study correlators of LR operators in the BFKL approximation since the contribtions would be singular. Instead, one should consider the "Wilson frame" - LR operator with the point splitting in the transverse direction, see e.g. figure 1 for the gluon operator. We need the "forward" Wilson frame integrated over total translation in the corresponding light-like direction

$$
\begin{aligned}
\mathcal{F}_{n}^{j}\left(x_{1 t}, x_{2 t}\right) \equiv & \int_{0}^{\infty} d u u^{1-j} \mathcal{F}_{n}\left(u ; x_{1 t}, x_{2 t}\right), \\
\mathcal{F}_{n}\left(u ; x_{1 t}, x_{2 t}\right) \equiv & \int d v 2 \operatorname{Tr}\left\{F_{n \xi}\left(x_{1 t}+u n+v n\right)\left[u n+v n+x_{1 t}, v n+x_{1 t}\right]\right. \\
& \left.\times\left[v n+x_{1 t}, v n+x_{2 t}\right] F_{n}{ }^{\xi}\left(x_{2 t}+v n\right)\left[x_{2 t}+v n, x_{2 t}+u n+v n\right]\right\} .
\end{aligned}
$$

As $x_{1 t} \rightarrow x_{2 t}$ the Wilson-frame operator $\mathcal{F}_{n}\left(l ; x_{1 t}, x_{2 t}\right)$ reduces to LR operator $\mathcal{F}\left(l, x_{1 t}\right)$ defined in eq. (3.6). Moreover, it is intuitively clear that the point splitting $x_{12_{t}}$ serves as an UV cutoff for the light-ray operator in this limit, at least in the leading log approximation.

One can define also gluino and scalar "Wilson frames" by similar formulas and write down combinations but, as we mentioned above, we do not need their explicit form since at small $\omega$ 's everything is determined by gluon operators $\mathcal{F}^{j}$. Thus, we define Wilson-frame operators (4.1) stretched in $n_{1}, n_{2}$ or $n_{3}$ directions and calculate their correlator at small $\omega_{i}$. 


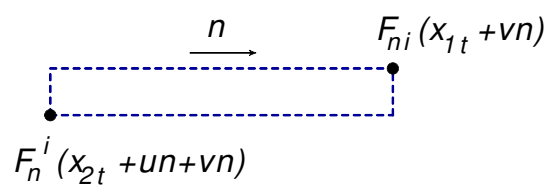

Figure 1. Gluon "Wilson frame" operator.

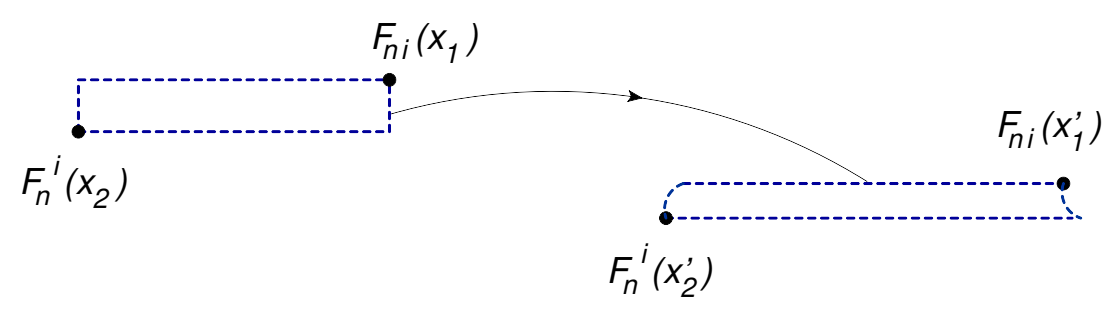

Figure 2. Transformation of Wilson frame under inversion $x_{\mu} \rightarrow x_{\mu} / x^{2}$.

It should be emphasized that narrow Wilson-frame operators are approximately conformally invariant: if one makes the inversion around the point $\left(0,0,0, a_{t}\right)$ one gets the long and narrow Wilson frame with somewhat distorted ends, see figure 2. However, since we are calculating the correlators of Wilson frames in the leading BFKL approximation, the logarithmic integrals are determined by the whole range of integration over $u$ and small corrections at the fringes can be neglected in the leading-log approximation. Thus, one should expect the conformal formulas for the two- and three- point correlators of Wilson frames in the limit of small width of frames of the same form as eqs. (3.8) and (3.9).

$$
\left\langle\mathcal{F}_{n_{1}}^{j}\left(z_{t}+\frac{w_{t}}{2}, z_{t}-\frac{w_{t}}{2}\right) \mathcal{F}_{n_{2}}^{j^{\prime}}\left(z_{t}^{\prime}+\frac{w_{t}^{\prime}}{2}, z_{t}^{\prime}-\frac{w_{t}^{\prime}}{2}\right)\right\rangle \stackrel{w_{t}, w_{t}^{\prime} \rightarrow 0}{=} \delta\left(\nu-\nu^{\prime}\right) \frac{C(\nu, \Delta)}{\left|z_{t}-z_{t}^{\prime}\right|^{2 \Delta(j)}}\left|w_{t} w_{t}^{\prime}\right|^{\gamma(j)}
$$

and

$$
\begin{aligned}
& \left\langle\mathcal{F}_{n_{1}}^{j_{1}}\left(z_{1_{t}}+\frac{w_{1_{t}}}{2}, z_{1_{t}}-\frac{w_{1_{t}}}{2}\right) \mathcal{F}_{n_{2}}^{j_{2}}\left(z_{2_{t}}+\frac{w_{2_{t}}}{2}, z_{2_{t}}-\frac{w_{2_{t}}}{2}\right) \mathcal{F}_{n_{3}}^{j_{3}}\left(z_{3 t}+\frac{w_{3_{t}}}{2}, z_{3_{t}}-\frac{w_{3_{t}}}{2}\right)\right\rangle \\
& \stackrel{w_{i_{t}} \rightarrow 0}{=} C\left(\Delta_{i}, j_{i}\right) \frac{s_{12}{ }^{\frac{j_{1}+j_{2}-j_{3}-1}{2}}\left|w_{1_{t}}\right|^{\gamma\left(j_{1}\right)}}{\left|z_{12_{t}}\right|^{\Delta\left(j_{1}\right)+\Delta\left(j_{2}\right)-\Delta\left(j_{3}\right)-1}} \frac{s_{13}^{\frac{j_{1}+j_{3}-j_{2}-1}{2}}\left|w_{2_{t}}\right|^{\gamma\left(j_{2}\right)}}{\left|z_{13_{t}}\right|^{\Delta\left(j_{1}\right)+\Delta\left(j_{3}\right)-\Delta\left(j_{2}\right)-1}} \frac{\left.s_{23}{ }^{\frac{j_{2}+j_{3}-j_{1}-1}{2}}\left|w_{3_{t}}\right|^{\gamma\left(j_{3}\right)}\right|^{\Delta\left(j_{2}\right)+\Delta\left(j_{3}\right)-\Delta\left(j_{1}\right)-1}}{\left.\mid z_{2}\right)}
\end{aligned}
$$

with point-splitting distances $w_{t}$ serving as UV cutoffs similar to cutoff $\mu$ for the light-ray operators in eqs. (3.8) and (3.9).

Our goal is the three-point formula (4.3) but first I remind the derivation of the BFKL asymptotics of two-point correlator (4.2) obtained in ref. [17] which will serve as a building block for three-frame calculation.

\section{Correlator of two Wilson frames in the BFKL limit}

The CF of two Wilson-frame operators in Regge kinematics is calculated in the same way as four-point correlator of local operators $\left\langle T\left\{\mathcal{O}\left(x_{1}\right) \mathcal{O}\left(x_{2}\right) \mathcal{O}\left(y_{1}\right) \mathcal{O}\left(y_{2}\right)\right\}\right\rangle$ in the Regge limit $x_{1 n_{1}}, y_{1 n_{2}} \rightarrow \infty, x_{2 n_{1}}, y_{2 n_{2}} \rightarrow-\infty$ and the rest of coordinates fixed. (Hereafter I use the notation $x_{n} \equiv x \cdot n$ ). Let me remind the essential steps of such calculation (see e.g. ref. [36]). 


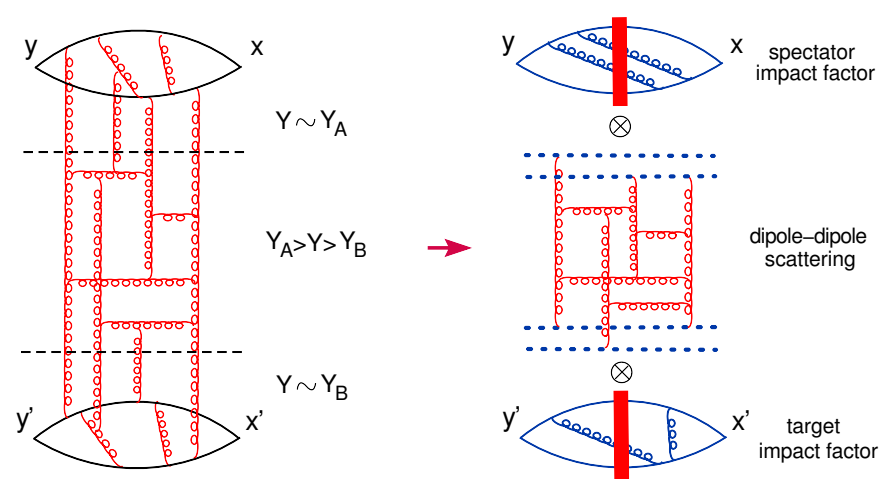

Figure 3. Rapidity factorization for 4-point correlator in the Regge limit.

\subsection{Rapidity factorization for 4-point correlators in the Regge limit}

Let us consider the correlator of four scalar operators ${ }^{4}$

$$
\begin{aligned}
& A\left(x_{1}, x_{2}, x_{3}, x_{4}\right) \equiv \mu^{-4}\left(\mu^{4} x_{12}^{2} x_{34}^{2}\right)^{2+\gamma_{o}}\left\langle T\left\{\mathcal{O}\left(x_{1}\right) \mathcal{O}\left(x_{2}\right) \mathcal{O}\left(x_{3}\right) \mathcal{O}\left(x_{4}\right)\right\}\right\rangle \\
& x_{1}=u_{1} n_{1}+x_{1_{\perp}}, \quad x_{2}=v_{1} n_{1}+x_{2_{\perp}}, \quad x_{3}=u_{2} n_{2}+x_{1_{\perp}}, \quad x_{4}=v_{2} n_{2}+x_{4_{\perp}}
\end{aligned}
$$

where $\gamma_{o}$ is the anomalous dimension of $\mathcal{O}$. In the Regge limit $s_{12}=-2 n_{1} \cdot n_{2} \rightarrow \infty$ and $x_{i_{\perp}}$ fixed. The amplitude (5.1) is a function of two conformal ratios which can be chosen in the Regge limit as

$$
\begin{aligned}
& R=\frac{x_{13}^{2} x_{24}^{2}}{x_{12}^{2} x_{34}^{2}} \simeq \frac{u_{1} u_{2} v_{1} v_{2} s_{12}^{2}}{x_{12_{t}}^{2} x_{34_{t}}^{2}} \\
& r=R\left[1-\frac{x_{14}^{2} x_{23}^{2}}{x_{13}^{2} x_{24}^{2}}+\frac{1}{R}\right]^{2} \simeq \frac{\left(u_{1} u_{2} x_{34_{\perp}}^{2}+v_{1} v_{2} x_{12_{\perp}}^{2}-u_{1} v_{2} x_{23_{\perp}}^{2}-v_{1} u_{2} x_{14_{\perp}}^{2}\right)^{2}}{u_{1} u_{2} v_{1} v_{2} x_{12 t}^{2} x_{34_{\perp}}^{2}}
\end{aligned}
$$

so that $R$ increases with "energy" $s_{12}=-2 n_{1} \cdot n_{2}$ while $r$ is energy-independent. ${ }^{5}$ This corresponds to the momentum-space definition of Regge limit $s / m_{\perp}^{2} \gg 1$ where $m_{\perp}^{2}$ is a characteristic mass scale of the process, in our case the scale of inverse characteristic transverse distances.

In general, the calculation of particle scattering in the Regge limit is based on the rapidity factorization of the amplitude into the product of "projectile impact factor" with rapidities close to those of the projectile particle, "target impact factor" with rapidities close to the those of the target, and scattering of color dipoles encompassing the rapidities in the region between projectile and the target, see figure 3. Technically, one expands the $T\left\{\mathcal{O}\left(x_{1}\right) \mathcal{O}\left(x_{2}\right)\right\}$ in the set of Wilson-line operators with the first being so-called "color dipole" $\mathcal{U}\left(z_{1_{\perp}}, z_{2_{\perp}}\right)$

$$
\begin{aligned}
T\left\{\mathcal{O}\left(x_{1}\right) \mathcal{O}\left(x_{2}\right)\right\} & =\int d^{2} z_{1_{\perp}} d^{2} z_{2_{\perp}} I\left(x_{1}, x_{2} ; z_{1_{\perp}}, z_{2_{\perp}}\right) \operatorname{Tr}\left\{U\left(z_{1_{\perp}}\right) U^{\dagger}\left(z_{2_{\perp}}\right)\right\}+\ldots \\
\mathcal{U}\left(z_{1_{\perp}}, z_{2_{\perp}}\right) & \equiv 1-\frac{1}{N_{c}} \operatorname{Tr}\left\{U\left(z_{1_{\perp}}\right) U^{\dagger}\left(z_{2_{\perp}}\right)\right\}
\end{aligned}
$$

\footnotetext{
${ }^{4}$ For definiteness, one may think about Konishi operator $\mathcal{O}=\phi_{I}^{a} \phi_{I}^{a}$.

${ }^{5}$ To avoid confusion, we reserve the notation $a_{t}$ for the component of the vector $a$ orthogonal to three light-like vectors $n_{1}, n_{2}, n_{3}$ and use the notation $a_{\perp}$ when we discuss components orthogonal to the two light-like vectors $n_{1}$ and $n_{2}$.
} 
where integration goes over $z_{\perp}$ orthogonal to both $n_{1}$ and $n_{2}$, the Wilson line $U$ is defined as

$$
U\left(z_{\perp}\right) \equiv\left[\infty n_{1}+z_{\perp},-\infty n_{1}+z_{\perp}\right]
$$

and dots stand for higher orders of perturbation theory and more Wilson lines. The rapidities $^{6}$ inside the color dipole should be cut from above by characteristic rapidities in the integrals forming the impact factor. To ensure conformal invariance of the rapidity factorization, one should expand in "composite conformal dipoles" introduced in ref. [37].

$$
T\left\{\mathcal{O}\left(x_{1}\right) \mathcal{O}\left(x_{2}\right)\right\}=\int d^{2} z_{1_{\perp}} d^{2} z_{2_{\perp}} I\left(x_{1}, x_{2} ; z_{1_{\perp}}, z_{2}\right) \mathcal{U}_{\text {conf }}^{Y_{A}}\left(z_{1}, z_{2}\right)+\ldots
$$

where

$$
\mathcal{U}_{\text {conf }}^{Y}\left(z_{1}, z_{2}\right)=1-\frac{1}{N_{c}} \operatorname{Tr}\left\{U\left(z_{1_{\perp}}\right) U^{\dagger}\left(z_{2_{\perp}}\right)\right\}+\alpha_{s} \times \text { (four Wilson lines correction) }
$$

is a conformal composite dipole and $Y_{A}=\frac{1}{2} \ln \frac{4 x_{1 n_{2}} x_{2 n_{2}}}{s_{12} x_{12 t}^{2}}$ is the conformally invariant rapidity cutoff. The explicit form of the 4-lines correction is presented in ref. [37], but we do not need it for the leading BFKL logs.

Since we are interested in Regge asymptotics, it is sufficient to consider highest eigenvalue of BFKL intercept with spin 0. Defining a projection of the conformal dipole (5.6) on Lipatov's eigenfunctions [32] with spin 0 , we get

$$
\begin{aligned}
\mathcal{U}_{\text {conf }}^{Y}\left(\nu, z_{0}\right) & \equiv \frac{1}{\pi^{2}} \int \frac{d^{2} z_{1} d^{2} z_{2}}{z_{12}^{4}}\left(\frac{z_{12}^{2}}{z_{10}^{2} z_{20}^{2}}\right)^{\frac{1}{2}-i \nu} \mathcal{U}_{\text {conf }}^{Y}\left(z_{1}, z_{2}\right) \\
\mathcal{U}_{\text {conf }}^{Y}\left(z_{1}, z_{2}\right) & =\int_{-\infty}^{\infty} d \nu \frac{\nu^{2}}{\pi^{2}} \int d^{2} z_{0}\left(\frac{z_{12}^{2}}{z_{10}^{2} z_{20}^{2}}\right)^{\frac{1}{2}+i \nu} \mathcal{U}_{\text {conf }}^{Y}\left(\nu, z_{0}\right)+\text { higher spins }
\end{aligned}
$$

and therefore one can rewrite eq. (5.5) as follows

$$
T\left\{\mathcal{O}\left(x_{1}\right) \mathcal{O}\left(x_{2}\right)\right\}=\int d^{2} z_{0} \int d \nu I_{A}(\nu)\left[\frac{x_{12}^{2} x_{1 n_{2}} x_{2 n_{2}}}{\frac{\left(x_{1}-z_{0}\right)_{\perp}^{2}}{x_{1 n_{2}}}-\frac{\left(x_{2}-z_{0}\right)_{\perp}^{2}}{x_{2 n_{2}}}}\right]^{\frac{1}{2}+i \nu} \mathcal{U}_{\mathrm{conf}}^{Y_{A}}\left(\nu, z_{0}\right) .
$$

Repeating the same expansion for the "target" we get

$$
T\left\{\mathcal{O}\left(x_{3}\right) \mathcal{O}\left(x_{4}\right)\right\}=\int d^{2} z_{0} \int d \nu I_{A}(\nu)\left[\frac{x_{34}^{2} x_{3 n_{1}} x_{4 n_{1}}}{\frac{\left(x_{3}-z_{0}\right)_{\perp}^{2}}{x_{3 n_{1}}}-\frac{\left(x_{4}-z_{0}\right)_{\perp}^{2}}{x_{4 n_{1}}}}\right]^{\frac{1}{2}+i \nu} \mathcal{V}_{\mathrm{conf}}^{Y_{B}}\left(\nu, z_{0}\right),
$$

where $Y_{B}=\frac{1}{2} \ln \frac{4 x_{3 n_{1}} x_{4 n_{1}}}{s_{12} x_{34}^{2}}$ and the conformal dipole $\mathcal{V}_{\text {conf }}^{Y_{B}}\left(\nu, z_{0}\right)$ is defined as

$$
\begin{aligned}
\mathcal{V}_{\text {conf }}^{Y_{B}}\left(\nu, z_{0}\right) & \equiv \frac{1}{\pi^{2}} \int \frac{d^{2} z_{3} d^{2} z_{4}}{z_{34}^{4}}\left(\frac{z_{34}^{2}}{z_{30}^{2} z_{40}^{2}}\right)^{\frac{1}{2}-i \nu} \mathcal{V}_{\mathrm{conf}}^{Y_{B}}\left(z_{3}, z_{4}\right) \\
\mathcal{V}_{\mathrm{conf}}^{Y_{B}}\left(z_{3_{\perp}}, z_{4_{\perp}}\right) & \equiv 1-\frac{1}{N_{c}} \operatorname{Tr}\left\{V\left(z_{3_{\perp}}\right) V^{\dagger}\left(z_{4_{\perp}}\right)\right\}_{\mathrm{conf}}^{Y_{B}}
\end{aligned}
$$

\footnotetext{
${ }^{6}$ The definition of rapidity for the particle with momentum $k=\alpha n_{1}+\beta n_{2}+k_{\perp}$ is $Y \equiv \frac{1}{2} \ln \frac{\alpha}{\beta}$.
} 
where Wilson lines $V$ are ordered along $n_{2}$ direction

$$
V\left(z_{\perp}\right) \equiv\left[\infty n_{2}+z_{\perp},-\infty n_{2}+z_{\perp}\right]
$$

Now the 4-point CF can be represented as an integral of the product of two impact factors $I_{A}(\nu), I_{B}\left(\nu^{\prime}\right)$ and the amplitude of scattering of two color dipoles. In the leading BFKL approximation this amplitude has the form $\left(\alpha_{s} \equiv \frac{g_{\mathrm{YM}}^{2}}{4 \pi}\right)$

$$
\begin{aligned}
& \left\langle\mathcal{U}_{\mathrm{conf}}^{Y_{A}}\left(\nu, z_{0}\right) \mathcal{V}_{\mathrm{conf}}^{Y_{B}}\left(\nu^{\prime}, z_{0}^{\prime}\right)\right\rangle=-\frac{\alpha_{s}^{2}\left(N_{c}^{2}-1\right)}{4 N_{c}^{2}} e^{\aleph\left(\nu, g^{2}\right) \ln Y_{A} Y_{B}} \\
& \quad \times \frac{16 \pi^{2}}{\nu^{2}\left(1+4 \nu^{2}\right)^{2}}\left[\delta\left(z_{0}-z_{0}^{\prime}\right) \delta\left(\nu+\nu^{\prime}\right)+\frac{2^{1-4 i \nu} \delta\left(\nu-\nu^{\prime}\right)}{\pi\left|z_{0}-z_{0}^{\prime}\right|^{2-4 i \nu}} \frac{\Gamma\left(\frac{1}{2}+i \nu\right) \Gamma(1-i \nu)}{\Gamma(i \nu) \Gamma\left(\frac{1}{2}-i \nu\right)}\right] .
\end{aligned}
$$

where

$$
\aleph\left(\nu, g^{2}\right)=4 g^{2}\left[2 \psi(1)-\psi\left(\frac{1}{2}+i \nu\right)-\psi\left(\frac{1}{2}-i \nu\right)\right]+O\left(g^{4}\right)
$$

is the pomeron intercept (3.12).

As I mentioned in the Introduction, in QCD only the $\alpha_{s}^{2}$ correction is known [19] while in $\mathcal{N}=4$ SYM the $g^{6}$ term is known analytically [20-22] and many more can be calculated numerically [20] using Quantum Spectral Curve method [38].

Assembling the result for the 4-point $\mathrm{CF}(5.1)$ one gets the result in the form of general formula [39] for correlators in the "Regge + large $N_{c}$ " limit

$$
A\left(x_{i}\right) \stackrel{s_{12} \rightarrow \infty}{=} \frac{i}{2} \int d \nu f_{+}\left(\aleph\left(g^{2}, \nu\right)\right) F\left(g^{2}, \nu\right) \Omega(r, \nu) R^{\aleph\left(g^{2}, \nu\right) / 2}
$$

where $f_{+}(\aleph)=\frac{e^{i \pi \aleph}-1}{\sin \pi \aleph}$ is a signature factor and

$$
\Omega(r, \nu)=\frac{\nu}{2 \pi^{2}} \frac{\sin 2 \nu \rho}{\sinh \rho}, \quad \cosh \rho=\frac{\sqrt{r}}{2}
$$

is a solution of the Laplace equation in $H_{3}$ hyperboloid $\left(\partial_{H_{3}}^{2}+\nu^{2}+1\right) \Omega(r, \nu)=0$. The dynamics is described by the pomeron intercept $\aleph\left(g^{2}, \nu\right)$ and the "pomeron residue" $F\left(g^{2}, \nu\right)$. The formula (5.14) was proved in [39] (see also [23]) by considering the leading Regge pole in a conformal theory. Also, it was demonstrated up to the NLO level that the structure (5.14) is reproduced by the high-energy OPE in Wilson lines [28, 40, 41].

\subsection{Correlator of two Wilson frames in the Regge limit}

The Regge limit for CF of two Wilson-frame operators means that longitudinal length of frame is much greater than the transverse separation between the frames and the width of frames is even less. As we mentioned, at small frame widths the frames are approximately conformally invariant so one may expect that the general formula (5.14) is applicable. At $x_{12}^{2}, x_{34}^{2} \rightarrow 0$ one gets

$$
r \rightarrow \frac{\left(u_{1}-v_{1}\right)^{2}\left(u_{2}-v_{2}\right)^{2} x_{13_{\perp}}^{4}}{u_{1} v_{1} u_{2} v_{2} x_{12_{\perp}}^{2} x_{34_{\perp}}^{2}}
$$


and

$$
\Omega(r, \nu) \rightarrow \frac{\nu}{2 \pi^{2} i}\left(r^{-\frac{1}{2}+i \nu}-r^{-\frac{1}{2}-i \nu}\right)
$$

Moreover, if we consider "forward" correlation function

$$
\begin{aligned}
& A\left(l, l^{\prime} ; x_{1_{\perp}}, x_{2_{\perp}}, x_{3_{\perp}}, x_{4_{\perp}}\right) \\
& \equiv \int_{0}^{\infty} d v_{1} d v_{2} A\left(l n_{1}+v_{1} n_{1}+x_{1_{\perp}}, v_{1} n_{1}+x_{2_{\perp}}, l^{\prime} n_{2}+v_{2} n_{2}+x_{3_{\perp}}, v_{2} n_{2}+x_{4_{\perp}}\right)
\end{aligned}
$$

the eq. (5.14) reduces to

$$
\begin{aligned}
A\left(l, l^{\prime} ; x_{1_{\perp}}, x_{2_{\perp}}, x_{3_{\perp}}, x_{4_{\perp}}\right) \stackrel{x_{12}^{2}, x_{34}^{2}}{=} & \rightarrow 0 \\
= & l l^{\prime} i \int d \nu \Phi\left(\nu, g^{2}\right) \\
& \times\left(\frac{x_{12_{\perp}}^{2} x_{34 \perp}^{2}}{x_{13_{\perp}}^{4}}\right)^{\frac{1}{2}+i \nu}\left(\frac{l^{2} l^{\prime 2}}{x_{12_{\perp}}^{2} x_{34 \perp}^{2}}\right)^{\aleph\left(\nu, g^{2}\right) / 2} f_{+}(\aleph)
\end{aligned}
$$

As noted in section 4, at small widths Wilson frames are approximately conformally invariant so we need to obtain the representation of eq. (5.19) type for the correlator

$$
\left\langle\mathcal{F}_{n_{1}}\left(l ; z_{t}+\frac{w_{t}}{2}, z_{t}-\frac{w_{t}}{2}\right) \mathcal{F}_{n_{2}}\left(l^{\prime} ; z_{t}^{\prime}+\frac{w_{t}^{\prime}}{2}, z_{t}^{\prime}-\frac{w_{t}^{\prime}}{2}\right)\right\rangle
$$

at $l, l^{\prime} \rightarrow \infty$ (which corresponds to $j \rightarrow 1 \Leftrightarrow \omega \rightarrow 0$ after integration over $l, l^{\prime}$ ). In ref. [17] we performed calculation of CF of two Wilson-frame operators

$$
\left\langle\mathcal{F}_{n_{1}}\left(l ; z_{t}+\frac{w_{t}}{2}, z_{t}-\frac{w_{t}}{2}\right) \mathcal{F}_{n_{2}}\left(l^{\prime} ; z_{t}^{\prime}+\frac{w_{t}^{\prime}}{2}, z_{t}^{\prime}-\frac{w_{t}^{\prime}}{2}\right)\right\rangle
$$

in Regge kinematics in the same way as four-point correlator of local operators. In this section I'll reproduce that calculation in a slightly different way useful for considering 3 -frame correlator in the next section.

We introduce some "rapidity divide" $Y_{0}$ between $Y_{A}$ and $Y_{B}$ and integrate between $Y_{A}$ and $Y_{0}$ and between $Y_{0}$ and $Y_{B}$ in the leading BFKL approximation. After that, we need to convolute the results with the leading order dipole-dipole scattering amplitude.

The first step is the expansion of Wilson frame in color dipoles. The impact factor for Wilson frame, i.e. the coefficient of expansion of "Wilson frame" in color dipoles was calculated in ref. [17]

$$
\begin{aligned}
& \mathcal{F}_{n}\left(l ; x_{\perp}, y_{\perp}\right)=\frac{N_{c}^{2}}{4 \pi^{3} l} \int d^{2} z_{\perp}\left\{1-\mathcal{U}\left(x_{\perp}, z_{\perp}\right)-\mathcal{U}\left(z_{\perp}, y_{\perp}\right)\right. \\
& \left.\quad+\mathcal{U}\left(x_{\perp}, z_{\perp}\right) \mathcal{U}\left(z_{\perp}, y_{\perp}\right)+O\left(\frac{1}{N_{c}}\right)\right\}^{Y_{A}}\left[\frac{2}{(x-z)_{\perp}^{2}(y-z)_{\perp}^{2}}-\frac{4(x-z, y-z)_{\perp}^{2}}{(x-z)_{\perp}^{4}(y-z)_{\perp}^{4}}\right]
\end{aligned}
$$

where the rapidity cutoff is

$$
Y_{A}=\frac{1}{2} \ln \frac{l^{2} s_{12}}{(x-y)_{\perp}^{2}}
$$


by analogy with four-point correlator. ${ }^{7}$ As explained in ref. [17], in order to calculate the correlator of two Wilson frames we need to take into account only the linear terms in eq. (5.22) so we can neglect the last quadratic term. To get the evolution of dipoles in eq. (5.22) from $Y_{A}$ to $Y_{0}$ we project onto Lipatov's eigenfunctions, i.e. rewrite in terms of conformal dipoles and evolve these conformal dipoles in the leading BFKL order.

The projection of eq. (5.22) on Lipatov's eigenfunctions with spin 0 reads

$$
\begin{aligned}
& \mathcal{F}_{n}\left(l ; x_{\perp}, y_{\perp}\right) \\
& =-\frac{N_{c}^{2}}{4 \pi^{3} l} \int d \nu \frac{\nu^{2}}{\pi^{2}} \int d^{2} z_{0} \mathcal{U}_{\mathrm{conf}}^{a}\left(\nu, z_{0}\right) \int d^{2} z_{\perp}\left[\frac{2}{(x-z)_{\perp}^{2}(y-z)_{\perp}^{2}}-\frac{4(x-z, y-z)_{\perp}^{2}}{(x-z)_{\perp}^{4}(y-z)_{\perp}^{4}}\right] \\
& \times\left[\left(\frac{(x-z)_{\perp}^{2}}{\left(x-z_{0}\right)_{\perp}^{2}\left(z-z_{0}\right)_{\perp}^{2}}\right)^{\frac{1}{2}+i \nu}+\left(\frac{(y-z)_{\perp}^{2}}{\left(y-z_{0}\right)_{\perp}^{2}\left(z-z_{0}\right)_{\perp}^{2}}\right)^{\frac{1}{2}+i \nu}\right] \\
& =\frac{N_{c}}{\pi^{2} l} \int d \nu \frac{\nu^{2}}{\pi^{2}} \frac{\frac{1}{2}+i \nu}{\frac{1}{2}-i \nu} \int d^{2} z_{0}\left[\frac{2\left(x-z_{0}, y-z_{0}\right)_{\perp}^{2}}{\left(x-z_{0}\right)_{\perp}^{2}\left(y-z_{0}\right)_{\perp}^{2}}-1\right] \frac{\left[(x-y)_{\perp}^{2}\right]^{-\frac{1}{2}+i \nu} \mathcal{U}_{\mathrm{conf}}\left(\nu, z_{0}\right)^{Y_{A}}}{\left[\left(x-z_{0}\right)_{\perp}^{2}\right]^{\frac{1}{2}+i \nu}\left[\left(y-z_{0}\right)_{\perp}^{2}\right]^{\frac{1}{2}+i \nu}}
\end{aligned}
$$

where we used eq. (4.11) from ref. [17] to get the last line.

Moreover, in the limit of narrow Wilson frame $(x-y)_{\perp} \rightarrow 0$ the integral in the r.h.s. of the above equation can be simplified. Using eqs. (C.4) and (C.6) from ref. [17] one easily obtains

$$
\begin{gathered}
\int d^{2} z_{0}\left[\frac{2\left(x-z_{0}, y-z_{0}\right)_{\perp}^{2}}{\left(x-z_{0}\right)_{\perp}^{2}\left(y-z_{0}\right)_{\perp}^{2}}-1\right] \frac{\left[(x-y)_{\perp}^{2}\right]^{\frac{1}{2}+i \nu}}{\left[\left(x-z_{0}\right)_{\perp}^{2}\right]^{\frac{1}{2}+i \nu}\left[\left(y-z_{0}\right)_{\perp}^{2}\right]^{\frac{1}{2}+i \nu}}\left(\frac{z_{12}^{2}}{z_{10}^{2} z_{20}^{2}}\right)^{\frac{1}{2}-i \nu} \\
\stackrel{x \rightarrow y}{=} \frac{i \pi}{2 \nu} \frac{\left(\frac{1}{2}-i \nu\right)^{2}}{\left(\frac{1}{2}+i \nu\right)^{2}} 2^{4 i \nu} \frac{\Gamma\left(\frac{1}{2}-i \nu\right) \Gamma(i \nu)}{\Gamma\left(\frac{1}{2}+i \nu\right) \Gamma(-i \nu)}\left(\frac{|x-y|^{2} z_{12}^{2}}{\left(x-z_{1}\right)^{2}\left(x-z_{2}\right)^{2}}\right)^{\frac{1}{2}-i \nu} \\
-\frac{i \pi}{2 \nu} 2^{-4 i \nu} \frac{\Gamma\left(\frac{1}{2}+i \nu\right) \Gamma(-i \nu)}{\Gamma\left(\frac{1}{2}-i \nu\right) \Gamma(i \nu)}\left(\frac{|x-y|^{2} z_{12}^{2}}{\left(x-z_{1}\right)^{2}\left(x-z_{2}\right)^{2}}\right)^{\frac{1}{2}+i \nu}
\end{gathered}
$$

Recalling the definition (5.7) of $\mathcal{U}_{\text {conf }}$ and substituting eq. (5.25) in eq. (5.24) one gets

$$
\mathcal{F}_{n}\left(l ; z_{t}+\frac{w_{t}}{2}, z_{t}-\frac{w_{t}}{2}\right)=\frac{i N_{c}^{2}}{\pi^{3} l} \int d \nu \nu \frac{2^{-4 i \nu} \Gamma\left(\frac{3}{2}+i \nu\right) \Gamma(1-i \nu)}{\Gamma\left(\frac{3}{2}-i \nu\right) \Gamma(1+i \nu)}\left(w_{t}^{2}\right)^{-\frac{1}{2}+i \nu} \mathcal{U}_{\mathrm{conf}}^{Y_{A}}\left(z_{t},-\nu\right)
$$

The BFKL evolution of a conformal dipole reads

$$
\mathcal{U}_{\text {conf }}^{Y_{A}}\left(\nu, z_{0}\right)=e^{\left(Y_{A}-Y_{0}\right) \aleph(\nu)} \mathcal{U}_{\text {conf }}^{Y_{0}}\left(\nu, z_{0}\right)
$$

so the result of integration over rapidities in the region $Y_{A}>Y>Y_{0}$ is

$$
\begin{aligned}
& \mathcal{F}_{n_{1}}\left(l ; z_{t}+\frac{w_{t}}{2}, z_{t}-\frac{w_{t}}{2}\right) \\
& =\frac{i N_{c}^{2}}{\pi^{3} l} \int d \nu \nu \frac{2^{-4 i \nu} \Gamma\left(\frac{3}{2}+i \nu\right) \Gamma(1-i \nu)}{\Gamma\left(\frac{3}{2}-i \nu\right) \Gamma(1+i \nu)}\left(w_{t}^{2}\right)^{-\frac{1}{2}+i \nu} e^{\left(Y_{A}-Y_{0}\right) \aleph\left(\nu, g^{2}\right)} \mathcal{U}_{\text {conf }}^{Y_{0}}\left(z_{t},-\nu\right)
\end{aligned}
$$

where $Y_{A}=\ln l+\frac{1}{2} \ln \frac{s_{12}}{w_{t}^{2}}$.

\footnotetext{
${ }^{7}$ Strictly speaking, by analogy with four-point correlator we get $\frac{1}{2} \ln \frac{u(u+l) s_{12}}{(x-y)^{2}}$ with additional intergation over $u$. However, in ref. [17] it was demonstrated that in the leading log approximation this cutoff can be replaced by (5.23).
} 


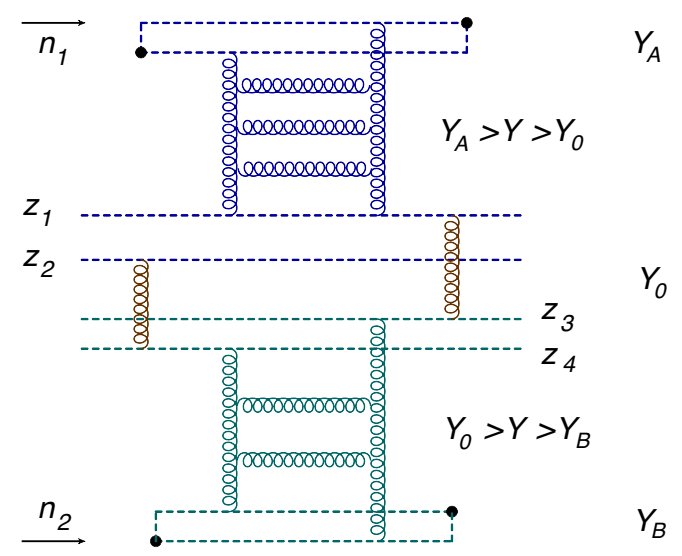

Figure 4. Correlator of two Wilson frames in the Regge limit.

Repeating the same procedure for the bottom part of the diagram in figure 4 one obtains the result of integration over rapidities $Y_{0}>Y$ in the form ${ }^{8}$

$$
\begin{aligned}
& \mathcal{F}_{n_{2}}\left(l^{\prime} ; z_{t}^{\prime}+\frac{w_{t}^{\prime}}{2}, z_{t}^{\prime}-\frac{w_{t}^{\prime}}{2}\right) \\
& =\frac{i N_{c}^{2}}{\pi^{3} l^{\prime}} \int d \nu^{\prime} \nu^{\prime} \frac{2^{-4 i \nu^{\prime}} \Gamma\left(\frac{3}{2}+i \nu^{\prime}\right) \Gamma\left(1-i \nu^{\prime}\right)}{\Gamma\left(\frac{3}{2}-i \nu^{\prime}\right) \Gamma\left(1+i \nu^{\prime}\right)}\left({w^{\prime}}_{t}^{2}\right)^{-\frac{1}{2}+i \nu^{\prime}} e^{\left(Y_{0}+Y_{B}\right) \aleph\left(\nu^{\prime}, g^{2}\right)} \mathcal{V}_{\mathrm{conf}}^{Y_{0}}\left(z_{t}^{\prime},-\nu^{\prime}\right)
\end{aligned}
$$

where $Y_{B}=\ln l^{\prime}+\frac{1}{2} \ln \frac{s_{12}}{w_{t}^{\prime 2}}$.

Using now the result for scattering of color dipoles in the leading perturbative order ${ }^{9}$

$$
\begin{aligned}
& \left\langle\mathcal{U}_{\text {conf }}^{Y_{0}}\left(-\nu, z_{0}\right) \mathcal{V}_{\text {conf }}^{Y_{0}}\left(-\nu^{\prime}, z_{0}^{\prime}\right)\right\rangle \\
& =-\alpha_{s}^{2} \frac{4 \pi^{2}\left(1-\frac{1}{N_{c}^{2}}\right)}{\nu^{2}\left(1+4 \nu^{2}\right)^{2}}\left[\delta\left(z_{0}-z_{0}^{\prime}\right) \delta\left(\nu+\nu^{\prime}\right)-i \nu \frac{2^{1+4 i \nu} \delta\left(\nu-\nu^{\prime}\right)}{\pi\left|z_{0}-z_{0}^{\prime}\right|^{2+4 i \nu}} \frac{\Gamma\left(\frac{1}{2}-i \nu\right) \Gamma(1+i \nu)}{\Gamma(1-i \nu) \Gamma\left(\frac{1}{2}+i \nu\right)}\right] .
\end{aligned}
$$

we get the result for correlator of two Wilson frames in the form of eq. (5.19) type

$$
\begin{aligned}
& \left\langle\mathcal{F}_{n_{1}}\left(l ; z_{t}+\frac{w_{t}}{2}, z_{t}-\frac{w_{t}}{2}\right) \mathcal{F}_{n_{2}}\left(l^{\prime} ; z_{t}^{\prime}+\frac{w_{t}^{\prime}}{2}, z_{t}^{\prime}-\frac{w_{t}^{\prime}}{2}\right)\right\rangle \\
& =-i \frac{g^{2} N_{c}^{2}}{\pi^{3} l l^{\prime}} \int d \nu \frac{2^{3-4 i \nu} \nu}{\left(\frac{1}{2}+i \nu\right)\left(\frac{1}{2}-i \nu\right)^{3}} \frac{\Gamma\left(\frac{3}{2}+i \nu\right) \Gamma(1-i \nu)}{\Gamma\left(\frac{3}{2}-i \nu\right) \Gamma(1+i \nu)} \frac{\left(w_{t}^{2}{w^{\prime}}_{t}^{\prime 2}\right)^{-\frac{1}{2}+i \nu} e^{\left(Y_{A}+Y_{B}\right) \aleph\left(\nu, g^{2}\right)}}{\left[\left(z_{t}-z_{t}^{\prime}\right)^{2}\right]^{1+2 i \nu}} \\
& =-i \frac{g^{2} N_{c}^{2}}{\pi^{3} l l^{\prime}} \int d \nu \frac{2^{3-4 i \nu} \nu\left(l l^{\prime} s_{12}\right)^{\aleph(\nu)}}{\left(\frac{1}{2}+i \nu\right)\left(\frac{1}{2}-i \nu\right)^{3}} \frac{\Gamma\left(\frac{3}{2}+i \nu\right) \Gamma(1-i \nu)}{\Gamma\left(\frac{3}{2}-i \nu\right) \Gamma(1+i \nu)} \frac{\left(w_{t}^{2} w_{t}^{\prime 2}\right)^{-\frac{1}{2}+i \nu-\frac{1}{2} \aleph\left(\nu, g^{2}\right)}}{\left[\left(z_{t}-z_{t}^{\prime}\right)^{2}\right]^{1+2 i \nu}}
\end{aligned}
$$

${ }^{8}$ The difference in signs of $Y_{A}$ and $Y_{B}$ in eqs. (5.28) and (5.29) is due to the fact that replacement $n_{1} \leftrightarrow n_{2}$ should be accompanied by changing the sigh of the rapidity: $\ln \frac{\beta}{\alpha}=-\ln \frac{\alpha}{\beta}$.

${ }^{9}$ As usual, we stop the evolution of color dipoles from upper and lower parts of the diagram in figure 4 at the points $Y_{0}+\delta$ and $Y_{0}-\delta$. The small $\delta$ is such that the relative energy $s_{\delta}=m_{\perp}^{2} e^{2 \delta}$ is greater than the characteristic transverse scale $m_{\perp}^{2}$ but $g^{2} \ln \frac{s_{\delta}}{m^{2}}=2 g^{2} \delta \ll 1$. In this case, one does not need to include evolution between $Y_{0}+\delta$ and $Y_{0}-\delta$ but can still use the three-level formula

$$
\left\langle\mathcal{U}\left(z_{1_{\perp}}, z_{2_{\perp}}\right) \mathcal{V}\left(z_{3_{\perp}}, z_{4_{\perp}}\right)\right\rangle=-\left(1-\frac{1}{N_{c}^{2}}\right) \frac{\alpha_{s}^{2}}{8} \ln ^{2} \frac{z_{13_{\perp}}^{2} z_{24_{\perp}}^{2}}{z_{14_{\perp}}^{2} z_{23_{\perp}}^{2}}
$$

which translates to eq. (5.30) after projection on spin-0 eigenfunctions. 
Note that the "rapidity divide" $Y_{0}$ disappeared from the result. Moreover, the scattering amplitude (5.31) depends only on product of $l$ and $l^{\prime}$ which is a reflection of boost invariance of the original amplitude (4.1): it is easy to see that if one makes boost $n_{1} \rightarrow \lambda n_{1}$ and $n_{2} \rightarrow \frac{1}{\lambda} n_{2}$ the correlator (4.1) does not change. Now we shall see that this property leads to the $\delta$-function in the correlator (4.2).

Indeed, the integral over $l$ and $l^{\prime}$ have the form

$$
\begin{aligned}
& \left\langle\mathcal{F}_{n_{1}}^{j}\left(z_{t}+\frac{w_{t}}{2}, z_{t}-\frac{w_{t}}{2}\right) \mathcal{F}_{n_{2}}^{j^{\prime}}\left(z_{t}^{\prime}+\frac{w_{t}^{\prime}}{2}, z_{t}^{\prime}-\frac{w_{t}^{\prime}}{2}\right)\right\rangle=-i \frac{g^{2} N_{c}^{2}}{\pi^{3}} \int_{0}^{\infty} d l d l^{\prime} l^{-j} l^{\prime-j^{\prime}} \\
& \times \theta\left(l l^{\prime}-\frac{\left(z_{t}-z_{t}^{\prime}\right)^{2}}{s_{12}}\right) \int d \nu \frac{\nu 2^{3-4 i \nu}\left(l l^{\prime} s_{12}\right)^{\aleph\left(\nu, g^{2}\right)}}{\left(\frac{1}{2}+i \nu\right)\left(\frac{1}{2}-i \nu\right)^{3}} \frac{\Gamma\left(\frac{3}{2}+i \nu\right) \Gamma(1-i \nu)}{\Gamma\left(\frac{3}{2}-i \nu\right) \Gamma(1+i \nu)} \frac{\left(w_{t}^{2} w_{t}^{\prime 2}\right)^{-\frac{1}{2}+i \nu-\frac{1}{2} \aleph\left(\nu, g^{2}\right)}}{\left[\left(z_{t}-z_{t}^{\prime}\right)^{2}\right]^{1+2 i \nu}}
\end{aligned}
$$

where the factor $\theta\left(l l^{\prime}-\frac{\left(z_{t}-z_{t}^{\prime}\right)^{2}}{s_{12}}\right)$ comes from the restriction that the longitudinal size of two Wilson frames should be greater than the relative transverse separation. ${ }^{10}$

Performing the integration over $l$ and $l^{\prime}$ one obtains where $j=\frac{1}{2}+i \varsigma$ and $j^{\prime}=\frac{1}{2}+i \varsigma^{\prime}$.

$$
\begin{aligned}
& \left\langle\mathcal{F}_{n_{1}}^{j}\left(z_{t}+\frac{w_{t}}{2}, z_{t}-\frac{w_{t}}{2}\right) \mathcal{F}_{n_{2}}^{j^{\prime}}\left(z_{t}^{\prime}+\frac{w_{t}^{\prime}}{2}, z_{t}^{\prime}-\frac{w_{t}^{\prime}}{2}\right)\right\rangle=-i \delta\left(\varsigma-\varsigma^{\prime}\right) \frac{g^{2} N_{c}^{2}}{\pi^{2}} \int_{-\infty}^{\infty} d \nu \frac{1}{\omega-\aleph\left(\nu, g^{2}\right)} \\
& \times \frac{\nu 2^{4-4 i \nu} s_{12}^{\omega}}{\left(\frac{1}{2}+i \nu\right)\left(\frac{1}{2}-i \nu\right)^{3}} \frac{\Gamma\left(\frac{3}{2}+i \nu\right) \Gamma(1-i \nu)}{\Gamma\left(\frac{3}{2}-i \nu\right) \Gamma(1+i \nu)} \frac{\left(w_{t}^{2} w_{t}^{\prime 2}\right)^{-\frac{1}{2}+i \nu-\frac{1}{2} \aleph\left(\nu, g^{2}\right)}}{\left[\left(z_{t}-z_{t}^{\prime}\right)^{2}\right]^{j+2 i \nu-\aleph\left(\nu, g^{2}\right)}}
\end{aligned}
$$

Next, we analytically continue this formula to small $\omega=j-1$. To estimate this integral at small $\omega$ 's it is convenient to rewrite it in the variable $\xi=2 i \nu-1$.

$$
\begin{gathered}
\left\langle\mathcal{F}_{n_{1}}^{j}\left(z_{t}+\frac{w_{t}}{2}, z_{t}-\frac{w_{t}}{2}\right) \mathcal{F}_{n_{2}}^{j^{\prime}}\left(z_{t}^{\prime}+\frac{w_{t}^{\prime}}{2}, z_{t}^{\prime}-\frac{w_{t}^{\prime}}{2}\right)\right\rangle=\delta\left(\varsigma-\varsigma^{\prime}\right) g^{4} N_{c}^{2} \int_{-1-i \infty}^{-1+i \infty} \frac{d \xi}{2 \pi i} \frac{1}{\omega-\tilde{\aleph}\left(\xi, g^{2}\right)} \\
\times \frac{2^{5-2 \xi} \pi s_{12}^{\omega}}{\xi^{2} \sin \pi \xi \Gamma^{2}\left(1-\frac{\xi}{2}\right) \Gamma^{2}\left(\frac{1}{2}+\frac{\xi}{2}\right)} \frac{\left(w_{t}^{2} w_{t}^{\prime 2}\right)^{\frac{\xi-\tilde{\aleph}\left(\xi, g^{2}\right)}{2}}}{\left[\left(z_{t}-z_{t}^{\prime}\right)^{2}\right]^{2+\omega+\xi-\tilde{\aleph}\left(\xi, g^{2}\right)}}
\end{gathered}
$$

The notation here is

$$
\tilde{\aleph}\left(\xi, g^{2}\right) \equiv \aleph\left(-i \frac{1+\xi}{2}, g^{2}\right)=4 g^{2}\left[2 \psi(1)-\psi\left(-\frac{\xi}{2}\right)-\psi\left(1+\frac{\xi}{2}\right)\right]+O\left(g^{4}\right)
$$

and we often omit the $g^{2}$ dependence to avoid cluttering of the formulas.

At small $w_{t}^{2}, w_{t}^{\prime 2}$ we can close the contour of $\xi$ integration on the residues in the right half-plane. The two leading poles are located at $\xi^{*}=\tilde{\aleph}^{-1}(\omega)$ and $\xi=0$. Let us consider them in turn. Taking residue at $\xi^{*}=\tilde{\aleph}^{-1}\left(\omega, g^{2}\right)$ we get

$$
\begin{aligned}
& \left\langle\mathcal{F}_{n_{1}}^{j}\left(z_{t}+\frac{w_{t}}{2}, z_{t}-\frac{w_{t}}{2}\right) \mathcal{F}_{n_{2}}^{j^{\prime}}\left(z_{t}^{\prime}+\frac{w_{t}^{\prime}}{2}, z_{t}^{\prime}-\frac{w_{t}^{\prime}}{2}\right)\right\rangle \\
& =\delta\left(\varsigma-\varsigma^{\prime}\right) g^{2} N_{c}^{2} \frac{2^{5-2 \xi^{*}} \pi s_{12}^{\omega}}{\xi^{* 2} \sin \pi \xi^{*} \Gamma^{2}\left(1-\frac{\xi^{*}}{2}\right) \Gamma^{2}\left(\frac{1}{2}+\frac{\xi^{*}}{2}\right) \aleph^{\prime}\left(\xi^{*}\right)} \frac{\left(w_{t}^{2}{w^{\prime}}_{t}^{2}\right)^{\frac{\xi^{*}-\omega}{2}}}{\left[\left(z_{t}-z_{t}^{\prime}\right)^{2}\right]^{2+\xi^{*}}}
\end{aligned}
$$

\footnotetext{
${ }^{10}$ This the $s \gg m_{\perp}^{2}$ requirement for applicability of BFKL approximation recast in the coordinate-space language, see the discussion in ref. [17].
} 
Comparing this equation to general form of two-point correlator of light-ray operators (3.8) we see that $\xi^{*}-\omega$ can be identified with anomalous dimension $\gamma$ so we finally get [17]

$$
\begin{aligned}
& \left\langle\mathcal{F}_{n_{1}}^{j}\left(z_{t}+\frac{w_{t}}{2}, z_{t}-\frac{w_{t}}{2}\right) \mathcal{F}_{n_{2}}^{j^{\prime}}\left(z_{t}^{\prime}+\frac{w_{t}^{\prime}}{2}, z_{t}^{\prime}-\frac{w_{t}^{\prime}}{2}\right)\right\rangle \\
& =\delta\left(\varsigma-\varsigma^{\prime}\right) g^{2} N_{c}^{2} \frac{2^{5-2 \xi^{*}} \pi s_{12}^{\omega}}{\xi^{* 2} \sin \pi \xi^{*} \Gamma^{2}\left(1-\frac{\xi^{*}}{2}\right) \Gamma^{2}\left(\frac{1}{2}+\frac{\xi^{*}}{2}\right) \aleph^{\prime}\left(\xi^{*}\right)} \frac{\left(w_{t}^{2} w_{t}^{\prime 2}\right)^{\frac{\gamma^{*}}{2}}}{\left[\left(z_{t}-z_{t}^{\prime}\right)^{2}\right]^{2+\omega+\gamma^{*}}}
\end{aligned}
$$

where $\gamma^{*}$ is a solution of the equation (3.11) and $\xi^{*}=\gamma^{*}+\omega=\Delta-3$.

Note that this formula is actually at the NLO level: in the leading log approximation we just get $\omega=\aleph\left(\gamma^{*}\right)$ and $\left[\left(z-z^{\prime}\right)_{t}^{2}\right]^{-2-\gamma_{*}}$ in the r.h.s. of eq. (5.37). The reason that we got the NLO equation (3.11) is that we used $Y_{A}=\ln l+\frac{1}{2} \ln s_{12} m_{\perp}^{2}-\frac{1}{2} \ln m_{\perp}^{2} w_{t}^{2}$ where the last term exceeds the LLA accuracy. As demonstrated in ref. [17], we can do this using the exact formula for the 4-point correlator (5.14). Unfortunately, for the 6-point correlator there is no such formula so we cannot promote our LO BFKL calculation to the NLO level.

At $g^{2} \ll \omega \ll 1$ we get $\xi_{*} \simeq-8 \frac{g^{2}}{\omega}$ (recall that $\aleph(\xi) \simeq-8 \frac{g^{2}}{\xi}$ at small $\xi$ ) and therefore the result (5.37) takes the form

$$
\begin{aligned}
& \left\langle\mathcal{F}_{n_{1}}^{j}\left(z_{t}+\frac{w_{t}}{2}, z_{t}-\frac{w_{t}}{2}\right) \mathcal{F}_{n_{2}}^{j^{\prime}}\left(z_{t}^{\prime}+\frac{w_{t}^{\prime}}{2}, z_{t}^{\prime}-\frac{w_{t}^{\prime}}{2}\right)\right\rangle \\
& \simeq-\delta\left(\omega-\omega^{\prime}\right) \frac{N_{c}^{2} \omega s_{12}^{\omega}}{2 \pi\left[\left(z_{t}-z_{t}^{\prime}\right)^{2}\right]^{2+\omega}}\left(\frac{\left(z_{t}-z_{t}^{\prime}\right)^{2}}{\left|w_{t} w_{t}^{\prime}\right|}\right)^{\frac{8 g^{2}}{\omega}+\omega}
\end{aligned}
$$

which agrees with eq. (4.17) from ref. [17].

Let us consider now the pole at $\xi=0$. At small $\xi$

$$
\frac{1}{\omega-\tilde{\aleph}(\xi)} \simeq \frac{\xi}{8 g^{2}}\left(1-\frac{\xi \omega}{8 g^{2}}+\ldots\right)
$$

so the residue at $\xi=0$ yields

$$
\delta\left(\omega-\omega^{\prime}\right) \frac{\omega s_{12}^{\omega} N_{c}^{2}}{2 \pi\left[\left(z_{t}-z_{t}^{\prime}\right)^{2}\right]^{2+\omega}}\left[1+\frac{8 g^{2}}{\omega} \ln \frac{\left(z_{t}-z_{t}^{\prime}\right)^{2}}{\left|w_{t} w_{t}^{\prime}\right|}\right]
$$

Thus, the result for diagrams in figure 4 is a sum of eq. (5.37) and eq. (5.40). However, there are two low-order diagrams shown in figure 5 that are not included in this result since the formula (5.22) is correct starting from the second order of perturbation theory. These diagrams should cancel the contribution of the $\xi=0$ pole (5.40) so the final result (5.37) has proper conformal behavior. The tree-level diagram in figure 5a is calculated in the appendix A and the result (B.3) is minus the first term in the square brackets in eq. (5.40). Similarly, the contribution of diagrams in figure $5 \mathrm{~b}$ should cancel the second term so the contribution of all diagrams (in figure 5 and figure 4 ) is given by eq. (5.37). 


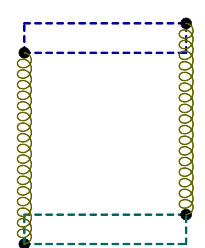

(a)

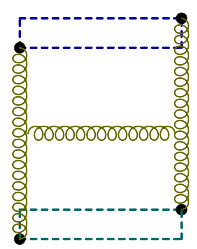

+ perm.

(b)

Figure 5. First (a) and second (b) order diagrams for the correlator of two Wilson frames.

\section{Correlator of three Wilson frames in the triple Regge limit}

\subsection{Triple BFKL evolution}

To get the structure constant in eq. (3.9) at $\omega_{i} \rightarrow 0$ we will consider the correlator of three gluon Wilson frames aligned along $n_{1}, n_{2}$, and $n_{3}$ directions:

$$
\left\langle\mathcal{F}_{n_{1}}^{j_{1}}\left(z_{1_{t}}+\frac{w_{1_{t}}}{2}, z_{1_{t}}-\frac{w_{1_{t}}}{2}\right) \mathcal{F}_{n_{2}}^{j_{2}}\left(z_{2_{t}}+\frac{w_{2_{t}}}{2}, z_{2_{t}}-\frac{w_{2_{t}}}{2}\right) \mathcal{F}_{n_{3}}^{j_{3}}\left(z_{3_{t}}+\frac{w_{3_{t}}}{2}, z_{3_{t}}-\frac{w_{3_{t}}}{2}\right)\right\rangle
$$

A typical diagram is shown in figure 6 .

As usual, we assume that longitudinal lengths of frames are much greater than the transverse separations between the frames and those separations are much greater than widths of the frames. The form of the three-point correlators of light-ray operators (3.9) suggests that this correlator is determined by three BFKL evolutions. It will be demonstrated in this section.

The method to obtain BFKL asymptotics of a scattering amplitude by evolution of Wilson lines is the following. In a typical amplitude like shown in figure $7 \mathrm{a}$ we separate the (gluon) fields according to their rapidity, using the fact that particles with different rapidities perceive each other as Wilson lines, and study the evolution of these Wilson lines with respect to rapidity cutoff. Since we have now three light-like directions, it is convenient to introduce "triple Sudakov variables"

$$
k=\alpha n_{1}+\beta n_{2}+\gamma n_{3}+k_{t}, \quad d^{4} k=\frac{\sqrt{s_{12} s_{13} s_{23}}}{2} d \alpha d \beta d \gamma d k_{t}
$$

and consider factorization in all three of them. ${ }^{11}$

Similarly to the analysis of amplitudes in the usual Regge regime we assume that all $k_{t}^{2} \sim m_{\perp}^{2}$ where $m_{\perp}^{2}$ is of order of (inverse) transverse separations between Wilson frames. Also, we assume that all $s_{i j}$ are of the same order of magnitude $s \gg m_{\perp}^{2}$.

The key observation is that as long as there is a sufficient rapidity space for the evolution of each of Wilson frames these evolutions are the same as for the two-point correlator of Wilson lines. To demonstrate this, consider the evolution of $n_{1}$-parallel Wilson frame schematically depicted by the upper gluon ladder in figure $7 \mathrm{~b}$. It is convenient to

\footnotetext{
${ }^{11}$ As defined in section $2, n_{i}$ are light-like vectors with $s_{i j}=-2 n_{i} \cdot n_{j}$ and $k_{t}$ is orthogonal to all three of them.
} 


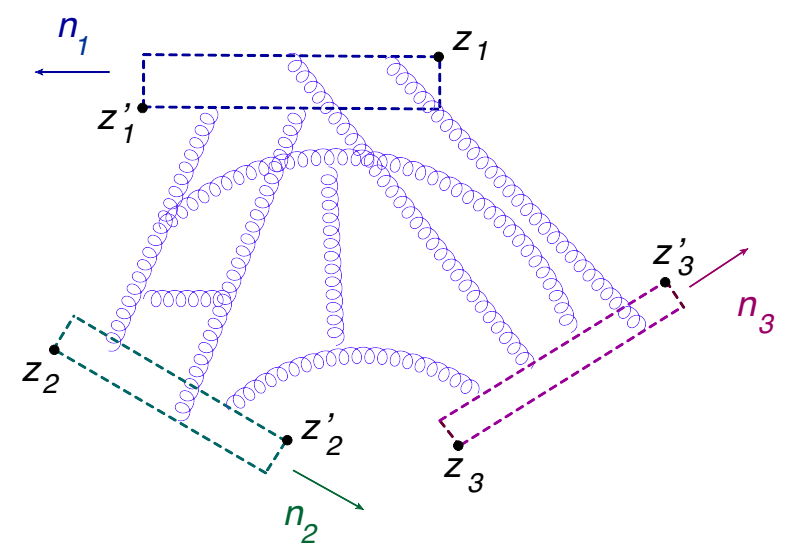

Figure 6. Correlator of three Wison frames.

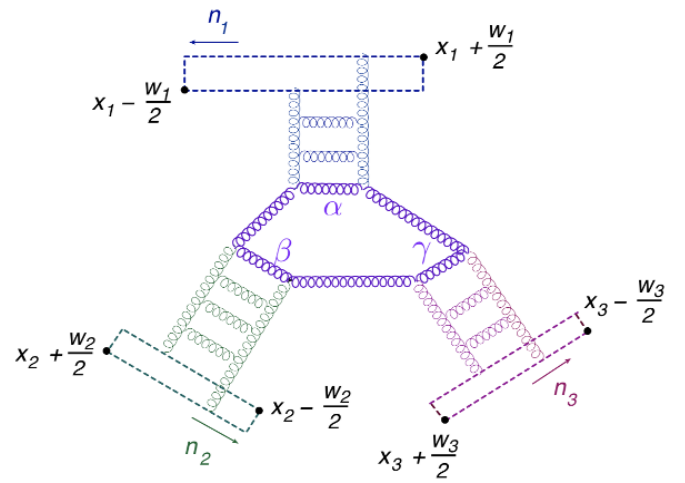

(a)

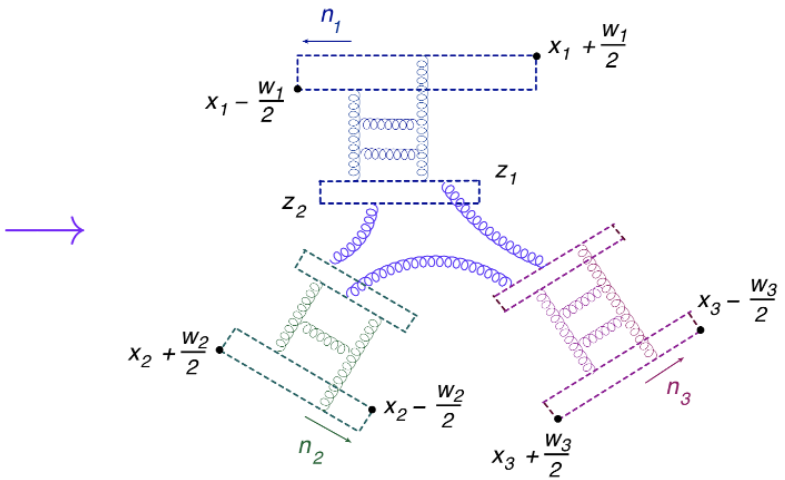

(b)

Figure 7. Triple BFKL evolution.

relate "triple Sudakov" variables (6.2) to usual Sudakov variables

$$
k=\tilde{\alpha} n_{1}+\tilde{\beta} \tilde{n}_{1}+k_{\perp}
$$

where we chose the second light-like vector as

$$
\tilde{n}_{1}=\frac{s_{13}}{2 s_{23}} n_{2}+\frac{s_{12}}{2 s_{23}} n_{3}-\frac{n_{1}}{4}
$$

so that $-2 n_{1} \cdot \tilde{n}_{1} \equiv \tilde{s}=\frac{s_{12} s_{13}}{s_{23}}$. We can rewrite eq. (6.3) as follows

$$
k=\tilde{\alpha} n_{1}+\tilde{\beta} \tilde{n}_{2}+\tilde{k} \tilde{e}+k_{t} e_{t}
$$

where

$$
\tilde{e} \equiv\left(n_{2} \sqrt{\frac{s_{13}}{s_{12} s_{23}}}-n_{3} \sqrt{\frac{s_{12}}{s_{13} s_{23}}}\right), \quad \tilde{e}^{2}=1
$$

The relation between variables (6.2) and (6.3) is

$$
\tilde{\alpha}=\alpha+\frac{\tilde{\beta}}{4}, \quad \tilde{\beta}=\beta \frac{s_{23}}{s_{13}}+\gamma \frac{s_{23}}{s_{12}}, \quad \tilde{k}=\frac{1}{2} \sqrt{\frac{s_{23}}{s_{12} s_{13}}}\left(\beta s_{12}-\gamma s_{13}\right)
$$


As we will demonstrate below, characteristic $\tilde{k}$ are of order of $m_{\perp}$ (see eq. (6.31)) so we can define

$$
k_{\perp}^{2} \equiv k_{t}^{2}+\tilde{k}^{2} \sim m_{\perp}^{2}
$$

In terms of these variables

$$
k^{2}=k_{\perp}^{2}-\tilde{\alpha} \tilde{\beta} \tilde{s}
$$

and the evolution of the $n_{1}$-parallel Wilson lines looks like the evolution considered in section 5.2 for the two-point correlator of Wilson frames. Thus, we can recycle the result (5.28)

$$
\begin{aligned}
& \mathcal{F}_{n_{1}}\left(l_{1} ; x_{1 t}+\frac{w_{1 t}}{2}, x_{1 t}-\frac{w_{1 t}}{2}\right) \\
& =\frac{i N_{c}^{2}}{\pi^{3} l_{1}} \int d \nu_{1} \nu_{1} \frac{2^{-4 i \nu_{1}} \Gamma\left(\frac{3}{2}+i \nu_{1}\right) \Gamma\left(1-i \nu_{1}\right)}{\Gamma\left(\frac{3}{2}-i \nu_{1}\right) \Gamma\left(1+i \nu_{1}\right)}\left(w_{1 t}^{2}\right)^{-\frac{1}{2}+i \nu_{1}} e^{\left(Y_{1}-Y_{1}^{\prime}\right) \aleph\left(\nu_{1}\right)} \mathcal{U}_{\mathrm{conf}}^{Y_{1}^{\prime}}\left(x_{1 t},-\nu_{1}\right)
\end{aligned}
$$

where in the LLA $Y_{1}=\frac{1}{2} \ln \frac{l_{1}^{2} \tilde{s}}{w_{1 t}^{2}} \simeq \ln l_{1}+\frac{1}{2} \ln \tilde{s} m_{\perp}^{2}$ and $Y_{1}^{\prime}$ is the rapidity $\left(\frac{1}{2} \ln \frac{\tilde{\alpha}}{\tilde{\beta}}\right)$ at which we stop the evolution.

To strengthen these coordinate-space arguments in favor of BFKL evolution in the triple Regge limit, it is shown in the appendix A that the standard momentum-space calculation of one-loop diagrams in the triple Regge limit reproduces the first rung of the BFKL ladder for color dipoles.

It should be noted that the arguments in favor of BFKL evolution in the triple Regge limit presented above are somewhat general, so in the appendix A I confirm them by a standard momentum-space calculation of one-loop diagrams in the triple Regge limit which reproduces the first rung of the BFKL ladder for color dipoles.

The explicit form of the conformal dipole (5.7) in the coordinates $z_{t}$ and $\tilde{z}$ reads $^{12}$

$$
\begin{aligned}
\mathcal{U}_{\text {conf }}^{a}\left(\nu_{1}, x_{1 t}\right) \equiv & \frac{1}{\pi^{2}} \int \frac{d z_{1 t} d \tilde{z}_{1} d z_{2 t} d \tilde{z}_{2}}{\left(z_{12 t}^{2}+\tilde{z}_{12}^{2}\right)^{2}} \\
& \times\left(\frac{z_{12 t}^{2}+\tilde{z}_{12}^{2}}{\left[\left(x_{1}-z_{1}\right)_{t}^{2}+\tilde{z}_{1}^{2}\right]\left[\left(x_{1}-z_{2}\right)_{t}^{2}+\tilde{z}_{2}^{2}\right]}\right)^{\frac{1}{2}-i \nu_{1}} \mathcal{U}^{a}\left(z_{1 t} e_{t}+\tilde{z}_{1} \tilde{e}, z_{2 t} e_{t}+\tilde{z}_{2} \tilde{e}\right)
\end{aligned}
$$

Repeating the same procedure for the frame parallel to $n_{2}$ we get

$$
\begin{aligned}
& \mathcal{F}_{n_{2}}\left(l_{2} ; x_{2 t}+\frac{w_{2 t}}{2}, x_{2 t}-\frac{w_{2 t}}{2}\right) \\
& =\frac{i N_{c}^{2}}{\pi^{3} l_{2}} \int d \nu_{2} \nu_{2} \frac{2^{-4 i \nu_{2}} \Gamma\left(\frac{3}{2}+i \nu_{2}\right) \Gamma\left(1-i \nu_{2}\right)}{\Gamma\left(\frac{3}{2}-i \nu_{2}\right) \Gamma\left(1+i \nu_{2}\right)}\left(w_{2 t}^{2}\right)^{-\frac{1}{2}+i \nu_{2}} e^{\left(Y_{2}-Y_{2}^{\prime}\right) \aleph\left(\nu_{2}\right)} \mathcal{V}_{\text {conf }}^{Y_{2}^{\prime}}\left(x_{2 t},-\nu_{2}\right)
\end{aligned}
$$

Here $Y_{2} \simeq \ln l_{2}+\frac{1}{2} \ln \breve{s} m_{\perp}^{2}, Y_{2}^{\prime}$ is the rapidity at which we stop the evolution and

$$
\begin{aligned}
\mathcal{V}_{\text {conf }}^{a}\left(\nu_{2}, x_{2 t}\right) \equiv & \frac{1}{\pi^{2}} \int \frac{d z_{3 t} d \breve{z}_{3} d z_{4 t} d \breve{z}_{4}}{\left(z_{34 t}^{2}+\breve{z}_{34}^{2}\right)^{2}} \\
& \times\left(\frac{z_{34 t}^{2}+\breve{z}_{34}^{2}}{\left[\left(x_{2}-z_{3}\right)_{t}^{2}+\breve{z}_{3}^{2}\right]\left[\left(x_{2}-z_{4}\right)_{t}^{2}+\breve{z}_{4}^{2}\right]}\right)^{\frac{1}{2}-i \nu_{2}} \mathcal{V}^{a}\left(z_{3 t} e_{t}+\breve{z}_{3}, z_{4 t} e_{t}+\breve{z}_{4}\right)
\end{aligned}
$$

\footnotetext{
${ }^{12}$ As mentioned above, in the LLA $\mathcal{U}_{\text {conf }}\left(z_{1}, z_{2}\right)$ can be replaced by $\mathcal{U}\left(z_{1}, z_{2}\right)$.
} 


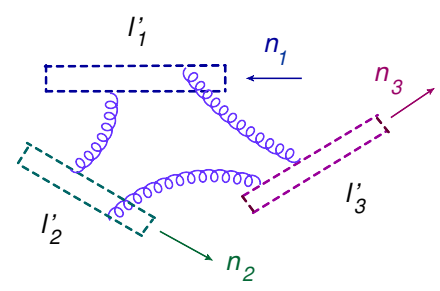

(a)

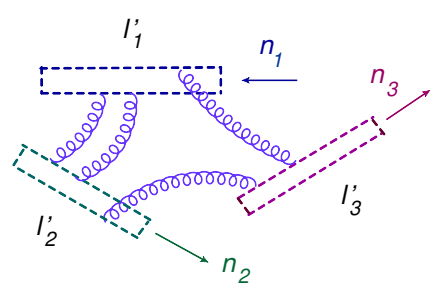

(b)

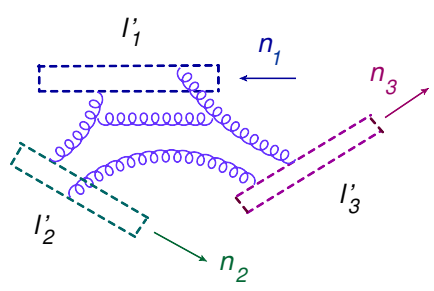

(c)

Figure 8. Three Wilson frames in the leading order ("a") and at the one-loop level ("b"+ "c"+permutations).

where $\mathcal{V}$ is the conformal dipole with Wilson lines parallel to $n_{2}$ and

$$
\breve{e} \equiv\left(n_{3} \sqrt{\frac{s_{12}}{s_{13} s_{23}}}-n_{1} \sqrt{\frac{s_{23}}{s_{12} s_{13}}}\right), \quad \breve{s} \equiv \frac{s_{12} s_{23}}{s_{13}}
$$

Note that the transverse plane $e_{t}, \breve{e}$ for the evolution of second frame is different from the transverse plane $e_{t}, \tilde{e}$ for the first frame.

Similarly one can get the result for the evolution of the third frame in the form

$$
\begin{aligned}
& \mathcal{F}_{n_{3}}\left(l_{3} ; x_{3 t}+\frac{w_{3 t}}{2}, x_{3 t}-\frac{w_{3 t}}{2}\right) \\
& =\frac{i N_{c}^{2}}{\pi^{3} l_{3}} \int d \nu_{3} \nu_{3} \frac{2^{-4 i \nu_{3}} \Gamma\left(\frac{3}{2}+i \nu_{3}\right) \Gamma\left(1-i \nu_{3}\right)}{\Gamma\left(\frac{3}{2}-i \nu_{3}\right) \Gamma\left(1+i \nu_{3}\right)}\left(w_{3 t}^{2}\right)^{-\frac{1}{2}+i \nu_{3}} e^{\left(Y_{3}-Y_{3}^{\prime}\right) \aleph\left(\nu_{3}\right)} \mathcal{W}_{\mathrm{conf}}^{Y_{3}^{\prime}}\left(x_{3 t},-\nu_{3}\right)
\end{aligned}
$$

Here $Y_{3} \simeq \ln l_{3}+\frac{1}{2} \ln \check{s} m_{\perp}^{2}, Y_{3}^{\prime}$ is the rapidity at which we stop the evolution and

$$
\begin{aligned}
\mathcal{W}_{\text {conf }}^{a}\left(\nu_{3}, x_{3 t}\right) \equiv & \frac{1}{\pi^{2}} \int \frac{d z_{5 t} d \check{z}_{5} d z_{6 t} d \check{z}_{6}}{\left(z_{56 t}^{2}+\check{z}_{56}^{2}\right)^{2}} \\
& \times\left(\frac{z_{56 t}^{2}+\check{z}_{56}^{2}}{\left[\left(x_{3}-z_{5}\right)_{t}^{2}+\check{z}_{5}^{2}\right]\left[\left(x_{4}-z_{6}\right)_{t}^{2}+\check{z}_{6}^{2}\right]}\right)^{\frac{1}{2}-i \nu_{3}} \mathcal{W}^{a}\left(z_{5 t} e_{t}+\check{z}_{5}, z_{6 t} e_{t}+\check{z}_{6}\right)
\end{aligned}
$$

where $\mathcal{W}$ is the conformal dipole with Wilson lines parallel to $n_{3}$ and

$$
\check{e} \equiv\left(n_{1} \sqrt{\frac{s_{23}}{s_{12} s_{13}}}-n_{2} \sqrt{\frac{s_{13}}{s_{12} s_{23}}}\right), \quad \check{s} \equiv \frac{s_{12} s_{23}}{s_{13}}
$$

After three evolutions (6.10), (6.12), and (6.15) we get the correlator of three dipoles

$$
\left\langle\mathcal{U}^{a}\left(z_{1 t} e_{t}+\tilde{z}_{1} \tilde{e}, z_{2 t} e_{t}+\tilde{z}_{2} \tilde{e}\right) \mathcal{V}^{a}\left(z_{3 t} e_{t}+\breve{z}_{3} \breve{e}, z_{4 t} e_{t}+\breve{z}_{4} \breve{e}\right) \hat{\mathcal{W}}^{a}\left(z_{5 t} e_{t}+\check{z}_{5} \check{e}_{2}, z_{6 t} e_{t}+\check{z}_{6} \check{e}\right)\right\rangle
$$

with Wilson lines parallel to $n_{1}, n_{2}, n_{3}$ and rapidity cutoffs $Y_{1}^{\prime}, Y_{2}^{\prime}, Y_{3}^{\prime}$. Moreover, one can think about color dipoles $\mathcal{U}^{Y_{1}^{\prime}}, \mathcal{V}^{Y_{2}^{\prime}}$, and $\mathcal{W}^{Y_{3}^{\prime}}$ as long Wilson frames with lengths $l_{1}^{\prime}=e^{Y_{i}^{\prime}} \sqrt{m_{\perp}^{2} \tilde{s}}$ etc., see figure $7 \mathrm{~b}$. We start the evolution with very long frames and evolve with lengths of these frames. We should stop the evolution if an extra loop in diagrams in figure $8 \mathrm{~b}$ and figure $8 \mathrm{c}$ does not bring an additional BFKL logarithm in comparison to the tree diagram in figure 8a. This happens when the relative energy of each pair of 

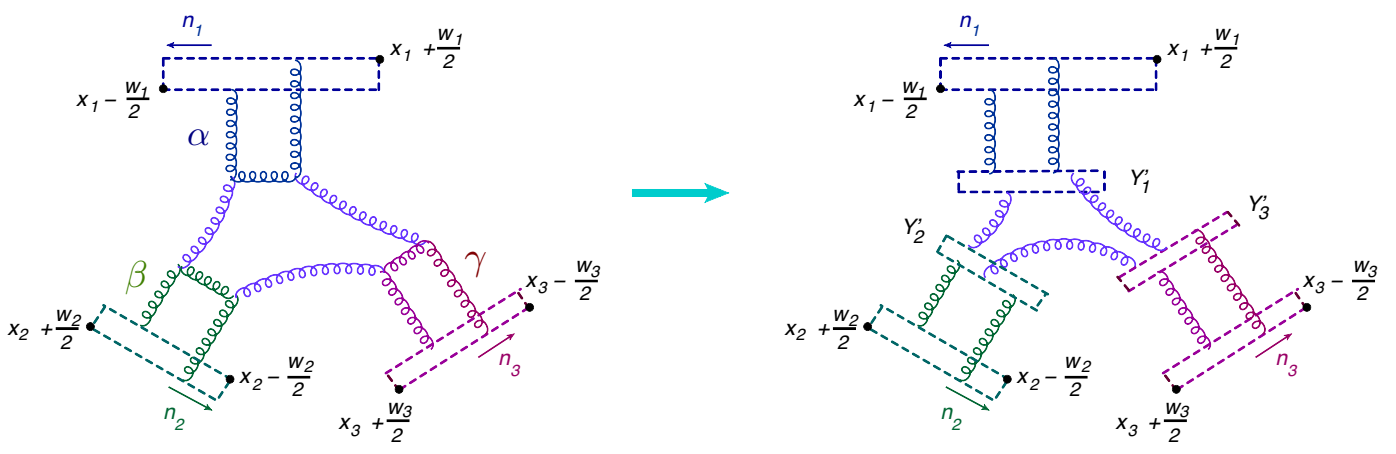

Figure 9. Three BFKL loops.

dipoles becomes compatible with $m_{\perp}$, or, in the coordinate space language, when the characteristic longitudinal distances are of the same order as the transverse ones so the BFKL approximations break down. For typical diagrams like in figure $8 \mathrm{~b}$ or $\mathrm{c}$ the characteristic longitudinal separations are $\sim l_{i}^{\prime} l_{j}^{\prime} s_{i j}$ so the condition is $\sim l_{i}^{\prime} l_{j}^{\prime} \geq \frac{1}{m_{\perp}^{2} s_{i j}}$. Thus, the three BFKL evolutions in diagrams in figure $7 \mathrm{~b}$ terminate at the rapidities

$$
\begin{aligned}
& l_{1}^{\prime} l_{2}^{\prime} \geq \frac{1}{m_{\perp}^{2} s_{12}} \quad \Leftrightarrow \quad \ln l_{1}^{\prime}+\ln l_{2}^{\prime} \geq-\ln m_{\perp}^{2} s_{12} \quad \Leftrightarrow \quad Y_{1}^{\prime}+Y_{2}^{\prime} \geq 0 \\
& l_{1}^{\prime} l_{3}^{\prime} \geq \frac{1}{m_{\perp}^{2} s_{13}} \Leftrightarrow \ln l_{1}^{\prime}+\ln l_{3}^{\prime} \geq-\ln m_{\perp}^{2} s_{13} \quad \Leftrightarrow \quad Y_{1}^{\prime}+Y_{3}^{\prime} \geq 0 \\
& l_{2}^{\prime} l_{3}^{\prime} \geq \frac{1}{m_{\perp}^{2} s_{23}} \quad \Leftrightarrow \quad \ln l_{2}^{\prime}+\ln l_{3}^{\prime} \geq-\ln m_{\perp}^{2} s_{23} \quad \Leftrightarrow \quad Y_{2}^{\prime}+Y_{3}^{\prime} \geq 0
\end{aligned}
$$

where $Y_{1}^{\prime}=\ln l_{1}^{\prime}+\frac{1}{2} \ln \tilde{s} m_{\perp}^{2}, Y_{2}^{\prime}=\ln l_{2}^{\prime}+\frac{1}{2} \ln \breve{s} m_{\perp}^{2}$, and $Y_{3}^{\prime}=\ln l_{3}^{\prime}+\frac{1}{2} \ln \check{s} m_{\perp}^{2}$. We see that the rapidity at which we stop the evolution of the $n_{1}$ dipole depends on the where we have terminated the evolutions of the second and third dipole which means that we need to integrate over all possible choices of "rapidity stops" $Y_{i}^{\prime}$ :

$$
\begin{aligned}
& \left\langle\mathcal{U}_{\text {conf }}^{Y_{1}}\left(x_{1 t},-\nu_{1}\right) \mathcal{V}_{\text {conf }}^{Y_{2}}\left(x_{2 t},-\nu_{2}\right) \mathcal{W}_{\text {conf }}^{Y_{3}}\left(x_{3 t},-\nu_{3}\right)\right\rangle^{\text {Fig. } 9} \\
& =\frac{1}{4} \aleph\left(\nu_{1}\right) \aleph\left(\nu_{2}\right) \aleph\left(\nu_{3}\right) \int_{-\infty}^{Y_{1}} d Y_{1}^{\prime} \int_{-\infty}^{Y_{2}} d Y_{2}^{\prime} \int_{-\infty}^{Y_{3}} d Y_{3}^{\prime} \theta\left(Y_{1}^{\prime}+Y_{2}^{\prime}\right) \theta\left(Y_{1}^{\prime}+Y_{3}^{\prime}\right) \theta\left(Y_{2}^{\prime}+Y_{3}^{\prime}\right) \\
& \quad \times e^{\left(Y_{1}-Y_{1}^{\prime}\right) \aleph\left(\nu_{3}\right)} e^{\left(Y_{2}-Y_{2}^{\prime}\right) \aleph\left(\nu_{3}\right)} e^{\left(Y_{3}-Y_{3}^{\prime}\right) \aleph\left(\nu_{3}\right)}\left\langle\mathcal{U}_{\text {conf }}\left(x_{1 t},-\nu_{1}\right) \mathcal{V}_{\text {conf }}\left(x_{2 t},-\nu_{2}\right) \mathcal{W}_{\text {conf }}\left(x_{3 t},-\nu_{3}\right)\right\rangle^{\text {tree }}
\end{aligned}
$$

The weight of the integrations can be figured out from the evolution equations for conformal dipoles up to an overall constant which will be determined later to be $\frac{1}{4}$. The factors $\aleph\left(\nu_{1}\right) \aleph\left(\nu_{2}\right) \aleph\left(\nu_{3}\right)$ can be understood by considering the lowest-order diagram with three BFKL evolutions shown in figure 9. The three integrations over $\alpha, \beta$, and $\gamma$ in figure 9 for conformal dipoles bring $\aleph\left(\nu_{i}\right) \int d Y_{i}$ for each of them so we get

$$
\begin{aligned}
& \left\langle\mathcal{U}_{\text {conf }}^{Y_{1}}\left(x_{1 t},-\nu_{1}\right) \mathcal{V}_{\text {conf }}^{Y_{2}}\left(x_{2 t},-\nu_{2}\right) \mathcal{W}_{\text {conf }}^{Y_{3}}\left(x_{3 t},-\nu_{3}\right)\right\rangle \\
& \sim \aleph\left(\nu_{1}\right) \aleph\left(\nu_{2}\right) \aleph\left(\nu_{3}\right) \int_{-\infty}^{Y_{1}} d Y_{1}^{\prime} \int_{-\infty}^{Y_{2}} d Y_{2}^{\prime} \int_{-\infty}^{Y_{3}} d Y_{3}^{\prime} \theta\left(Y_{1}^{\prime}+Y_{2}^{\prime}\right) \theta\left(Y_{1}^{\prime}+Y_{3}^{\prime}\right) \theta\left(Y_{2}^{\prime}+Y_{3}^{\prime}\right) \\
& \quad \times\left\langle\mathcal{U}_{\text {conf }}\left(x_{1 t},-\nu_{1}\right) \mathcal{V}_{\text {conf }}\left(x_{2 t},-\nu_{2}\right) \mathcal{W}_{\text {conf }}\left(x_{3 t},-\nu_{3}\right)\right\rangle^{\text {tree }}
\end{aligned}
$$


Combining the equations (6.10), (6.12), (6.15) and eq. (6.20) we get

$$
\begin{aligned}
\left\langle\mathcal{F}_{n_{1}}^{j_{1}}\left(x_{1 t}+\frac{w_{1 t}}{2}, x_{1 t}-\frac{w_{1 t}}{2}\right) \mathcal{F}_{n_{2}}^{j_{2}}\left(x_{2 t}+\frac{w_{2 t}}{2}, x_{2 t}-\frac{w_{2 t}}{2}\right) \mathcal{F}_{n_{3}}^{j_{3}}\left(x_{3 t}+\frac{w_{3 t}}{2}, x_{3 t}-\frac{w_{3 t}}{2}\right)\right\rangle \\
=-i \frac{N_{c}^{6}}{\pi^{9}} \int_{0}^{\infty} \prod_{n=1}^{3} \frac{d l_{n}}{l_{n}} l_{n}^{-\omega_{n}} \int \prod_{k=1}^{3} d \nu_{k} \frac{\nu_{k} 2^{-4 i \nu_{k}} \Gamma\left(\frac{3}{2}+i \nu_{k}\right) \Gamma\left(1-i \nu_{k}\right)}{\Gamma\left(\frac{3}{2}-i \nu_{k}\right) \Gamma\left(1+i \nu_{k}\right)}\left(w_{k t}^{2}\right)^{-\frac{1}{2}+i \nu_{k}} \\
\quad \times \frac{1}{4} \aleph\left(\nu_{1}\right) \aleph\left(\nu_{2}\right) \aleph\left(\nu_{3}\right) \int_{-\infty}^{Y_{1}} d Y_{1}^{\prime} \int_{-\infty}^{Y_{2}} d Y_{2}^{\prime} \int_{-\infty}^{Y_{3}} d Y_{3}^{\prime} \theta\left(Y_{1}^{\prime}+Y_{2}^{\prime}\right) \theta\left(Y_{1}^{\prime}+Y_{3}^{\prime}\right) \theta\left(Y_{2}^{\prime}+Y_{3}^{\prime}\right) \\
\quad \times e^{\left(Y_{1}-Y_{1}^{\prime}\right) \aleph\left(\nu_{1}\right)} e^{\left(Y_{2}-Y_{2}^{\prime}\right) \aleph\left(\nu_{3}\right)} e^{\left(Y_{3}-Y_{3}^{\prime}\right) \aleph\left(\nu_{3}\right)}\left\langle\mathcal{U}_{\text {conf }}\left(x_{1 t},-\nu_{1}\right) \mathcal{V}_{\text {conf }}\left(x_{2 t},-\nu_{2}\right) \mathcal{W}_{\text {conf }}\left(x_{3 t},-\nu_{3}\right)\right\rangle^{\text {tree }}
\end{aligned}
$$

This result is the integral over $\nu_{1}, \nu_{2}$, and $\nu_{3}$ of the product of longitudinal and transverse integrals which we will consider in turn.

\subsection{Longitudinal integrals}

Let us integrate the correlator (6.22) over $l_{i}$ according to the definition (4.1) of the "frame with spin j". Since

$$
\int_{0}^{\infty} l_{1}^{-\omega_{1}-1}=\int_{-\infty}^{\infty} d Y_{1} e^{-\omega_{1} Y_{1}}\left(\tilde{s} m_{\perp}^{2}\right)^{\frac{\omega_{1}}{2}}
$$

(and similarly for $Y_{2}$ and $Y_{3}$ ) we get

$$
\begin{aligned}
& \int_{-\infty}^{\infty} d Y_{1} d Y_{2} d Y_{3} e^{-\omega_{1} Y_{1}-\omega_{2} Y_{2}-\omega_{3} Y_{3}}\left(\tilde{s} m_{\perp}^{2}\right)^{\frac{\omega_{1}}{2}}\left(\breve{s} m_{\perp}^{2}\right)^{\frac{\omega_{2}}{2}}\left(\check{s} m_{\perp}^{2}\right)^{\frac{\omega_{3}}{2}} \\
& \quad \times \int_{-\infty}^{Y_{1}} d Y_{1}^{\prime} \int_{-\infty}^{Y_{2}} d Y_{2}^{\prime} \int_{-\infty}^{Y_{3}} d Y_{3}^{\prime} \theta\left(Y_{1}^{\prime}+Y_{2}^{\prime}\right) \theta\left(Y_{1}^{\prime}+Y_{3}^{\prime}\right) \theta\left(Y_{2}^{\prime}+Y_{3}^{\prime}\right) \\
& \quad \times e^{\left(Y_{1}-Y_{1}^{\prime}\right) \aleph\left(\nu_{1}\right)} e^{\left(Y_{2}-Y_{2}^{\prime}\right) \aleph\left(\nu_{3}\right)} e^{\left(Y_{3}-Y_{3}^{\prime}\right) \aleph\left(\nu_{3}\right)} \\
& =\frac{4 s_{12}^{\frac{\omega_{1}+\omega_{2}-\omega_{3}}{2}} s_{13}^{\frac{\omega_{1}+\omega_{3}-\omega_{2}}{2}} s_{23}^{\frac{\omega_{2}+\omega_{3}-\omega_{1}}{2}} m_{\perp}^{-\omega_{1}-\omega_{2}-\omega_{3}}}{\left(\omega_{1}+\omega_{2}-\omega_{3}\right)\left(\omega_{1}+\omega_{3}-\omega_{2}\right)\left(\omega_{2}+\omega_{3}-\omega_{1}\right)\left[\omega_{1}-\aleph\left(\nu_{1}\right)\right]\left[\omega_{2}-\aleph\left(\nu_{2}\right)\right]\left[\omega_{3}-\aleph\left(\nu_{3}\right)\right]} \\
& \rightarrow \frac{4\left(\frac{s_{12}}{x_{12 t}^{2}}\right)^{\frac{\omega_{1}+\omega_{2}-\omega_{3}}{2}}\left(\frac{s_{13}}{x_{13 t}^{2}}\right)^{\frac{\omega_{1}+\omega_{3}-\omega_{2}}{2}}\left(\frac{s_{23}}{x_{23 t}^{2}}\right)^{\frac{\omega_{2}+\omega_{3}-\omega_{1}}{2}}}{\left(\omega_{1}+\omega_{2}-\omega_{3}\right)\left(\omega_{1}+\omega_{3}-\omega_{2}\right)\left(\omega_{2}+\omega_{3}-\omega_{1}\right)\left[\omega_{1}-\aleph\left(\nu_{1}\right)\right]\left[\omega_{2}-\aleph\left(\nu_{2}\right)\right]\left[\omega_{3}-\aleph\left(\nu_{3}\right)\right]}
\end{aligned}
$$

We have replaced the transverse scale $m_{\perp}^{-2}$ by $x_{i j t}^{2}$ in accordance with general formula (3.9) and the result (B.10) of explicit first-order calculation performed in appendix B. ${ }^{13}$

Also, as discussed in ref. [27], the singularities $\left(\omega_{i}-\omega_{j}-\omega_{k}\right)^{-1}$ are of general nature since they arise from the fact that the correlator of three Wilson frames (4.3) acquires boost invariance as $n_{j} \rightarrow n_{k}$. This property is discussed in appendix C.

\footnotetext{
${ }^{13}$ This replacement is within the LLA accuracy and, moreover, I think that at the NLO level one will get the third line in eq. (6.23) similarly to the case of the two-frame correlator considered in ref. [17] where the calculation at the NLO BFKL level reproduces the correct arguments required by general formula (3.8).
} 


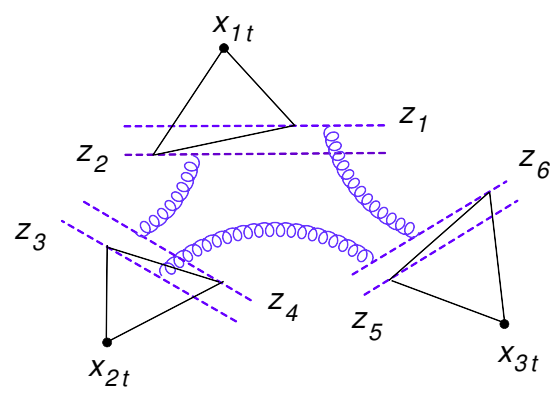

Figure 10. The structure of correlator (6.25). Straight lines denote "propagators" $\left(z_{i j}^{2}\right)^{\lambda}$ or $\left(\left(x_{i}-z_{j}\right)^{2}\right)^{\lambda}$.

\subsection{Transverse integral}

Using eq. (6.23) of previous section, one can rewrite the result (6.22) as

$$
\begin{aligned}
& \left\langle\mathcal{F}_{n_{1}}^{j_{1}}\left(x_{1 t}+\frac{w_{1 t}}{2}, x_{1 t}-\frac{w_{1 t}}{2}\right) \mathcal{F}_{n_{2}}^{j_{2}}\left(x_{2 t}+\frac{w_{2 t}}{2}, x_{2 t}-\frac{w_{2 t}}{2}\right) \mathcal{F}_{n_{3}}^{j_{3}}\left(x_{3 t}+\frac{w_{3 t}}{2}, x_{3 t}-\frac{w_{3 t}}{2}\right)\right\rangle \\
& =-i \frac{N_{c}^{6}}{\pi^{9}} \int d \nu_{1} d \nu_{2} d \nu_{3} \prod_{k=1}^{3} \frac{\nu_{k} 2^{-4 i \nu_{k}} \Gamma\left(\frac{3}{2}+i \nu_{k}\right) \Gamma\left(1-i \nu_{k}\right)}{\Gamma\left(\frac{3}{2}-i \nu_{k}\right) \Gamma\left(1+i \nu_{k}\right)}\left(w_{k t}^{2}\right)^{-\frac{1}{2}+i \nu_{k}} \\
& \quad \times \frac{\aleph\left(\nu_{1}\right) \aleph\left(\nu_{2}\right) \aleph\left(\nu_{3}\right)\left(\frac{s_{12}}{x_{12}^{2}}\right)^{\frac{\omega_{1}+\omega_{2}-\omega_{3}}{2}}\left(\frac{s_{13}}{x_{13 t}^{2}}\right)^{\frac{\omega_{1}+\omega_{3}-\omega_{2}}{2}}\left(\frac{s_{23}}{x_{23 t}^{2}}\right)^{\frac{\omega_{2}+\omega_{3}-\omega_{1}}{2}}}{\left(\omega_{1}+\omega_{2}-\omega_{3}\right)\left(\omega_{1}+\omega_{3}-\omega_{2}\right)\left(\omega_{2}+\omega_{3}-\omega_{1}\right)\left[\omega_{1}-\aleph\left(\nu_{1}\right)\right]\left[\omega_{2}-\aleph\left(\nu_{2}\right)\right]\left[\omega_{3}-\aleph\left(\nu_{3}\right)\right]} \\
& \quad \times\left\langle\mathcal{U}_{\text {conf }}^{Y_{1}^{\prime}}\left(x_{1 t},-\nu_{1}\right) \mathcal{V}_{\text {conf }}^{Y_{2}^{\prime}}\left(x_{2 t},-\nu_{2}\right) \mathcal{W}_{\text {conf }}^{Y_{3}^{\prime}}\left(x_{3 t},-\nu_{3}\right)\right\rangle^{\text {tree }}
\end{aligned}
$$

where the correlator of three conformal dipoles in the last line should be taken in the tree approximation.

To calculate this correlator, we rewrite conformal dipoles in terms of usual ones

$$
\begin{aligned}
\pi^{6} & \left\langle\mathcal{U}_{\text {conf }}^{Y_{1}^{\prime}}\left(x_{1 t},-\nu_{1}\right) \mathcal{V}_{\text {conf }}^{Y_{2}^{\prime}}\left(x_{2 t},-\nu_{2}\right) \mathcal{W}_{\text {conf }}^{Y_{3}^{\prime}}\left(x_{3 t},-\nu_{3}\right)\right\rangle^{\text {tree }}=\int \frac{d z_{1 t} d \tilde{z}_{1} d z_{2 t} d \tilde{z}_{2}}{\left(z_{12 t}^{2}+\tilde{z}_{12}^{2}\right)^{2}} \\
& \times \int \frac{d z_{3 t} d \breve{z}_{3} d z_{4 t} d \breve{z}_{4}}{\left(z_{34 t}^{2}+\breve{z}_{34}^{2}\right)^{2}} \frac{d z_{5 t} d \check{z}_{5} d z_{6 t} d \check{z}_{6}}{\left(z_{56 t}^{2}+\check{z}_{56}^{2}\right)^{2}}\left(\frac{z_{12 t}^{2}+\tilde{z}_{12}^{2}}{\left[\left(x_{1}-z_{1}\right)_{t}^{2}+\tilde{z}_{1}^{2}\right]\left[\left(x_{1}-z_{2}\right)_{t}^{2}+\tilde{z}_{2}^{2}\right]}\right)^{\frac{1}{2}+i \nu_{1}} \\
& \times\left(\frac{z_{34 t}^{2}+\breve{z}_{34}^{2}}{\left[\left(x_{2}-z_{3}\right)_{t}^{2}+\breve{z}_{3}^{2}\right]\left[\left(x_{2}-z_{4}\right)_{t}^{2}+\breve{z}_{4}^{2}\right]}\right)^{\frac{1}{2}+i \nu_{2}}\left(\frac{z_{56 t}^{2}+\check{z}_{56}^{2}}{\left[\left(x_{3}-z_{5}\right)_{t}^{2}+\check{z}_{5}^{2}\right]\left[\left(x_{3}-z_{6}\right)_{t}^{2}+\check{z}_{6}^{2}\right]}\right)^{\frac{1}{2}+i \nu_{3}} \\
& \times\left\langle\mathcal{U}\left(z_{1 t} e_{t}+\tilde{z}_{1} \tilde{e}, z_{2 t} e_{t}+\tilde{z}_{2} \tilde{e}\right) \mathcal{V}\left(z_{3 t} e_{t}+\breve{z}_{3} \breve{e}, z_{4 t} e_{t}+\breve{z}_{4} \breve{e}\right) \hat{\mathcal{W}}\left(z_{5 t} e_{t}+\check{z}_{5} \check{e}, z_{6 t} e_{t}+\check{z}_{6} \check{e}\right)\right\rangle
\end{aligned}
$$

This integral is illustrated on figure 10 .

Using the leading-order correlator

$$
\left\langle\left[\infty n_{1}+x,-\infty n_{1}+x\right]\left[\infty n_{2}+y,-\infty n_{2}+y\right]\right\rangle=i \alpha_{s} t^{a} \otimes t^{a} \ln \left[(x-y)^{2}-2 \frac{(x-y)_{n_{1}}(x-y)_{n_{2}}}{n_{1} \cdot n_{2}}\right]
$$


one easily obtains

$$
\begin{aligned}
\left\langle U\left(z_{1 t} e_{t}+\tilde{z}_{1} \tilde{e}\right) V\left(z_{3 t} e_{t}+\breve{z}_{3} \breve{e}\right)\right\rangle & =i \alpha_{s} t^{a} \otimes t^{a} \ln \left[z_{13 t}^{2}+\left(\tilde{z}_{1}+\breve{z}_{3}\right)^{2}\right] \\
\left\langle U\left(z_{1 t} e_{t}+\tilde{z}_{1} \tilde{e}\right) W\left(z_{5 t} e_{t}+\check{z}_{5} \check{e}\right)\right\rangle & =i \alpha_{s} t^{a} \otimes t^{a} \ln \left[z_{15 t}^{2}+\left(\tilde{z}_{1}+\check{z}_{5}\right)^{2}\right] \\
\left\langle V\left(z_{3 t} e_{t}+\breve{z}_{3} \breve{e}\right) W\left(z_{5 t} e_{t}+\check{z}_{5} \check{e}\right)\right\rangle & =i \alpha_{s} t^{a} \otimes t^{a} \ln \left[z_{35 t}^{2}+\left(\breve{z}_{3}+\check{z}_{5}\right)^{2}\right]
\end{aligned}
$$

and therefore the tree-level correlator of three color dipoles reads

$$
\begin{aligned}
& \left\langle\mathcal{U}^{a}\left(z_{1 t} e_{t}+\tilde{z}_{1} \tilde{e}, z_{2 t} e_{t}+\tilde{z}_{2} \tilde{e}\right) \mathcal{V}^{a}\left(z_{3 t} e_{t}+\breve{z}_{3} \breve{e}_{,} z_{4 t} e_{t}+\breve{z}_{4} \breve{e}\right) \hat{\mathcal{W}}^{a}\left(z_{5 t} e_{t}+\check{z}_{5} \check{e}_{,}, z_{6 t} e_{t}+\check{z}_{6} \check{e}\right)\right\rangle \\
& =-i \alpha_{s}^{3} \frac{N_{c}^{2}-1}{N_{c}^{3}} \ln \frac{\left[z_{13 t}^{2}+\left(\tilde{z}_{1}+\breve{z}_{3}\right)^{2}\right]\left[z_{24 t}^{2}+\left(\tilde{z}_{2}+\breve{z}_{4}\right)^{2}\right]}{\left[z_{14 t}^{2}+\left(\tilde{z}_{1}+\breve{z}_{4}\right)^{2}\right]\left[z_{23 t}^{2}+\left(\tilde{z}_{2}+\breve{z}_{3}\right)^{2}\right]} \\
& \quad \times \ln \frac{\left[z_{15 t}^{2}+\left(\tilde{z}_{1}+\check{z}_{5}\right)^{2}\right]\left[z_{26 t}^{2}+\left(\tilde{z}_{2}+\check{z}_{6}\right)^{2}\right]}{\left[z_{16 t}^{2}+\left(\tilde{z}_{1}+\check{z}_{6}\right)^{2}\right]\left[z_{25 t}^{2}+\left(\tilde{z}_{2}+\check{z}_{5}\right)^{2}\right]} \ln \frac{\left[z_{35 t}^{2}+\left(\breve{z}_{3}+\check{z}_{5}\right)^{2}\right]\left[z_{46 t}^{2}+\left(\breve{z}_{4}+\check{z}_{6}\right)^{2}\right]}{\left[z_{36 t}^{2}+\left(\breve{z}_{3}+\check{z}_{6}\right)^{2}\right]\left[z_{45 t}^{2}+\left(\breve{z}_{4}+\check{z}_{5}\right)^{2}\right]}
\end{aligned}
$$

One obtains

$$
\begin{aligned}
& \left\langle\mathcal{U}_{\text {conf }}\left(x_{1 t},-\nu_{1}\right) \mathcal{V}_{\text {conf }}\left(x_{2 t},-\nu_{2}\right) \mathcal{W}_{\text {conf }}\left(x_{3 t},-\nu_{3}\right)\right\rangle^{\text {tree }} \\
& \quad=-i \alpha_{s}^{3} \frac{N_{c}^{2}-1}{N_{c}^{3} \pi^{6}} I\left(x_{1 t}, x_{2 t}, x_{3 t} ; \nu_{1}, \nu_{2}, \nu_{3}\right)
\end{aligned}
$$

where

$$
\begin{aligned}
I & \left(x_{1 t}, x_{2 t}, x_{3 t} ; \nu_{1}, \nu_{2}, \nu_{3}\right)=\int \frac{d z_{1 t} d \tilde{z}_{1} d z_{2 t} d \tilde{z}_{2}}{\left(z_{12 t}^{2}+\tilde{z}_{12}^{2}\right)^{2}} \frac{d z_{3 t} d \breve{z}_{3} d z_{4 t} d \breve{z}_{4}}{\left(z_{34 t}^{2}+\breve{z}_{34}^{2}\right)^{2}} \frac{d z_{5 t} d \check{z}_{5} d z_{6 t} d \check{z}_{6}}{\left(z_{56 t}^{2}+\check{z}_{56}^{2}\right)^{2}} \\
\quad & \times\left(\frac{z_{12 t}^{2}+\tilde{z}_{12}^{2}}{\left[\left(x_{1}-z_{1}\right)_{t}^{2}+\tilde{z}_{1}^{2}\right]\left[\left(x_{1}-z_{2}\right)_{t}^{2}+\tilde{z}_{2}^{2}\right]}\right)^{\frac{1}{2}+i \nu_{1}}\left(\frac{z_{34 t}^{2}+\breve{z}_{34}^{2}}{\left[\left(x_{2}-z_{3}\right)_{t}^{2}+\breve{z}_{3}^{2}\right]\left[\left(x_{2}-z_{4}\right)_{t}^{2}+\breve{z}_{4}^{2}\right]}\right)^{\frac{1}{2}+i \nu_{2}} \\
\quad & \times\left(\frac{z_{56 t}^{2}+\check{z}_{56}^{2}}{\left[\left(x_{3}-z_{5}\right)_{t}^{2}+\check{z}_{5}^{2}\right]\left[\left(x_{3}-z_{6}\right)_{t}^{2}+\check{z}_{6}^{2}\right]}\right)^{\frac{1}{2}+i \nu_{3}} \ln \frac{\left[z_{13 t}^{2}+\left(\tilde{z}_{1}+\breve{z}_{3}\right)^{2}\right]\left[z_{24 t}^{2}+\left(\tilde{z}_{2}+\breve{z}_{4}\right)^{2}\right]}{\left[z_{14 t}^{2}+\left(\tilde{z}_{1}+\breve{z}_{4}\right)^{2}\right]\left[z_{23 t}^{2}+\left(\tilde{z}_{2}+\breve{z}_{3}\right)^{2}\right]} \\
\quad & \times \ln \frac{\left[z_{15 t}^{2}+\left(\tilde{z}_{1}+\check{z}_{5}\right)^{2}\right]\left[z_{26 t}^{2}+\left(\tilde{z}_{2}+\check{z}_{6}\right)^{2}\right]}{\left[z_{16 t}^{2}+\left(\tilde{z}_{1}+\check{z}_{6}\right)^{2}\right]\left[z_{25 t}^{2}+\left(\tilde{z}_{2}+\check{z}_{5}\right)^{2}\right]} \ln \frac{\left[z_{35 t}^{2}+\left(\breve{z}_{3}+\check{z}_{5}\right)^{2}\right]\left[z_{46 t}^{2}+\left(\breve{z}_{4}+\check{z}_{6}\right)^{2}\right]}{\left[z_{36 t}^{2}+\left(\breve{z}_{3}+\check{z}_{6}\right)^{2}\right]\left[z_{45 t}^{2}+\left(\breve{z}_{4}+\check{z}_{5}\right)^{2}\right]}
\end{aligned}
$$

The structure of the integrations in this equation is the following: each conformal dipole evolves in its own "transverse plane" and the obtained dipoles interact by logarithmical correlators (6.27). Fortunately, the integral (6.30) coincides with an usual two-dimensional integral in the (formal) $(x, y)$ plane

$$
\begin{aligned}
& I\left(x_{1}, x_{2}, x_{3} ; \nu_{1}, \nu_{2}, \nu_{3}\right)=\int \frac{d^{2} z_{1} d^{2} z_{2}}{z_{12}^{4}} \frac{d^{2} z_{3} d^{2} z_{4}}{z_{34}^{4}} \frac{d^{2} z_{5} d^{2} z_{6}}{z_{56}^{4}} \\
& \quad \times\left(\frac{z_{12}^{2}}{\left(x_{1}-z_{1}\right)^{2}\left(x_{1}-z_{2}\right)^{2}}\right)^{\frac{1}{2}+i \nu_{1}}\left(\frac{z_{34}^{2}}{\left(x_{2}-z_{3}\right)^{2}\left(x_{2}-z_{4}\right)^{2}}\right)^{\frac{1}{2}+i \nu_{2}}\left(\frac{z_{56}^{2}}{\left(x_{3}-z_{5}\right)^{2}\left(x_{3}-z_{6}\right)^{2}}\right)^{\frac{1}{2}+i \nu_{2}} \\
& \quad \times \ln \frac{\left[z_{13 x}^{2}+\left(z_{1 y}+z_{3 y}\right)^{2}\right]\left[z_{24 x}^{2}+\left(z_{2 y}+z_{4 y}\right)^{2}\right]}{\left[z_{14 x}^{2}+\left(z_{1 y}+z_{4 y}\right)^{2}\right]\left[z_{23 x}^{2}+\left(z_{2 y}+z_{3 y}\right)^{2}\right]} \ln \frac{\left[z_{15 x}^{2}+\left(z_{1 y}+z_{5 y}\right)^{2}\right]\left[z_{2 x}^{2}+\left(z_{2 y}+z_{6 y}\right)^{2}\right]}{\left[z_{16 x}^{2}+\left(z_{1 y}+z_{6 y}\right)^{2}\right]\left[z_{25 x}^{2}+\left(z_{2 y}+z_{5 y}\right)^{2}\right]} \\
& \quad \times \ln \frac{\left[z_{35 x}^{2}+\left(z_{3 y}+z_{5 y}\right)^{2}\right]\left[z_{46 x}^{2}+\left(z_{4 y}+z_{6 y}\right)^{2}\right]}{\left[z_{36 x}^{2}+\left(z_{3 y}+z_{6 y}\right)^{2}\right]\left[z_{45 x}^{2}+\left(z_{4 y}+z_{5 y}\right)^{2}\right]}
\end{aligned}
$$

with $x_{1 y}=x_{2 y}=x_{3 y}=0$, see figure 11a. 


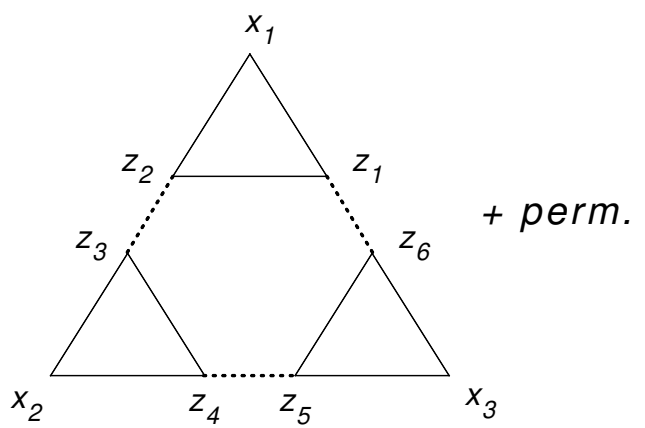

(a)

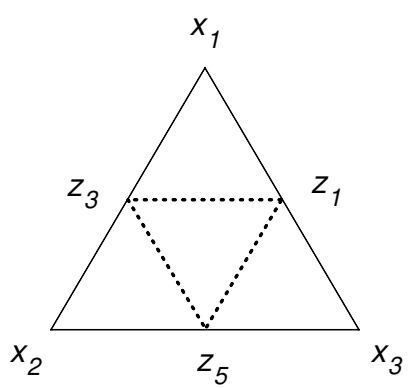

(b)

Figure 11. Transverse integral. Solid lines denote usual "propagators" of the type $\left(z_{i}-z_{j}\right)^{2 \lambda}$ and dotted lines expressions of the type $\left[\left(z_{i}-z_{j}\right)_{x}^{2}+\left(z_{i}+z_{j}\right)_{y}^{2}\right]^{\lambda}$.

Next, we use formula

$$
\int \frac{d^{2} z_{2}}{z_{12}^{4}}\left(\frac{z_{12}^{2}}{\left(x_{1}-z_{1}\right)^{2}\left(x_{1}-z_{2}\right)^{2}}\right)^{\frac{1}{2}+i \nu_{1}} \ln z_{23}^{2}=\frac{\pi\left[\left(x_{1}-z_{1}\right)^{2}\right]^{-\frac{3}{2}-i \nu_{1}}}{\left(\frac{1}{2}-i \nu_{1}\right)^{2}}\left[\left(\frac{\left(x_{1}-z_{3}\right)^{2}}{z_{13}^{2}}\right)^{\frac{1}{2}-i \nu_{1}}-1\right]
$$

and rewrite it by changing sign of $z_{3 y}$ as

$$
\begin{aligned}
& \int \frac{d^{2} z_{2}}{z_{12}^{4}}\left(\frac{z_{12}^{2}}{\left(x_{1}-z_{1}\right)^{2}\left(x_{1}-z_{2}\right)^{2}}\right)^{\frac{1}{2}+i \nu} \ln \left[z_{23 x}^{2}+\left(z_{2 y}+z_{3 y}\right)^{2}\right] \\
& =\frac{\pi\left[\left(x_{1}-z_{1}\right)^{2}\right]^{-\frac{3}{2}-i \nu_{1}}}{\left(\frac{1}{2}-i \nu_{1}\right)^{2}}\left[\left(\frac{\left(x_{1}-z_{3}\right)^{2}}{z_{13 x}^{2}+\left(z_{1 y}+z_{3 y}\right)^{2}}\right)^{\frac{1}{2}-i \nu_{1}}-1\right]
\end{aligned}
$$

Using this integral and similar integrals for integration over $z_{4}$ and $z_{6}$ one gets after some algebra (see figure 11b)

$$
\begin{aligned}
I & \left(x_{1}, x_{2}, x_{3} ; \nu_{1}, \nu_{2}, \nu_{3}\right) \\
= & -\frac{8 \pi^{3}}{\left(\frac{1}{2}-i \nu_{1}\right)^{2}\left(\frac{1}{2}-i \nu_{2}\right)^{2}\left(\frac{1}{2}-i \nu_{3}\right)^{2}} \int \frac{d^{2} z_{1}}{\left(x_{1}-z_{1}\right)^{4}} \frac{d^{2} z_{3}}{\left(x_{2}-z_{3}\right)^{4}} \frac{d^{2} z_{5}}{\left(x_{3}-z_{5}\right)^{4}} \\
& \times\left[\frac{z_{13 x}^{2}+\left(z_{1 y}+z_{3 y}\right)^{2}}{\left(x_{1}-z_{1}\right)^{2}\left(x_{1}-z_{3}\right)^{2}}\right]^{-\frac{1}{2}+i \nu_{1}}\left[\frac{z_{35 x}^{2}+\left(z_{3 y}+z_{5 y}\right)^{2}}{\left(x_{2}-z_{3}\right)^{2}\left(x_{2}-z_{5}\right)^{2}}\right]^{-\frac{1}{2}+i \nu_{2}}\left[\frac{z_{15 x}^{2}+\left(z_{1 y}+z_{5 y}\right)^{2}}{\left(x_{3}-z_{1}\right)^{2}\left(x_{3}-z_{5}\right)^{2}}\right]^{-\frac{1}{2}+i \nu_{3}}
\end{aligned}
$$

To calculate this integral, we can take $x_{1}=0$ and perform the inversion $x_{i} \rightarrow \frac{x_{i}}{x^{2}}$ to obtain

$$
\begin{aligned}
I^{\text {inv }} & \left(x_{1}, x_{2}, x_{3} ; \nu_{1}, \nu_{2}, \nu_{3}\right) \\
= & -\frac{8 \pi^{3}\left(x_{2}^{2}\right)^{1+2 i \nu_{2}}\left(x_{3}^{2}\right)^{1+2 i \nu_{3}}}{\left(\frac{1}{2}-i \nu_{1}\right)^{2}\left(\frac{1}{2}-i \nu_{2}\right)^{2}\left(\frac{1}{2}-i \nu_{3}\right)^{2}} \int d^{2} z_{1} \frac{d^{2} z_{3}}{\left(x_{2}-z_{3}\right)^{4}} \frac{d^{2} z_{5}}{\left(x_{3}-z_{5}\right)^{4}} \\
& \times\left[z_{13 x}^{2}+\left(z_{1 y}+z_{3 y}\right)^{2}\right]^{-\frac{1}{2}+i \nu_{1}}\left[\frac{z_{35 x}^{2}+\left(z_{3 y}+z_{5 y}\right)^{2}}{\left(x_{2}-z_{3}\right)^{2}\left(x_{2}-z_{5}\right)^{2}}\right]^{-\frac{1}{2}+i \nu_{2}}\left[\frac{z_{15 x}^{2}+\left(z_{1 y}+z_{5 y}\right)^{2}}{\left(x_{3}-z_{1}\right)^{2}\left(x_{3}-z_{5}\right)^{2}}\right]^{-\frac{1}{2}+i \nu_{3}} \\
= & -\frac{8 \pi^{6}\left(x_{2}^{2}\right)^{1+2 i \nu_{2}}\left(x_{3}^{2}\right)^{1+2 i \nu_{3}}\left(x_{23}^{2}\right)^{-\frac{1}{2}+i\left(\nu_{1}-\nu_{2}-\nu_{3}\right)}}{\left(\frac{1}{2}-i \nu_{1}\right)^{2}\left(\frac{1}{2}-i \nu_{2}\right)^{2}\left(\frac{1}{2}-i \nu_{3}\right)^{2}} \Lambda\left(\nu_{1}, \nu_{2}, \nu_{3}\right)
\end{aligned}
$$


where

$$
\begin{aligned}
\Lambda\left(\nu_{1}, \nu_{2}, \nu_{3}\right) \equiv & \left(x_{23}^{2}\right)^{\frac{1}{2}-i\left(\nu_{1}-\nu_{2}-\nu_{3}\right)} \frac{1}{\pi^{3}} \int d^{2} z_{1} \frac{d^{2} z_{3}}{\left(x_{23}-z_{3}\right)^{4}} \frac{d^{2} z_{5}}{z_{5}^{4}} \\
& \times\left[z_{13 x}^{2}+\left(z_{1 y}+z_{3 y}\right)^{2}\right]^{-\frac{1}{2}+i \nu_{1}}\left[\frac{z_{35}^{2}}{\left(x_{23}-z_{3}\right)^{2}\left(x_{23}-z_{5}\right)^{2}}\right]^{-\frac{1}{2}+i \nu_{2}}\left[\frac{z_{15}^{2}}{z_{1}^{2} z_{5}^{2}}\right]^{-\frac{1}{2}+i \nu_{3}} \\
= & \frac{1}{\pi^{3}} \int d^{2} z_{1} \frac{d^{2} z_{3}}{\left(1-z_{3}\right)^{4}} \frac{d^{2} z_{5}}{z_{5}^{4}} \\
& \times\left[z_{13 x}^{2}+\left(z_{1 y}+z_{3 y}\right)^{2}\right]^{-\frac{1}{2}+i \nu_{1}}\left[\frac{z_{35}^{2}}{\left(1-z_{3}\right)^{2}\left(1-z_{5}\right)^{2}}\right]^{-\frac{1}{2}+i \nu_{2}}\left[\frac{z_{15}^{2}}{z_{1}^{2} z_{5}^{2}}\right]^{-\frac{1}{2}+i \nu_{3}}
\end{aligned}
$$

where " 1 " in the denominators stands for the vector $(1,0)$. This integral resembles the integral for function $\Omega$ defining three-pomeron vertex [31], only with "modified propagator" $\left[z_{13 x}^{2}+\left(z_{1 y}+z_{3 y}\right)^{2}\right]^{-\frac{1}{2}+i \nu_{1}}$ instead of usual $\left[z_{13 x}^{2}+z_{13 y}^{2}\right]^{-\frac{1}{2}+i \nu_{1}}$ in ref. [31]. The function $\Lambda\left(\nu_{1}, \nu_{2}, \nu_{3}\right)$ can be represented as four-fold Mellin-Barnes integral, see eq. (6.40) below and eq. (D.13) in appendix D.

Performing inversion of eq. (6.34) we get

$$
\begin{aligned}
I\left(x_{1 t}, x_{2 t}, x_{3 t} ; \nu_{1}, \nu_{2}, \nu_{3}\right)= & -8 \pi^{6} \frac{\Lambda\left(\nu_{1}, \nu_{2}, \nu_{3}\right)}{\left(\frac{1}{2}-i \nu_{1}\right)^{2}\left(\frac{1}{2}-i \nu_{2}\right)^{2}\left(\frac{1}{2}-i \nu_{3}\right)^{2}} \\
& \times\left(x_{12 t}^{2}\right)^{-\frac{1}{2}-i\left(\nu_{1}+\nu_{2}-\nu_{3}\right)}\left(x_{13 t}^{2}\right)^{-\frac{1}{2}-i\left(\nu_{1}+\nu_{3}-\nu_{2}\right)}\left(x_{23 t}^{2}\right)^{-\frac{1}{2}-i\left(\nu_{2}+\nu_{3}-\nu_{1}\right)}
\end{aligned}
$$

so that

$$
\begin{aligned}
& \left\langle\mathcal{U}_{\text {conf }}\left(x_{1 t},-\nu_{1}\right) \mathcal{V}_{\text {conf }}\left(x_{2 t},-\nu_{2}\right) \mathcal{W}_{\text {conf }}\left(x_{3 t},-\nu_{3}\right)\right\rangle^{\text {tree }} \\
& =8 i \alpha_{s}^{3} \frac{N_{c}^{2}-1}{N_{c}^{3}} \Lambda\left(\nu_{1}, \nu_{2}, \nu_{3}\right) \frac{\left(x_{12 t}^{2}\right)^{-\frac{1}{2}-i\left(\nu_{1}+\nu_{2}-\nu_{3}\right)}\left(x_{13 t}^{2}\right)^{-\frac{1}{2}-i\left(\nu_{1}+\nu_{3}-\nu_{2}\right)}\left(x_{23 t}^{2}\right)^{-\frac{1}{2}-i\left(\nu_{2}+\nu_{3}-\nu_{1}\right)}}{\left(\frac{1}{2}-i \nu_{1}\right)^{2}\left(\frac{1}{2}-i \nu_{2}\right)^{2}\left(\frac{1}{2}-i \nu_{3}\right)^{2}}
\end{aligned}
$$

\subsection{The result}

Substituting the transverse integral (6.37) into eq. (6.24) we get

$$
\begin{aligned}
& \left\langle\mathcal{F}_{n_{1}}^{j_{1}}\left(x_{1 t}+\frac{w_{1 t}}{2}, x_{1 t}-\frac{w_{1 t}}{2}\right) \mathcal{F}_{n_{2}}^{j_{2}}\left(x_{2 t}+\frac{w_{2 t}}{2}, x_{2 t}-\frac{w_{2 t}}{2}\right) \mathcal{F}_{n_{3}}^{j_{3}}\left(x_{3 t}+\frac{w_{3 t}}{2}, x_{3 t}-\frac{w_{3 t}}{2}\right)\right\rangle \\
& =\frac{64 g^{6}}{\pi^{6}}\left(N_{c}^{2}-1\right) \int_{-\infty}^{\infty} d \nu_{1} d \nu_{2} d \nu_{3} \prod_{k=1}^{3} \frac{\nu_{k} 2^{-4 i \nu_{k}} \Gamma\left(\frac{3}{2}+i \nu_{k}\right) \Gamma\left(1-i \nu_{k}\right)}{\Gamma\left(\frac{3}{2}-i \nu_{k}\right) \Gamma\left(1+i \nu_{k}\right)}\left(w_{k t}^{2}\right)^{-\frac{1}{2}+i \nu_{k}} \\
& \quad \times \frac{8 \aleph\left(\nu_{1}\right) \aleph\left(\nu_{2}\right) \aleph\left(\nu_{3}\right)\left(\frac{s_{12}}{x_{12 t}^{2}}\right)^{\frac{\omega_{1}+\omega_{2}-\omega_{3}}{2}}\left(\frac{s_{13}}{x_{13 t}^{2}}\right)^{\frac{\omega_{1}+\omega_{3}-\omega_{2}}{2}}\left(\frac{s_{23}}{x_{23 t}^{2}}\right)^{\frac{\omega_{2}+\omega_{3}-\omega_{1}}{2}}}{\left(\omega_{1}+\omega_{2}-\omega_{3}\right)\left(\omega_{1}+\omega_{3}-\omega_{2}\right)\left(\omega_{2}+\omega_{3}-\omega_{1}\right)\left[\omega_{1}-\aleph\left(\nu_{1}\right)\right]\left[\omega_{2}-\aleph\left(\nu_{2}\right)\right]\left[\omega_{3}-\aleph\left(\nu_{3}\right)\right]} \\
& \quad \times \frac{\left(x_{12 t}^{2}\right)^{-\frac{1}{2}-i\left(\nu_{1}+\nu_{2}-\nu_{3}\right)}\left(x_{13 t}^{2}\right)^{-\frac{1}{2}-i\left(\nu_{1}+\nu_{3}-\nu_{2}\right)}\left(x_{23 t}^{2}\right)^{-\frac{1}{2}-i\left(\nu_{2}+\nu_{3}-\nu_{1}\right)}}{\left(\frac{1}{2}-i \nu_{1}\right)^{2}\left(\frac{1}{2}-i \nu_{2}\right)^{2}\left(\frac{1}{2}-i \nu_{3}\right)^{2}} \Lambda\left(\nu_{2}, \nu_{3}\right)
\end{aligned}
$$


To estimate this integral at small $\omega$ 's it is convenient to rewrite it in variables $\gamma_{i}=2 i \nu_{i}-1$. Defining $\tilde{\aleph}\left(\gamma_{i}\right) \equiv \aleph\left(\nu_{i}\right)$ and $\tilde{\Lambda}\left(\gamma_{i}\right) \equiv \Lambda\left(\nu_{i}\right)$ we get

$$
\begin{aligned}
\left\langle\mathcal{F}_{n_{1}}^{j_{1}}\left(x_{1 t}+\frac{w_{1 t}}{2}, x_{1 t}-\frac{w_{1 t}}{2}\right) \mathcal{F}_{n_{2}}^{j_{2}}\left(x_{2 t}+\frac{w_{2 t}}{2}, x_{2 t}-\frac{w_{2 t}}{2}\right) \mathcal{F}_{n_{3}}^{j_{3}}\left(x_{3 t}+\frac{w_{3 t}}{2}, x_{3 t}-\frac{w_{3 t}}{2}\right)\right\rangle \\
=-\frac{8 g^{6}\left(N_{c}^{2}-1\right)}{\pi^{6} x_{12 t}^{2} x_{13 t}^{2} x_{23 t}^{2}} \frac{\left(\frac{s_{12}}{x_{12 t}^{2}}\right)^{\frac{\omega_{1}+\omega_{2}-\omega_{3}}{2}}\left(\frac{s_{13}}{x_{13 t}^{2}}\right)^{\frac{\omega_{1}+\omega_{3}-\omega_{2}}{2}}\left(\frac{s_{23}}{x_{23 t}^{2}}\right)^{\frac{\omega_{2}+\omega_{3}-\omega_{1}}{2}}}{\left(\omega_{1}+\omega_{2}-\omega_{3}\right)\left(\omega_{1}+\omega_{3}-\omega_{2}\right)\left(\omega_{2}+\omega_{3}-\omega_{1}\right)} \\
\quad \times \int_{-1-i \infty}^{-1+i \infty} d \gamma_{1} d \gamma_{2} d \gamma_{3} \prod_{k=1}^{3} \frac{\left(1+\gamma_{k}\right) 2^{-2 \gamma_{k}} \Gamma\left(2+\frac{\gamma_{k}}{2}\right) \Gamma\left(\frac{1}{2}-\frac{\gamma_{k}}{2}\right)}{\Gamma\left(1-\frac{\gamma_{k}}{2}\right) \Gamma\left(\frac{3}{2}+\frac{\gamma_{k}}{2}\right) \gamma_{k}^{2}} \\
\quad \times \frac{\tilde{\aleph}\left(\gamma_{1}\right) \tilde{\aleph}\left(\gamma_{2}\right) \tilde{\aleph}\left(\gamma_{3}\right) \tilde{\Lambda}\left(\gamma_{1}, \gamma_{2}, \gamma_{3}\right)}{\left[\omega_{1}-\tilde{\aleph}\left(\gamma_{1}\right)\right]\left[\omega_{2}-\tilde{\aleph}\left(\gamma_{2}\right)\right]\left[\omega_{3}-\tilde{\aleph}\left(\gamma_{3}\right)\right]}\left(\frac{w_{1 t}^{2} x_{23 t}^{2}}{x_{12 t}^{2} x_{13 t}^{2}}\right)^{\frac{\gamma_{1}}{2}}\left(\frac{w_{2 t}^{2} x_{13 t}^{2}}{x_{12 t}^{2} x_{23 t}^{2}}\right)^{\frac{\gamma_{2}}{2}}\left(\frac{w_{3 t}^{2} x_{12 t}^{2}}{x_{13 t}^{2} x_{23 t}^{2}}\right)^{\frac{\gamma_{3}}{2}}
\end{aligned}
$$

where contours over real $\nu_{i}$ transform to the contours parallel to imaginary axis since $\gamma_{i}=2 i \nu_{i}-1$. The function $\tilde{\Lambda}\left(\gamma_{i}\right)$ (defined by eq. (6.35)) is represented in appendix D as

$$
\bar{\Lambda}\left(\epsilon_{1}, \epsilon_{2}, \epsilon_{3}\right)=-\frac{\sin ^{2} \pi \epsilon_{2} \sin ^{2} \pi \epsilon_{3}}{\pi^{3} \sin \pi\left(\epsilon_{2}+\epsilon_{3}\right) \Gamma^{2}\left(\epsilon_{1}\right)} I\left(\epsilon_{1}, \epsilon_{2}, \epsilon_{3}\right)
$$

where $\bar{\Lambda}\left(\epsilon_{i}\right) \equiv \tilde{\Lambda}\left(\gamma_{i}\right), \epsilon_{i} \equiv-\frac{\gamma_{i}}{2}$ and

$$
\begin{aligned}
& I\left(\epsilon_{1}, \epsilon_{2}, \epsilon_{3}\right) \\
& =\int_{C} \frac{d s_{1} d s_{2} d s_{3} d s_{4}}{(2 \pi i)^{4}} \Gamma\left(\epsilon_{1}-s_{1}-s_{2}\right) \Gamma\left(s_{1}\right) \Gamma\left(s_{2}\right) \Gamma\left(\epsilon_{1}-s_{3}-s_{4}\right) \Gamma\left(s_{3}\right) \Gamma\left(s_{4}\right) \\
& \quad \times \frac{\Gamma\left(\epsilon_{2}-1-s_{1}\right) \Gamma\left(s_{1}+1\right)}{\Gamma\left(\epsilon_{2}\right)} \frac{\Gamma\left(1+\epsilon_{3}-s_{3}\right) \Gamma\left(-1+s_{3}\right)}{\Gamma\left(\epsilon_{3}\right)} \\
& \quad \times \frac{\Gamma\left(\epsilon_{2}-1-s_{4}\right) \Gamma\left(1-\epsilon_{1}-\epsilon_{2}+s_{3}+s_{4}\right)}{\Gamma\left(-\epsilon_{1}+s_{3}\right)} \\
& \quad \times \frac{\Gamma\left(1+\epsilon_{3}-s_{2}\right) \Gamma\left(1-\epsilon_{1}-\epsilon_{3}+s_{1}+s_{2}\right)}{\Gamma\left(2-\epsilon_{1}+s_{1}\right)} \frac{\Gamma\left(\epsilon_{2}-s_{1}\right) \Gamma\left(\epsilon_{3}-s_{3}\right)}{\Gamma\left(\epsilon_{2}+\epsilon_{3}-s_{1}-s_{3}\right)} \frac{\Gamma\left(\epsilon_{2}-s_{2}\right) \Gamma\left(\epsilon_{3}-s_{4}\right)}{\Gamma\left(\epsilon_{2}+\epsilon_{3}-s_{2}-s_{4}\right)}
\end{aligned}
$$

is a four-fold Mellin-Barnes integral with the contour C specified in the appendix.

In the limit $w_{i t}^{2} \rightarrow 0$ the contours of integration over $\gamma_{i}$ can be moved to the right so the integrals are determined by the residues lying on the real axis to the right of point $\gamma_{i}=-1$. As demonstrated in appendix $\mathrm{D}$, the function $\Lambda$ is regular at small $\gamma_{i}$

$$
\tilde{\Lambda}\left(\gamma_{i}\right)=1+O\left(\gamma_{i}\right)
$$

so the analytic structure of the integral (6.39) is determined by the poles in $\Gamma$-functions and in $[\omega-\aleph(\gamma)]^{-1}$ denominators. The leftmost of such poles are located at $\gamma_{i}=0$ and at $\gamma_{i}^{*}\left(\omega_{i}, g^{2}\right)=$ roots of equation (3.11) which simplifies to

$$
\omega_{i}=\tilde{\aleph}\left(\gamma_{i}, g^{2}\right)=4 g^{2}\left[2 \psi(1)-\psi\left(-\frac{\gamma_{i}}{2}\right)-\psi\left(1+\frac{\gamma_{i}}{2}\right)\right]
$$

in the leading log approximation, see the discussion in section 3.2. 
First, we consider poles at $\gamma_{i}^{*}\left(\omega_{i}, g^{2}\right)$. Taking residues in these poles we obtain

$$
\begin{aligned}
\left\langle\mathcal{F}_{n_{1}}^{j_{1}}\left(x_{1 t}+\frac{w_{1 t}}{2}, x_{1 t}-\frac{w_{1 t}}{2}\right) \mathcal{F}_{n_{2}}^{j_{2}}\left(x_{2 t}+\frac{w_{2 t}}{2}, x_{2 t}-\frac{w_{2 t}}{2}\right) \mathcal{F}_{n_{3}}^{j_{3}}\left(x_{3 t}+\frac{w_{3 t}}{2}, x_{3 t}-\frac{w_{3 t}}{2}\right)\right\rangle \\
=i \frac{64 g^{6}\left(N_{c}^{2}-1\right)}{\pi^{3} x_{12 t}^{2} x_{13 t}^{2} x_{23 t}^{2}} \frac{\omega_{1} \omega_{2} \omega_{3}\left(\frac{s_{12}}{x_{12 t}^{2}}\right)^{\frac{\omega_{1}+\omega_{2}-\omega_{3}}{2}}\left(\frac{s_{13}}{x_{13 t}^{2}}\right)^{\frac{\omega_{1}+\omega_{3}-\omega_{2}}{2}}\left(\frac{s_{23}}{x_{23 t}^{2}}\right)^{\frac{\omega_{2}+\omega_{3}-\omega_{1}}{2}}}{\left(\omega_{1}+\omega_{2}-\omega_{3}\right)\left(\omega_{1}+\omega_{3}-\omega_{2}\right)\left(\omega_{2}+\omega_{3}-\omega_{1}\right)} \tilde{\Lambda}\left(\gamma_{1}^{*}, \gamma_{2}^{*}, \gamma_{3}^{*}\right) \\
\quad \times \prod_{k=1}^{3} \frac{\left(1+\gamma_{k}^{*}\right) 2^{-2 \gamma_{k}^{*}} \Gamma\left(2+\frac{\gamma_{k}^{*}}{2}\right) \Gamma\left(\frac{1}{2}-\frac{\gamma_{k}^{*}}{2}\right)}{\Gamma\left(1-\frac{\gamma_{k}^{*}}{2}\right) \Gamma\left(\frac{3}{2}+\frac{\gamma_{k}^{*}}{2}\right) \gamma_{k}^{* 2} \tilde{\aleph}^{\prime}\left(\gamma_{k}^{*}\right)}\left(\frac{w_{1 t}^{2} x_{23 t}^{2}}{x_{12 t}^{2} x_{13 t}^{2}}\right)^{\frac{\gamma_{1}^{*}}{2}}\left(\frac{w_{2 t}^{2} x_{13 t}^{2}}{x_{12 t}^{2} x_{23 t}^{2}}\right)^{\frac{\gamma_{2}^{*}}{2}}\left(\frac{w_{3 t}^{2} x_{12 t}^{2}}{x_{13 t}^{2} x_{23 t}^{2}}\right)^{\frac{\gamma_{3}^{*}}{2}}
\end{aligned}
$$

Let us present this result in the $\frac{g^{2}}{\omega} \ll 1$ limit. In this limit $\tilde{\aleph}(\gamma) \simeq-\frac{8 g^{2}}{\gamma}$ (see eq. (3.11)) so

$$
\begin{aligned}
& \left\langle\mathcal{F}_{n_{1}}^{j_{1}}\left(x_{1 t}+\frac{w_{1 t}}{2}, x_{1 t}-\frac{w_{1 t}}{2}\right) \mathcal{F}_{n_{2}}^{j_{2}}\left(x_{2 t}+\frac{w_{2 t}}{2}, x_{2 t}-\frac{w_{2 t}}{2}\right) \mathcal{F}_{n_{3}}^{j_{3}}\left(x_{3 t}+\frac{w_{3 t}}{2}, x_{3 t}-\frac{w_{3 t}}{2}\right)\right\rangle \\
& =\frac{i\left(N_{c}^{2}-1\right)}{\pi^{3} x_{12 t}^{2} x_{13 t}^{2} x_{23 t}^{2}} \frac{\omega_{1} \omega_{2} \omega_{3}\left(\frac{s_{12}}{x_{12 t}^{2}}\right)^{\frac{\omega_{1}+\omega_{2}-\omega_{3}}{2}}\left(\frac{s_{13}}{x_{13 t}^{2}}\right)^{\frac{\omega_{1}+\omega_{3}-\omega_{2}}{2}}\left(\frac{s_{23}}{x_{23 t}^{2}}\right)^{\frac{\omega_{2}+\omega_{3}-\omega_{1}}{2}}}{\left(\omega_{1}+\omega_{2}-\omega_{3}\right)\left(\omega_{1}+\omega_{3}-\omega_{2}\right)\left(\omega_{2}+\omega_{3}-\omega_{1}\right)} \\
& \quad \times\left(\frac{w_{1 t}^{2} x_{23 t}^{2}}{x_{12 t}^{2} x_{13 t}^{2}}\right)^{\frac{\gamma_{1}^{*}}{2}}\left(\frac{w_{2 t}^{2} x_{13 t}^{2}}{x_{12 t}^{2} x_{23 t}^{2}}\right)^{\frac{\gamma_{2}^{*}}{2}}\left(\frac{w_{3 t}^{2} x_{12 t}^{2}}{x_{13 t}^{2} x_{23 t}^{2}}\right)^{\frac{\gamma_{3}^{*}}{2}}\left[1+O\left(\frac{g^{2}}{\omega}\right)\right]
\end{aligned}
$$

where $\gamma_{i}^{*}=-\frac{8 g^{2}}{\pi \omega_{i}}$. This result should agree with the first perturbative diagrams calculated in appendix B. Indeed, there are poles in the integral (6.39) at $\gamma_{i}=0$ which give

$$
-i \frac{\left(N_{c}^{2}-1\right)}{\pi^{3} x_{12 t}^{2} x_{13 t}^{2} x_{23 t}^{2}} \frac{\omega_{1} \omega_{2} \omega_{3}\left(\frac{s_{12}}{x_{12 t}^{2}}\right)^{\frac{\omega_{1}+\omega_{2}-\omega_{3}}{2}}\left(\frac{s_{13}}{x_{13 t}^{2}}\right)^{\frac{\omega_{1}+\omega_{3}-\omega_{2}}{2}}\left(\frac{s_{23}}{x_{23 t}^{2}}\right)^{\frac{\omega_{2}+\omega_{3}-\omega_{1}}{2}}}{\left(\omega_{1}+\omega_{2}-\omega_{3}\right)\left(\omega_{1}+\omega_{3}-\omega_{2}\right)\left(\omega_{2}+\omega_{3}-\omega_{1}\right)}
$$

(recall that $\tilde{\Lambda}(0,0,0)=1)$. Similarly to the case of correlator of two light-rays, this term should cancel with the lowest-order diagrams shown in figure 8a. At the tree level the limit $w_{i t} \rightarrow 0$ is trivial so one gets the diagrams in figure 16 which yield eq. (B.16). We see that the result (6.46) cancels with that of eq. (B.16) which justifies our choice of constant $\frac{1}{4}$ in eq. (6.20).

There are also "mixed" poles at $\gamma_{i}=0, \gamma_{j}^{*}, \gamma_{k}^{*}$ and $\gamma_{i}=0, \gamma_{j}=0, \gamma_{k}^{*}$. They should cancel with the contribution of diagrams of the type shown in figure $12 \mathrm{~b}$ and c (recall that our three-dipole approximations are correct starting from the $g^{6}$ diagrams of the figure 9a.)

Thus, the result for all diagrams (figure $7+$ figure 12) is given by eq. (6.44) which translates to the structure constant $C\left(\omega_{i}, g^{2}\right)$ given by eq. (3.14)

$$
F\left(\gamma_{1}^{*}, \gamma_{2}^{*}, \gamma_{3}^{*}\right)=64 g^{6} \tilde{\Lambda}\left(\gamma_{1}^{*}, \gamma_{2}^{*}, \gamma_{3}^{*}\right) \prod_{k=1}^{3} \frac{\left(1+\gamma_{k}^{*}\right) 2^{-2 \gamma_{k}^{*}} \Gamma\left(2+\frac{\gamma_{k}^{*}}{2}\right) \Gamma\left(\frac{1}{2}-\frac{\gamma_{k}^{*}}{2}\right)}{\Gamma\left(1-\frac{\gamma_{k}^{*}}{2}\right) \Gamma\left(\frac{3}{2}+\frac{\gamma_{k}^{*}}{2}\right) \gamma_{k}^{* 2} \tilde{\aleph^{\prime}}\left(\gamma_{k}^{*}\right)}
$$

where $\gamma^{*}$ is the solution of eq. (6.43) and $\tilde{\Lambda}\left(\gamma_{i}\right)=\bar{\Lambda}\left(-2 \epsilon_{i}\right)$ is given by eq. (D.13). It is easy to see that at small $\gamma_{i}^{*}\left(\Leftrightarrow g^{2} \ll \omega_{i} \ll 1\right)$

$$
F\left(\gamma_{1}^{*}, \gamma_{2}^{*}, \gamma_{3}^{*}\right)=1+O\left(\gamma_{i}^{*}\right)=1+O\left(\frac{g^{2}}{\omega_{i}}\right)
$$




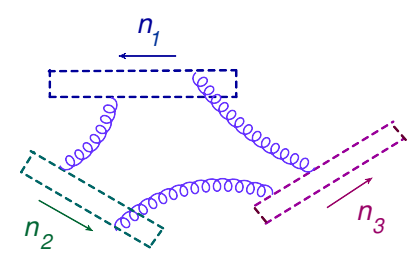

(a)

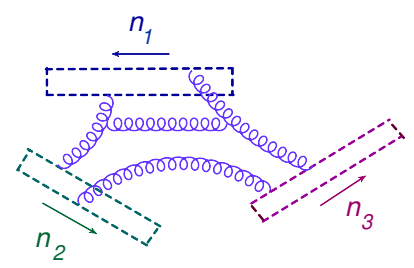

(b)

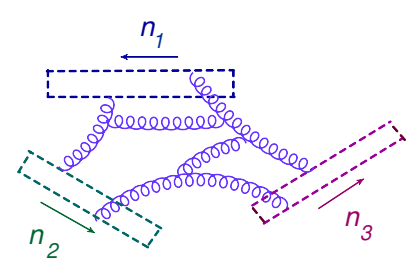

(c)

Figure 12. Typical diagrams for correlator of three Wilson frames in the leading order (a), at the one-loop level (b) and at the two-loop level (c).

\section{Conclusions}

Let us summarize the results of this paper. The correlator of three "forward" light-ray operators (3.7) has the form

$$
\begin{aligned}
& \left\langle S_{1 n_{1}}^{j_{1}}\left(z_{1 t}\right) S_{1 n_{2}}^{j_{2}}\left(z_{2 t}\right) S_{1 n_{3}}^{j_{3}}\left(z_{3 t}\right)\right\rangle \\
& =C\left(\Delta_{i}, j_{i}, g^{2}\right) \frac{\left(-2 n_{1} \cdot n_{2}\right)^{\frac{j_{1}+j_{2}-j_{3}-1}{2}}}{\left|z_{12_{t}}\right|^{\Delta_{1}+\Delta_{2}-\Delta_{3}-1}} \frac{\left(-2 n_{1} \cdot n_{3}\right)^{\frac{j_{1}+j_{3}-j_{2}-1}{2}}}{\left|z_{13_{t}}\right|^{\Delta_{1}+\Delta_{3}-\Delta_{2}-1}} \frac{\left(-2 n_{2} \cdot n_{3}\right)^{\frac{j_{2}+j_{3}-j_{1}-1}{2}}}{\left|z_{23_{t}}\right|^{\Delta_{2}+\Delta_{3}-\Delta_{1}-1}} \mu^{-\gamma_{1}-\gamma_{2}-\gamma_{3}}
\end{aligned}
$$

with the structure constant

$$
C\left(\omega_{i}, g^{2}\right)=\frac{i N_{c}^{2} \omega_{1} \omega_{2} \omega_{3} F\left(\omega_{i}, g^{2}\right)}{\pi^{3}\left(\omega_{1}+\omega_{2}-\omega_{3}\right)\left(\omega_{1}+\omega_{3}-\omega_{2}\right)\left(\omega_{2}+\omega_{3}-\omega_{1}\right)}
$$

At small $\omega_{i}$ the operator $S_{1}^{j}$ can be identified with gluon light-ray operator $\mathcal{F}^{j}$ given by eq. (3.5). In the tree approximation, the correlator of three gluon operators is given by eq. (7.1) with $F=1+O\left(\omega_{i}\right)$ as follows from eq. (B.17). In the BFKL regime $\left(g^{2}, \omega_{i} \ll 1\right.$, $\left.\frac{g^{2}}{\omega_{i}} \sim 1\right)$ the function $F$ has the form (6.47)

$$
F\left(\gamma_{1}^{*}, \gamma_{2}^{*}, \gamma_{3}^{*}\right)=64 g^{6} \tilde{\Lambda}\left(\gamma_{1}^{*}, \gamma_{2}^{*}, \gamma_{3}^{*}\right) \prod_{k=1}^{3} \frac{\left(1+\gamma_{k}^{*}\right) 2^{-2 \gamma_{k}^{*}} \Gamma\left(2+\frac{\gamma_{k}^{*}}{2}\right) \Gamma\left(\frac{1}{2}-\frac{\gamma_{k}^{*}}{2}\right)}{\Gamma\left(1-\frac{\gamma_{k}^{*}}{2}\right) \Gamma\left(\frac{3}{2}+\frac{\gamma_{k}^{*}}{2}\right) \gamma_{k}^{* 2} \tilde{\aleph}^{\prime}\left(\gamma_{k}^{*}\right)}
$$

where $\gamma^{*}$ is the solution of eq. (6.43).

Let us now discuss main features of the result (7.3). First, note that since $\gamma^{*}$ is real, in our LLA approximation the constant $F$ is real since all the functions in the r.h.s. of eq. (6.47) are real. Indeed, for $\Gamma$-functions it is trivial and for $\tilde{\Lambda}\left(\gamma_{1}^{*}, \gamma_{2}^{*}, \gamma_{3}^{*}\right)$ it follows from the explicit expression (D.14). This is in accordance with the fact that physical schannel imaginary part of the amplitude (6.1) vanishes in our approximation. Indeed, it would correspond to "cut" diagram of figure 13a type and cut propagator connecting two infinite Wilson lines in $n_{2}$ and $n_{3}$ directions vanishes (see the last line in the eq. (A.10) in appendix A). The imaginary part comes from the next terms of the expansion in powers of $g^{2}$ and $\omega$. The imaginary part $\sim \omega$ is given by the second term in the square brackets in the r.h.s. of tree-level expression (B.16). As to imaginary part $\sim g^{2}$, it comes from the diagrams of the type shown in figure 13b. These diagrams were calculated in the $n_{2} \rightarrow n_{3}$ limit in refs. [26, 27] and the result is given by eq. (C.12) or eq. (C.14) for small $g^{2} / \omega$. Note that the result (C.11) has the same structure as eq. (7.3). 


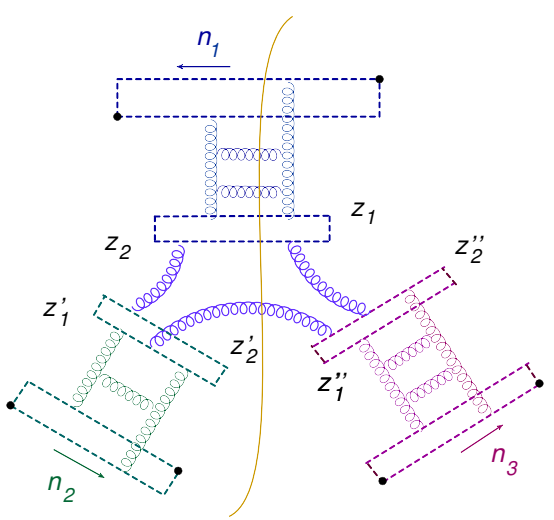

(a)

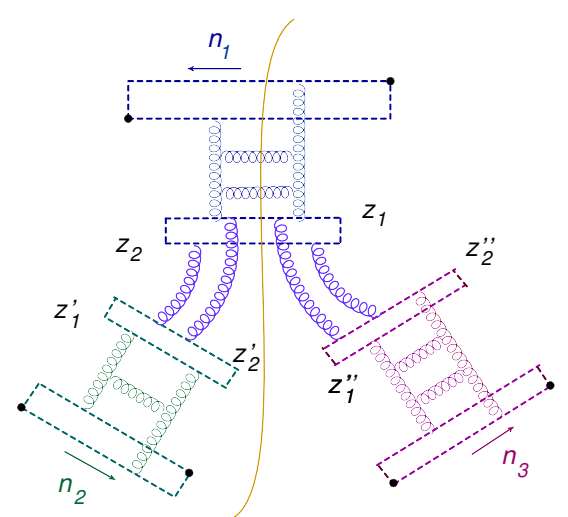

(b)

Figure 13. Typical "cut diagrams" for the correlator of three Wilson frames.

We saw that the structure constant has poles at $\omega_{i}=\omega_{j}+\omega_{k}$ reflecting boost invariance at $n_{j} \rightarrow n_{k}$. An interesting question is what are singularities in the function $F\left(\omega_{i}, g^{2}\right)$ apart from obvious singular point $\omega_{i}=0$, for example like $\frac{1}{\omega_{1}-\omega_{2}}$. It is worthwhile to note that such terms appeared in the intermediate steps in the calculation of $\Lambda$ at small $\frac{g^{2}}{\omega_{i}}$, for example the term $J_{6}$ contains $\frac{1}{\epsilon_{1}-\epsilon_{2}} \rightarrow \frac{g^{2} \omega_{1} \omega_{2}}{\omega_{1}-\omega_{2}}$, see eq. (D.32). There were also other singularities which all canceled in the final result (D.34) so it suggests that the function $F$ is finite at $\omega_{i} \neq 0$.

In conclusion, let us discuss the applicability of our results to QCD correlators of gluon light-ray operators. In the leading log approximation considered here the formulas for correlators will be the same as in $\mathcal{N}=4$ case since running of the coupling constant is beyond the LLA approximation, and since the contribution of scalar and gluino operators is negligible at small $\omega$. At the NLO level, in $\mathcal{N}=4$ case we expect only corrections to structure constant of the type of eq. (7.3), but in QCD the functional form of two- and three-point correlators may change. An example of such change is the modification of the formula (5.14) for the $\gamma^{*} \gamma^{*}$ amplitude in QCD calculated at the NLO BFKL level in refs. [42, 43]. It would be interesting to write down such modifications for the correlators of gluon-light-ray operators at the NLO BFKL level in QCD.

The author is grateful to V. Kazakov, G. Korchemsky, and E. Sobko for valuable discussions. This work is supported by contract DE-AC05-06OR23177 under which the Jefferson Science Associates, LLC operate the Thomas Jefferson National Accelerator Facility, and by the grant DE-FG02-97ER41028.

\section{A BFKL kernel in the triple Regge limit}

In this section I will demonstrate how the BFKL kernel comes out of the conventional momentum-space calculation in the triple Regge limit. Let us again consider the first diagram in figure 9. Our LLA approximation (6.19) in the momentum space reads

$$
\alpha \beta s_{12} \gg m_{\perp}^{2}, \quad \alpha \gamma s_{13} \gg m_{\perp}^{2}, \quad \beta \gamma s_{23} \gg m_{\perp}^{2}
$$




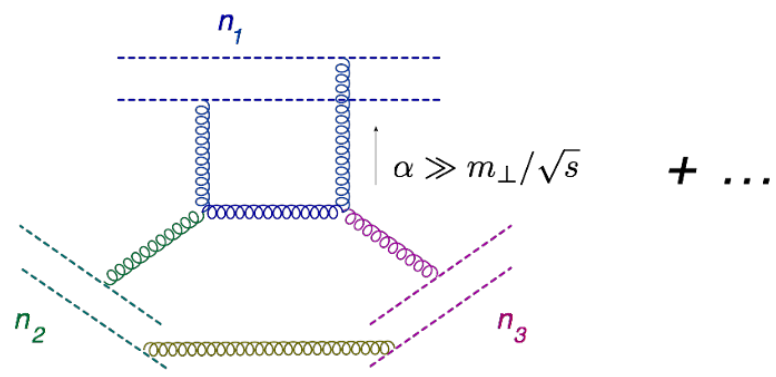

Figure 14. Leading diagrams with logarithmic integrals over $\alpha$.

If all $s_{i j}$ are of the same order, this translates to $\alpha \gg m_{\perp} \sqrt{\frac{s_{23}}{s_{12} s_{13}}}$ etc. ${ }^{14}$ Suppose now that we already performed integrals over $\beta$ and $\gamma$ which results in logs multiplied by (conformal) dipoles and we would like to consider the last integral over $\alpha$ coming from the diagrams of figure 14 type.

To avoid cluttering of formulas, we will disregard the bottom gluon connecting Wilson lines parallel to $n_{2}$ and $n_{3}$. Indeed, the corresponding factor

$$
\left\langle V\left(z_{3 t} e_{t}+\breve{z}_{3} \breve{e}\right) W\left(z_{5 t} e_{t}+\check{z}_{5} \check{e}\right)\right\rangle=i \alpha_{s} t^{a} \otimes t^{a} \ln \left[z_{35 t}^{2}+\left(\breve{z}_{3}+\check{z}_{5}\right)^{2}\right]
$$

(plus permutations) simply multiplies contributions of diagrams in figure 15 and has nothing to do with logarithm coming out of $\alpha$ integration.

To simplify our formulas, let us calculate the "cut diagram" shown in figure 15 . It can be represented by a functional integral over double set of variables: fields to the left and to the right of the cut which coincide at $t=\infty .{ }^{15}$ We get

$$
\begin{aligned}
\langle\operatorname{Tr} & \left.\left\{\tilde{U}^{\dagger}\left(x_{1}\right) U\left(x_{1}\right) U^{\dagger}\left(x_{2}\right) \tilde{U}\left(x_{2}\right)\right\} \tilde{V}^{\dagger}\left(x_{3}\right) W\left(x_{4}\right)\right\rangle \\
= & g_{\mathrm{YM}}^{6} N_{c} t^{a} \otimes t^{a} \int d^{4} k_{1} d^{4} k_{1}^{\prime} d^{4} k_{2} d^{4} k_{2}^{\prime} \delta^{4}\left(k_{2}+k_{1}-k_{2}^{\prime}-k_{1}^{\prime}\right) \delta\left(\left(k_{2}+k_{1}\right)^{2}\right) \theta\left(k_{2}+k_{1}\right)_{0} \\
& \times \delta\left(k_{2} \cdot n_{2}\right) \delta\left(k_{1} \cdot n_{1}\right) \delta\left(k_{2}^{\prime} \cdot n_{3}\right) \delta\left(k_{1}^{\prime} \cdot n_{1}\right) \frac{L^{\lambda}\left(k_{2}, k_{1}\right) L_{\lambda}\left(k_{2}^{\prime}, k_{1}^{\prime}\right)}{k_{1}^{2} k_{2}^{2} k_{1}^{\prime 2} k_{2}^{\prime 2}} e^{i k_{2 t} x_{3 t}+i \breve{k}_{2} \breve{x}_{3}} \\
& \times\left(e^{i k_{1 t} x_{1 t}+i \tilde{k}_{1} \tilde{x}_{1}}-e^{i k_{1 t} x_{2 t}+i \tilde{k}_{1} \tilde{x}_{2}}\right) e^{-i k_{2 t}^{\prime} x_{4 t}-i \check{k}_{2}^{\prime} \check{x}_{4}}\left(e^{-i k_{1 t}^{\prime} x_{1 t}-i \tilde{k}_{1}^{\prime} \tilde{x}_{1}}-e^{-i k_{1 t}^{\prime} x_{2 t}-i \tilde{k}_{1}^{\prime} \tilde{x}_{2}}\right)
\end{aligned}
$$

where we denoted Wilson lines to the left of the cut by tilde. Here we use space-saving notations $d^{n} k \equiv \frac{d^{n} k}{(2 \pi)^{n}}$ and $\delta^{(n)}(k) \equiv(2 \pi)^{n} \delta^{(n)}(k)$. The Lipatov vertex of gluon emission can be taken e.g. from ref. [47]

$$
\begin{aligned}
& \frac{2}{s_{12}} L\left(k_{2}, k_{1}\right)=\left(k_{1}-k_{2}\right)-\left[2 \frac{k_{1 n_{2}}}{n_{12}}+\frac{k_{1}^{2}}{k_{2 n_{1}}}\right] n_{1}+\left[2 \frac{k_{2 n_{1}}}{n_{12}}+\frac{k_{2}^{2}}{k_{1 n_{2}}}\right] n_{2} \\
& \frac{2}{s_{13}} L\left(k_{2}^{\prime}, k_{1}^{\prime}\right)=\left(k_{1}^{\prime}-k_{2}^{\prime}\right)-\left[2 \frac{k_{1 n_{3}}^{\prime}}{n_{13}}+\frac{k_{1}^{\prime 2}}{k_{2 n_{1}}^{\prime}}\right] n_{1}+\left[2 \frac{k_{2 n_{1}}^{\prime}}{n_{13}}+\frac{k_{2}^{\prime 2}}{k_{1 n_{3}}^{\prime}}\right] n_{3}
\end{aligned}
$$

\footnotetext{
${ }^{14}$ Indeed, if $\alpha \beta s_{12}=\lambda_{3} m_{\perp}^{2}, \alpha \gamma s_{13}=\lambda_{2} m_{\perp}^{2}, \beta \gamma s_{13}=\lambda_{1} m_{\perp}^{2}$ we get $\alpha=\sqrt{\frac{\lambda_{2} \lambda_{3}}{\lambda_{1}}} m_{\perp} \sqrt{\frac{s_{23}}{s_{12} s_{13}}}$ and therefore $\alpha \gg m_{\perp} \sqrt{\frac{s_{23}}{s_{12} s_{13}}}$ if all $\lambda$ 's are large and of the same order.

${ }^{15}$ If $n_{2}=n_{3}$ and the corresponding dipoles are the same, the double functional integral for the cut diagram gives the imaginary part of the non-cut diagram, see e.g. the discussion in refs. [44-46].
} 


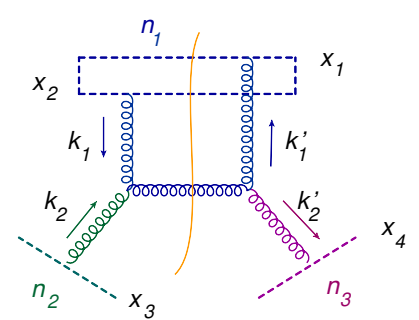

(a)

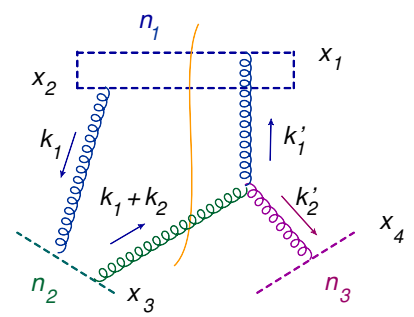

(b)

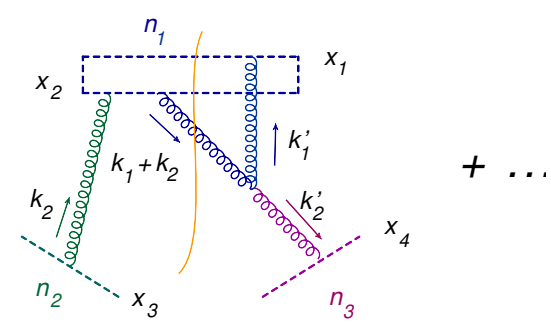

(c)

Figure 15. First rung of the BFKL evolution in the triple Regge limit.

Rewriting these formulas in terms of triple Sudakov variables (6.2) and taking into account $\delta$-functions in the r.h.s. of eq. (A.2) we obtain

$$
\begin{aligned}
& \frac{2}{s_{12}} L\left(k_{1}, k_{2}\right)=\left(k_{1}-k_{2}\right)_{t}+ \\
& \quad+n_{1}\left[-\alpha_{1}-\alpha_{2}-\frac{k_{1}^{2}}{k_{2 n_{1}}}-2 \gamma_{1} \frac{n_{23}}{n_{12}}\right]+n_{2}\left[\beta_{1}+\beta_{2}+\frac{k_{2}^{2}}{k_{1 n_{2}}}+2 \gamma_{2} \frac{n_{13}}{n_{12}}\right]+\left(\gamma_{1}-\gamma_{2}\right) n_{3} \\
& \frac{2}{s_{13}} L\left(k_{1}^{\prime}, k_{2}^{\prime}\right)=\left(k_{1}^{\prime}-k_{2}^{\prime}\right)_{t} \\
& \quad+n_{1}\left[-\alpha_{1}^{\prime}-\alpha_{2}^{\prime}-\frac{k_{1}^{\prime 2}}{k_{2 n_{1}}}-2 \beta_{1}^{\prime} \frac{n_{23}}{n_{13}}\right]+\left(\beta_{1}^{\prime}-\beta_{2}^{\prime}\right) n_{2}+n_{3}\left[\gamma_{1}^{\prime}+\gamma_{2}^{\prime}+\frac{k_{2}^{\prime 2}}{k_{1 n_{3}}^{\prime}}+2 \beta_{2}^{\prime} \frac{n_{12}}{n_{13}}\right]
\end{aligned}
$$

In our LLA approximation $\alpha_{1} \gg m \sqrt{\frac{s_{23}}{s_{12} s_{13}}}$ and $\beta_{1} \sim \beta_{1}^{\prime} \sim \beta_{2}^{\prime} \sim m \sqrt{\frac{s_{13}}{s_{12} s_{23}}}, \alpha_{2} \sim \alpha_{2}^{\prime} \sim$ $m \sqrt{\frac{s_{23}}{s_{12} s_{13}}}, \gamma_{1} \sim \gamma_{2} \sim m \sqrt{\frac{s_{12}}{s_{13} s_{23}}}$. Moreover, from $\delta$-function $\delta\left(\left(k_{2}+k_{1}\right)^{2}\right) \theta\left(k_{2}+k_{1}\right)_{0}$ we see that $\xi_{2}=\beta_{2}-\alpha_{2} \frac{s_{13}}{s_{23}}=\frac{s_{13}}{s_{12}}\left(\gamma_{2}^{\prime}-\alpha_{2}^{\prime} \frac{s_{12}}{s_{23}}\right) \ll \frac{m}{\sqrt{s}}$. Using these approximations, one obtains after some algebra

$$
\begin{aligned}
& \frac{2}{s_{12} s_{13}} L\left(k_{2}, k_{1}\right) L\left(k_{2}^{\prime}, k_{1}^{\prime}\right)=-\left(k_{1}-k_{1}^{\prime}\right)_{t}^{2}-\frac{s_{12} s_{23}}{s_{13}}\left(\beta_{1}-\beta_{1}^{\prime}\right)^{2} \\
& \quad+\frac{\left(k_{1 t}^{2}+\frac{s_{12} s_{23}}{s_{13}} \beta_{1}^{2}\right)\left(k_{2 t}^{\prime 2}+\frac{s_{12} s_{23}}{s_{13}} \beta_{2}^{\prime 2}\right)}{\left(k_{1}+k_{2}\right)_{t}^{2}+\frac{s_{12} s_{23}}{s_{13}}\left(\beta_{1}+\beta_{2}\right)^{2}}+\frac{\left(k_{2 t}^{2}+\frac{s_{12} s_{23}}{s_{13}} \beta_{2}^{2}\right)\left(k_{1 t}^{\prime}+\frac{s_{12} s_{23}}{s_{13}} \beta_{1}^{\prime 2}\right)}{\left(k_{1}+k_{2}\right)_{t}^{2}+\frac{s_{12} s_{23}}{s_{13}}\left(\beta_{1}+\beta_{2}\right)^{2}}+O\left(\frac{k_{t}^{2}}{\alpha_{1} s}\right)
\end{aligned}
$$

which is a "real part" of the BFKL kernel.

The amplitude can be rewtitten as

$$
\begin{aligned}
& \left\langle\operatorname{Tr}\left\{\tilde{U}^{\dagger}\left(x_{1}\right) U\left(x_{1}\right) U^{\dagger}\left(x_{2}\right) \tilde{U}\left(x_{2}\right)\right\} \tilde{V}^{\dagger}\left(x_{3}\right) W\left(x_{4}\right)\right\rangle \\
& =\frac{g_{\mathrm{YM}}^{6} N_{c}}{4 \pi} t^{a} \otimes t^{a} \int_{0}^{\infty} \frac{d \alpha_{1}}{\alpha_{1}} \int d k_{1 t} d k_{1 t}^{\prime} d k_{2 t} \succsim k_{2 t}^{\prime} \delta\left(k_{1}+k_{2}-k_{1}^{\prime}-k_{2}^{\prime}\right)_{t} \\
& \times \int đ \tilde{k}_{1} む \tilde{k}_{1}^{\prime} む \breve{k}_{2} \succsim \check{k}_{2}^{\prime} \delta\left(\tilde{k}_{1}-\breve{k}_{2}-\tilde{k}_{1}^{\prime}+\check{k}_{2}^{\prime}\right) \\
& \times\left(e^{i k_{1 t} x_{1 t}+i \tilde{k}_{1} \tilde{x}_{1}}-e^{i k_{1 t} x_{2 t}+i \tilde{k}_{1} \tilde{x}_{2}}\right)\left(e^{-i\left(k_{1}^{\prime}, x_{1}\right)_{t}}-e^{-i\left(k_{1}^{\prime}, x_{2}\right) t}\right) e^{i k_{2 t} x_{3 t}+i \breve{k}_{2} \breve{x}_{3}} e^{-i k_{2 t}^{\prime} x_{4 t}-i \breve{k}_{2}^{\prime} \breve{x}_{4}} \\
& \times \frac{-\left(k_{1}-k_{1}^{\prime}\right)_{t}^{2}-\left(\tilde{k}_{1}-\tilde{k}_{1}^{\prime}\right)^{2}+\frac{\left(k_{1 t}^{2}+\tilde{k}_{1}^{2}\right)\left(k_{2 t}^{\prime 2}+\tilde{k}_{2}^{2}\right)+\left(k_{2 t}^{2}+\tilde{k}_{2}^{2}\right)\left(k_{1 t}^{\prime 2}+{\tilde{k^{\prime}}}_{1}^{2}\right)}{\left(k_{1}+k_{1}^{\prime}\right)_{t}^{2}+\left(\tilde{k}_{1}+\tilde{k}_{1}^{\prime}\right)^{2}}}{\left(k_{1 t}^{2}+\tilde{k}_{1}^{2}\right)\left({k^{\prime}}_{1 t}^{2}+{\tilde{k^{\prime}}}_{1}^{2}\right)\left(k_{2 t}^{2}+\breve{k}_{2}^{2}\right)\left({k^{\prime}}_{2 t}^{2}+{\check{k^{\prime}}}_{2}^{2}\right)}
\end{aligned}
$$


Note that at $n_{2}=n_{3}$ this formula reduces to the first rung of the BFKL ladder for dipoledipole cross section [47]

In this form it coincides with the first iteration of the evolution equation for color dipoles. Let us demonstrate this for the simple term in the BFKL kernel $2 k_{1 t} k_{1 t}^{\prime}+2 \tilde{k}_{1} \tilde{k}_{1}^{\prime}$. Performing momentum integrals one obtains

$$
\begin{aligned}
& \frac{g_{\mathrm{YM}}^{6} N_{c}}{4 \pi} t^{a} \otimes t^{a} \int_{0}^{\infty} \frac{d \alpha_{1}}{\alpha_{1}} \int d k_{1 t} d k_{1 t}^{\prime} d k_{2 t} d k_{2 t}^{\prime} \delta\left(k_{1}+k_{2}-k_{1}^{\prime}-k_{2}^{\prime}\right)_{t} \\
& \quad \times \int d \tilde{k}_{1} d \tilde{k}_{1}^{\prime} đ \breve{k}_{2} d \check{k}_{2}^{\prime} \delta\left(\tilde{k}_{1}-\breve{k}_{2}-\tilde{k}_{1}^{\prime}+\check{k}_{2}^{\prime}\right) \frac{2 k_{1 t} k_{1 t}^{\prime}+2 \tilde{k}_{1} \tilde{k}_{1}^{\prime}}{\left(k_{1 t}^{2}+\tilde{k}_{1}^{2}\right)\left(k_{1 t}^{\prime 2}+\tilde{k}_{1}^{\prime 2}\right)\left(k_{2 t}^{2}+\breve{k}_{2}^{2}\right)\left(k^{\prime 2}{ }_{2 t}+\check{k}_{2}^{\prime 2}\right)} \\
& \quad \times\left(e^{i k_{1 t} x_{1 t}+i \tilde{k}_{1} \tilde{x}_{1}}-e^{i k_{1 t} x_{2 t}+i \tilde{k}_{1} \tilde{x}_{2}}\right)\left(e^{-i\left(k_{1}^{\prime}, x_{1}\right)_{t}}-e^{-i\left(k_{1}^{\prime}, x_{2}\right)_{t}}\right) e^{i k_{2 t} x_{3 t}+i \breve{k}_{2} \breve{x}_{3}} e^{-i k_{2 t}^{\prime} x_{4 t}-i \check{k}_{2}^{\prime} \check{x}_{4}} \\
& =\frac{\alpha_{s}^{3} N_{c}}{2 \pi^{2}} t^{a} \otimes t^{a} \int_{0}^{\infty} \frac{d \alpha_{1}}{\alpha_{1}} \\
& \quad \times \int d^{2} z_{\perp} \frac{\left(x_{1}-x_{2}\right)_{\perp}^{2}}{\left(x_{1}-z\right)^{2}\left(x_{2}-z\right)^{2}} \ln \left[\left(x_{3 t}-z_{t}\right)^{2}+\left(\breve{x}_{3}+\tilde{z}\right)^{2}\right] \ln \left[\left(x_{4 t}-z_{t}\right)^{2}+\left(\check{x}_{4}+\tilde{z}\right)^{2}\right]
\end{aligned}
$$

where $z_{\perp} \equiv z_{t}, \tilde{z}$.

On the other hand, the (linearized) evolution equation for color dipoles (in the double functional integral formalism) reads [41, 48]

$$
\begin{aligned}
& \frac{d}{d Y} \operatorname{Tr}\left\{\tilde{U}^{\dagger}\left(x_{1}\right) U\left(x_{1}\right) U^{\dagger}\left(x_{2}\right) \tilde{U}\left(x_{2}\right)\right\} \\
& =\frac{\alpha_{s} N_{c}}{2 \pi} \int d^{2} z_{\perp} \frac{\left(x_{1}-x_{2}\right)_{\perp}^{2}}{\left(x_{1}-z\right)_{\perp}^{2}\left(x_{2}-z\right)_{\perp}^{2}}\left[\operatorname{Tr}\left\{\tilde{U}^{\dagger}\left(x_{1}\right) U\left(x_{1}\right) U^{\dagger}(z) \tilde{U}(z)\right\}\right. \\
& \left.\quad+\operatorname{Tr}\left\{\tilde{U}^{\dagger}(z) U(z) U^{\dagger}\left(x_{2}\right) \tilde{U}\left(x_{2}\right)\right\}-\operatorname{Tr}\left\{\tilde{U}^{\dagger}\left(x_{1}\right) U\left(x_{1}\right) U^{\dagger}\left(x_{2}\right) \tilde{U}\left(x_{2}\right)\right\}\right]
\end{aligned}
$$

where $Y \equiv \ln \alpha$. The term (A.7) comes from the correlator

$$
\begin{aligned}
& \frac{\alpha_{s} N_{c}}{2 \pi} \int_{0}^{\infty} \frac{d \alpha}{\alpha} \int d^{2} z_{\perp} \frac{-2\left(x_{1}-z, x_{2}-z\right)_{\perp}}{\left(x_{1}-z\right)_{\perp}^{2}\left(x_{2}-z\right)_{\perp}^{2}} \\
& \times\left\langle\operatorname{Tr}\left\{U^{\dagger}(z) \tilde{U}(z)+\tilde{U}^{\dagger}(z) U(z)\right\} \tilde{V}^{\dagger}\left(x_{3}\right) W\left(x_{4}\right)\right\rangle
\end{aligned}
$$

Using the tree-level correlators of Wilson lines

$$
\begin{aligned}
\left\langle\tilde{U}\left(z_{t} e_{t}+\tilde{z} \tilde{e}\right) \tilde{V}\left(x_{3 t} e_{t}+\breve{x}_{3} \breve{e}\right)\right\rangle & =i \alpha_{s} t^{a} \otimes t^{a} \ln \left[\left(z-x_{3}\right)_{t}^{2}+\left(\tilde{z}+\breve{x}_{3}\right)^{2}\right], \\
\left\langle U\left(z_{t} e_{t}+\tilde{z} \tilde{e}\right) W\left(x_{4 t} e_{t}+\check{z}_{4} \check{e}\right)\right\rangle & =-i \alpha_{s} t^{a} \otimes t^{a} \ln \left[\left(z-x_{4}\right)_{t}^{2}+\left(\tilde{z}+\check{x}_{4}\right)^{2}\right], \\
\left\langle\tilde{U}\left(z_{t} e_{t}+\tilde{z} \tilde{e}\right) W\left(x_{4 t} e_{t}+\check{x}_{4} \check{e}\right)\right\rangle & =\left\langle U\left(z_{t} e_{t}+\tilde{z} \tilde{e}\right) \tilde{V}\left(x_{3 t} e_{t}+\check{x}_{3} \check{e}\right)\right\rangle=0
\end{aligned}
$$

it is easy to see that eq. (A.9) coincides with the r.h.s. of eq. (A.7). Similarly, one can check that other terms in the BFKL kernel correspond to linear part of the evolution equation for color dipoles (A.8), see e.g. the book [49]. 


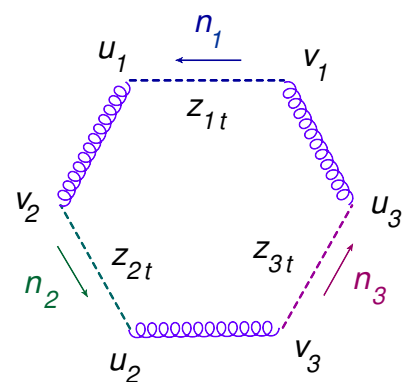

(a)

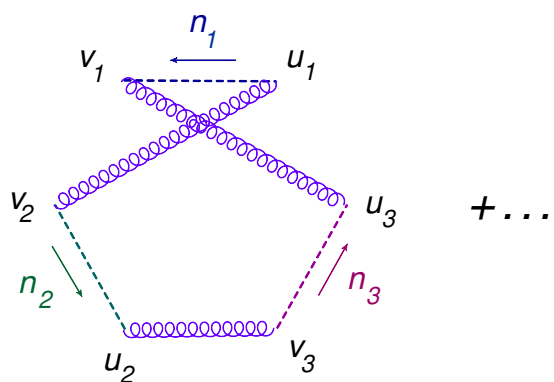

(b)

Figure 16. Tree-level correlator of three twist-two gluon LR operators.

\section{B Correlator of three twist-2 LR operators in the tree approximation}

First, we calculate the correlator of two light-ray gluon operators. Using bare propagator

$$
\begin{aligned}
\pi^{2}\left\langle F_{n_{1}}{ }^{\mu}\left(u_{1} n_{1}+x_{1_{\perp}}\right) F_{n_{2}}{ }^{\nu}\left(u_{2} n_{2}+x_{2_{\perp}}\right)\right\rangle \\
=-\frac{g^{\mu \nu} \delta^{a b} s_{12} x_{12_{\perp}}^{2}}{2\left(u_{1} u_{2} s_{12}+x_{12_{\perp}}^{2}+i \epsilon\right)^{3}}-\frac{\delta^{a b} n_{2}^{\mu} n_{1}^{\nu}}{\left(u_{1} u_{2} s_{12}+x_{12}^{2}+i \epsilon\right)^{2}} \\
\quad-\frac{s_{12} \delta^{a b}}{\left(u_{1} u_{2} s_{12}+x_{12_{\perp}}^{2}+i \epsilon\right)^{3}}\left[u_{2} x_{12}^{\mu} n_{2}^{\nu}-u_{1} n_{1}^{\mu} x_{12}^{\nu}-x_{12}^{\mu} x_{12}^{\nu}+u_{1} u_{2}\left(n_{1}^{\mu} n_{2}^{\nu}-n_{2}^{\mu} n_{1}^{\nu}\right)\right]
\end{aligned}
$$

after simple integration we get the tree-level correlator in the form ${ }^{16}$

$$
\frac{1}{N_{c}^{2}-1}\left\langle\mathcal{F}_{n_{1}}^{j}\left(x_{1_{\perp}}\right) \mathcal{F}_{n_{2}}^{j^{\prime}}\left(x_{2_{\perp}}\right)\right\rangle=-\frac{i s^{4}}{2 \pi} \delta\left(\omega-\omega^{\prime}\right) \frac{1-e^{i \pi \omega}}{\sin \pi \omega} \frac{\omega(1+\omega)}{\left(x_{12_{\perp}}^{2}\right)^{2+\omega} s^{-\omega}} \frac{\Gamma(2+\omega) \Gamma(4+\omega)}{\Gamma(4+2 \omega)}
$$

which agrees with eq. (5.40) in the limit $\omega \rightarrow 0$

$$
\frac{1}{N_{c}^{2}-1}\left\langle\mathcal{F}_{n_{1}}^{j}\left(x_{1_{\perp}}\right) \mathcal{F}_{n_{2}}^{j^{\prime}}\left(x_{2_{\perp}}\right)\right\rangle \simeq-\delta\left(j-j^{\prime}\right) \frac{\omega}{2 \pi x_{12_{\perp}}^{4}} \frac{s^{\omega}}{x_{12_{\perp}}^{2 \omega}}
$$

as discussed in the end of section 5.2.

Next, consider diagrams in figure 16 representing the 3-point correlator of gluon lightray operators in the leading perturbative order.

After some algebra one obtains the result for the first diagram in figure 16a in the form

$$
\begin{aligned}
& \begin{array}{c}
\left\langle F_{n_{1}}^{a \mu}\left(u_{1} n_{1}+z_{1 t}\right) F_{n_{2}}^{b \nu}\left(v_{2} n_{2}+z_{2 t}\right)\right\rangle\left\langle F_{n_{1} \mu}^{a}\left(v_{1} n_{1}+z_{1 t}\right) F_{n_{3}}^{c \lambda}\left(u_{3} n_{3}+z_{3 t}\right)\right\rangle \\
\times\left\langle F_{n_{2} \nu}^{b}\left(u_{2} n_{2}+z_{2 t}\right) F_{n_{3} \lambda}^{c}\left(v_{3} n_{3}+z_{3 t}\right)\right\rangle
\end{array} \\
& =\frac{N_{c}^{2}-1}{4 \pi^{6} s_{12} s_{23} s_{13}} \frac{z_{12_{t}}^{2} z_{13_{t}}^{2} z_{23_{t}}^{2}}{\left(u_{1} v_{2} s_{12}+z_{12_{t}}^{2}+i \epsilon\right)^{3}\left(v_{1} u_{3} s_{13}+z_{13_{t}}^{2}+i \epsilon\right)^{3}\left(u_{2} v_{3} s_{23}+z_{23_{t}}^{2}+i \epsilon\right)^{3}}
\end{aligned}
$$

\footnotetext{
${ }^{16}$ As we discussed above, $\delta\left(j-j^{\prime}\right)$ actually means "analytic continuation" of $\delta\left(\nu-\nu^{\prime}\right)$ for $j=\frac{1}{2}+i \nu$, $j^{\prime}=\frac{1}{2}+i \nu^{\prime}$.
} 
where $s_{12} \equiv-2 n_{1} \cdot n_{2}$ etc. Adding the diagrams with permutations we obtain

$$
\begin{aligned}
& \int_{-\infty}^{\infty} d u_{1} d v_{1} d u_{2} d v_{2} d u_{3} d v_{3} \theta\left(u_{1}-v_{1}\right) \theta\left(u_{2}-v_{2}\right) \theta\left(u_{3}-v_{3}\right) \\
& \times\left(u_{1}-v_{1}\right)^{-\omega_{1}}\left(u_{2}-v_{2}\right)^{-\omega_{2}}\left(u_{3}-v_{3}\right)^{-\omega_{3}}\left\langle F_{n_{1}}^{a \mu}\left(u_{1} n_{1}+z_{1 t}\right) F_{n_{1} \mu}^{a}\left(v_{1} n_{1}+z_{1 t}\right)\right. \\
&\left.\times F_{n_{2} \nu}^{b \nu}\left(v_{2} n_{2}+z_{2 t}\right) F_{n_{2} \nu}^{b}\left(u_{2} n_{2}+z_{2 t}\right) F_{n_{3}}^{c \lambda}\left(u_{3} n_{3}+z_{3 t}\right) F_{n_{3} \lambda}^{c}\left(v_{3} n_{3}+z_{3 t}\right)\right\rangle \\
&=\frac{N_{c}^{2}-1}{4 \pi^{6} s_{12} s_{23} s_{13}} \int_{-\infty}^{\infty} d u_{1} d v_{1} d u_{2} d v_{2} d u_{3} d v_{3}\left[\theta\left(u_{1}-v_{1}\right)\left(u_{1}-v_{1}\right)^{-\omega_{1}}+u_{1} \leftrightarrow v_{1}\right] \\
& \quad \times\left[\theta\left(u_{2}-v_{2}\right)\left(u_{2}-v_{2}\right)^{-\omega_{2}}+u_{2} \leftrightarrow v_{2}\right]\left[\theta\left(u_{3}-v_{3}\right)\left(u_{3}-v_{3}\right)^{-\omega_{3}}+u_{3} \leftrightarrow v_{3}\right] \\
& \quad \frac{z_{12 t}^{2} z_{13_{t}}^{2} z_{23_{t}}^{2}}{\times\left(u_{1} v_{2} s_{12}+z_{12 t}^{2}+i \epsilon\right)^{3}\left(v_{1} u_{3} s_{13}+z_{13 t}^{2}+i \epsilon\right)^{3}\left(u_{2} v_{3} s_{23}+z_{23_{t}}^{2}+i \epsilon\right)^{3}}
\end{aligned}
$$

The integration over light-ray variables $u_{i}, v_{i}$ is done with the help of two formulas:

$$
\begin{aligned}
& \int_{-\infty}^{\infty} d t_{1} d t_{2} d t_{3} \int_{0}^{\infty} d r_{1} d r_{2} d r_{3} \frac{1}{\left[\left(r_{1}+t_{1}\right)\left(r_{2}+t_{2}\right)+a_{12}^{2}+i \epsilon\right]} \\
& \quad \times\left\{\frac{r_{1}^{-\omega_{1}} r_{2}^{-\omega_{2}} r_{3}^{-\omega_{3}}}{\left[t_{1}\left(r_{3}+t_{3}\right)+a_{13}^{2}+i \epsilon\right]\left(t_{2} t_{3}+a_{23}^{2}+i \epsilon\right)}+\frac{r_{1}^{-\omega_{1}} r_{2}^{-\omega_{2}} r_{3}^{-\omega_{3}}}{\left(t_{1} t_{3}+a_{13}^{2}+i \epsilon\right)\left[t_{2}\left(r_{3}+t_{3}\right)+a_{23}^{2}+i \epsilon\right]}\right\} \\
& =-4 \sin \frac{\pi \omega_{3}}{2} \sin \frac{\pi}{2}\left(\omega_{1}+\omega_{2}-\omega_{3}\right) \cos \frac{\pi}{2}\left(\omega_{1}-\omega_{2}\right) \Gamma\left(1-\omega_{1}\right) \Gamma\left(1-\omega_{2}\right) \Gamma\left(1-\omega_{3}\right) \\
& \quad \times \Gamma^{2}\left(\frac{\omega_{1}+\omega_{2}-\omega_{3}}{2}\right) \Gamma^{2}\left(\frac{\omega_{2}+\omega_{3}-\omega_{1}}{2}\right) \Gamma^{2}\left(\frac{\omega_{1}+\omega_{3}-\omega_{2}}{2}\right) a_{12}^{\omega_{3}-\omega_{1}-\omega_{2}} a_{13}^{\omega_{2}-\omega_{1}-\omega_{3}} a_{23}^{\omega_{1}-\omega_{2}-\omega_{3}}
\end{aligned}
$$

and

$$
\begin{aligned}
& \int_{-\infty}^{\infty} d t_{1} d t_{2} d t_{3} \int_{0}^{\infty} d r_{1} d r_{2} d r_{3} \\
& \times\left[\frac{r_{1}^{-\omega_{1}} r_{2}^{-\omega_{2}} r_{3}^{-\omega_{3}}}{\left[\left(r_{1}+t_{1}\right) t_{2}+a_{12}+i \epsilon\right]\left[t_{1}\left(r_{3}+t_{3}\right)+a_{13}+i \epsilon\right]\left[\left(r_{2}+t_{2}\right) t_{3}+a_{23}+i \epsilon\right]}\right. \\
& \left.\quad+\frac{r_{1}^{-\omega_{1}} r_{2}^{-\omega_{2}} r_{3}^{-\omega_{3}}}{\left[t_{1}\left(r_{2}+t_{2}\right)+a_{12}+i \epsilon\right]\left[\left(r_{1}+t_{1}\right) t_{3}+a_{13}+i \epsilon\right]\left[t_{2}\left(r_{3}+t_{3}\right)+a_{23}+i \epsilon\right]}\right] \\
& =\Gamma\left(1-\omega_{1}\right) \Gamma\left(1-\omega_{2}\right) \Gamma\left(1-\omega_{3}\right) \Gamma^{2}\left(\frac{\omega_{1}+\omega_{3}-\omega_{2}}{2}\right) \Gamma^{2}\left(\frac{\omega_{2}+\omega_{3}-\omega_{1}}{2}\right) \Gamma^{2}\left(\frac{\omega_{1}+\omega_{2}-\omega_{3}}{2}\right) \\
& \left.\quad \times a_{12}^{\omega_{3}-\omega_{1}-\omega_{2}} a_{13}^{\omega_{2}-\omega_{1}-\omega_{3}} a_{23}^{\omega_{1}-\omega_{2}-\omega_{3}}\right) \\
& \quad \times\left[e^{i \pi\left(\omega_{1}+\omega_{2}-\omega_{3}\right)}+e^{i \pi\left(\omega_{2}+\omega_{3}-\omega_{1}\right)}+e^{i \pi\left(\omega_{1}+\omega_{3}-\omega_{2}\right)}-e^{i \pi\left(\omega_{1}+\omega_{2}+\omega_{3}\right)}-2\right]
\end{aligned}
$$

Using these formulas with $a_{i j}^{2}=\frac{z_{i j_{t}}^{2}}{s_{i j}}$ it is easy to get

$$
\begin{aligned}
& \int_{-\infty}^{\infty} d u_{1} d v_{1}\left[\left(u_{1}-v_{1}\right)^{-\omega_{1}} \theta\left(u_{1}-v_{1}\right)+u_{1} \leftrightarrow v_{1}\right] \int_{-\infty}^{\infty} d u_{2} d v_{2}\left[\left(u_{2}-v_{2}\right)^{-\omega_{2}} \theta\left(u_{2}-v_{2}\right)\right. \\
& \left.+u_{2} \leftrightarrow v_{2}\right] \int_{-\infty}^{\infty} d u_{3} d v_{3}\left[\left(u_{3}-v_{3}\right)^{-\omega_{3}} \theta\left(u_{3}-v_{3}\right)+u_{3} \leftrightarrow v_{3}\right] \\
& \quad \times \frac{1}{\left(u_{1} v_{2} s_{12}+z_{12}^{2}+i \epsilon\right)\left(v_{1} u_{3} s_{13}+z_{13}^{2}+i \epsilon\right)\left(u_{2} v_{3} s_{23}+z_{23}^{2}+i \epsilon\right)} \\
& =\frac{1}{s_{12} s_{13} s_{23}} \Phi\left(\omega_{1}, \omega_{2}, \omega_{3}\right) \Gamma\left(1-\omega_{1}\right) \Gamma\left(1-\omega_{2}\right) \Gamma\left(1-\omega_{3}\right) \Gamma^{2}\left(\frac{\omega_{1}+\omega_{2}-\omega_{3}}{2}\right) \\
& \quad \times \Gamma^{2}\left(\frac{\omega_{2}+\omega_{3}-\omega_{1}}{2}\right) \Gamma^{2}\left(\frac{\omega_{1}+\omega_{3}-\omega_{2}}{2}\right)\left(\frac{s_{12}}{z_{12}^{2}}\right)^{\frac{\omega_{1}+\omega_{2}-\omega_{3}}{2}}\left(\frac{s_{13}}{z_{13}^{2}}\right)^{\frac{\omega_{1}+\omega_{3}-\omega_{2}}{2}}\left(\frac{s_{23}}{z_{23}^{2}}\right)^{\frac{\omega_{2}+\omega_{3}-\omega_{1}}{2}}
\end{aligned}
$$


where

$$
\begin{aligned}
\Phi & \left(\omega_{1}, \omega_{2}, \omega_{3}\right) \\
= & -4 \sin \frac{\pi \omega_{1}}{2} \sin \frac{\pi}{2}\left(\omega_{2}+\omega_{3}-\omega_{1}\right) \cos \frac{\pi}{2}\left(\omega_{2}-\omega_{3}\right)-4 \sin \frac{\pi \omega_{2}}{2} \sin \frac{\pi}{2}\left(\omega_{1}+\omega_{3}-\omega_{2}\right) \\
& \times \cos \frac{\pi}{2}\left(\omega_{1}-\omega_{3}\right)-4 \sin \frac{\pi \omega_{3}}{2} \sin \frac{\pi}{2}\left(\omega_{1}+\omega_{2}-\omega_{3}\right) \cos \frac{\pi}{2}\left(\omega_{1}-\omega_{2}\right) \\
& +e^{i \pi\left(\omega_{1}+\omega_{2}-\omega_{3}\right)}+e^{i \pi\left(\omega_{2}+\omega_{3}-\omega_{1}\right)}+e^{i \pi\left(\omega_{1}+\omega_{3}-\omega_{2}\right)}-e^{i \pi\left(\omega_{1}+\omega_{2}+\omega_{3}\right)}-2
\end{aligned}
$$

Now, differentiating eq. (B.8) two times with respect to each $x_{i j_{t}}^{2}$ one obtains

$$
\begin{aligned}
& \left\langle\mathcal{F}_{n_{1}}\left(\omega_{1}, z_{1_{t}}\right) \mathcal{F}_{\omega_{2}, n_{2}}\left(z_{2_{t}}\right) \mathcal{F}_{\omega_{3}, n_{3}}\left(z_{3_{t}}\right)\right\rangle \\
& =\frac{N_{c}^{2}-1}{32 \pi^{6} z_{12}^{2} z_{13}^{2} z_{23}^{2}} \Gamma\left(\frac{\omega_{1}+\omega_{2}-\omega_{3}}{2}\right) \Gamma\left(\frac{\omega_{2}+\omega_{3}-\omega_{1}}{2}\right) \Gamma\left(\frac{\omega_{1}+\omega_{3}-\omega_{2}}{2}\right) \\
& \times \Gamma\left(\frac{\omega_{1}+\omega_{2}-\omega_{3}}{2}+2\right) \Gamma\left(\frac{\omega_{2}+\omega_{3}-\omega_{1}}{2}+2\right) \Gamma\left(\frac{\omega_{1}+\omega_{3}-\omega_{2}}{2}+2\right) \Phi\left(\omega_{1}, \omega_{2}, \omega_{3}\right) \\
& \times \Gamma\left(1-\omega_{1}\right) \Gamma\left(1-\omega_{2}\right) \Gamma\left(1-\omega_{3}\right)\left(\frac{s_{12}}{z_{12}^{2}}\right)^{\frac{\omega_{1}+\omega_{2}-\omega_{3}}{2}}\left(\frac{s_{13}}{z_{13}^{2}}\right)^{\frac{\omega_{1}+\omega_{3}-\omega_{2}}{2}}\left(\frac{s_{23}}{z_{23}^{2}}\right)^{\frac{\omega_{2}+\omega_{3}-\omega_{1}}{2}}
\end{aligned}
$$

A quick check of this formula can be obtained by eq. (3.4) which states that as $\omega_{i} \rightarrow 1$ the coefficient in front of $\Gamma\left(1-\omega_{1}\right) \Gamma\left(1-\omega_{2}\right) \Gamma\left(1-\omega_{3}\right)$ is represented by the three-point correlator of local two-gluon operators

$$
\int d u d v d w\left\langle F_{n_{1} \xi}^{a} F_{n_{1}}^{a \xi}\left(u n_{1}+x_{1 t}\right) F_{n_{2} \eta}^{b} F_{n_{2}}^{b \eta}\left(v n_{2}+x_{2 t}\right) F_{n_{3} \zeta}^{c} F_{n_{3}}^{c \zeta}\left(w n_{3}+x_{3 t}\right)\right.
$$

Using tree-level correlator

$$
\begin{aligned}
& \left\langle F_{n_{1}}^{a \mu} F_{n_{1} \mu}^{a}\left(u_{1} n_{1}+x_{1 t}\right) F_{n_{2}}^{b \nu} F_{n_{2} \nu}^{b}\left(u_{2} n_{2}+x_{2 t}\right) F_{n_{3}}^{c \lambda} F_{n_{3} \lambda}^{c}\left(u_{3} n_{3}+x_{3 t}\right)\right\rangle^{\text {tree }} \\
& =\frac{2\left(N_{c}^{2}-1\right) s_{12} s_{13} s_{23} x_{12 t}^{2} x_{13 t}^{2} x_{23 t}^{2}}{\pi^{6}\left(u v s_{12}+x_{12 t}^{2}+i \epsilon\right)^{3}\left(u w s_{13}+x_{13 t}^{2}+i \epsilon\right)^{3}\left(v w s_{23}+x_{23 t}^{2}+i \epsilon\right)^{3}}
\end{aligned}
$$

and the integral

$$
\int d u d v d w \frac{1}{[u v+a+i \epsilon][v w+b+i \epsilon][u w+c+i \epsilon]}=-\frac{2 \pi^{3}}{\sqrt{a b c}}
$$

one obtains

$$
\begin{aligned}
& \int d u d v d w\left\langle F_{n_{1} \xi}^{a} F_{n_{1}}^{a \xi}\left(u n_{1}+x_{1 t}\right) F_{n_{2} \eta}^{b} F_{n_{2}}^{b \eta}\left(v n_{2}+x_{2 t}\right) F_{n_{3} \zeta}^{c} F_{n_{3}}^{c \zeta}\left(w n_{3}+x_{3 t}\right)\right. \\
& =-\frac{27\left(N_{c}^{2}-1\right) \sqrt{s_{12} s_{13} s_{23}}}{128 \pi^{3}\left(x_{12 t}^{2} x_{13 t}^{2} x_{23 t}^{2}\right)^{\frac{3}{2}}}
\end{aligned}
$$

which agrees with eq. (B.10) at $\omega_{i} \rightarrow 1$ since $\Phi(1,1,1)=-16$.

For the BFKL limit we need the behavior of the tree-level correlator (B.10) as $\omega_{i} \rightarrow 0$. It is easy to see that at small $\omega_{i}$

$$
\Phi\left(\omega_{1}, \omega_{2}, \omega_{3}\right)=4 i \pi^{3} \omega_{1} \omega_{2} \omega_{3}-\pi^{4} \omega_{1} \omega_{2} \omega_{3}\left(\omega_{1}+\omega_{2}+\omega_{3}\right)+O\left(\omega^{5}\right)
$$




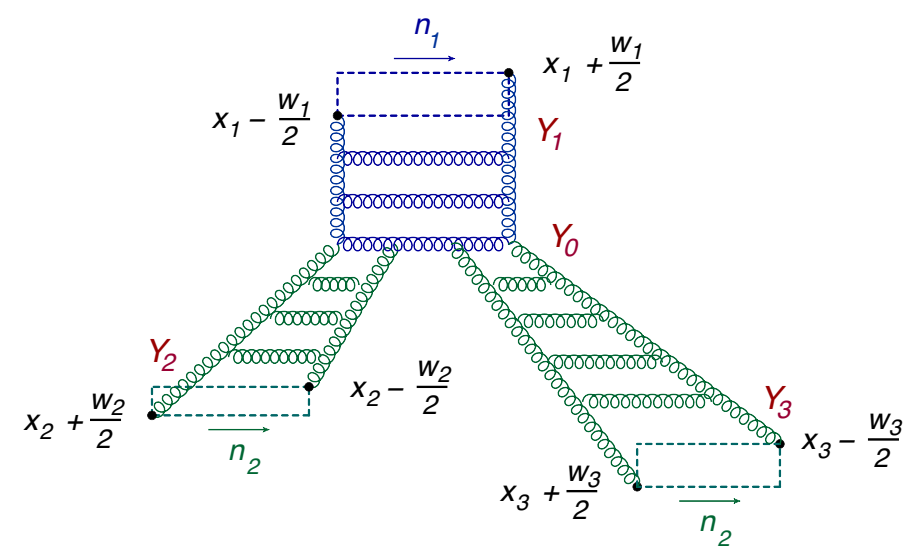

Figure 17. Triple BFKL evolution at $n_{2}=n_{3}$ with BK vertex at the rapidity $Y_{0}$.

and therefore

$$
\begin{aligned}
& \left\langle\mathcal{F}_{n_{1}}\left(\omega_{1}, z_{1 t}\right) \mathcal{F}_{\omega_{2}, n_{2}}\left(z_{2 t}\right) \mathcal{F}_{\omega_{3}, n_{3}}\left(z_{3 t}\right)\right\rangle^{\text {tree }} \\
& =\frac{i\left(N_{c}^{2}-1\right)}{\pi^{3} z_{12}^{2} z_{13}^{2} z_{23}^{2}} \frac{\omega_{1} \omega_{2} \omega_{3}\left[1+\frac{i \pi}{4}\left(\omega_{1}+\omega_{2}+\omega_{3}\right)+O\left(\omega^{2}\right)\right]}{\left(\omega_{1}+\omega_{2}-\omega_{3}\right)\left(\omega_{2}+\omega_{3}-\omega_{1}\right)\left(\omega_{1}+\omega_{3}-\omega_{2}\right)} \\
& \quad \times\left(\frac{s_{12}}{z_{12}^{2}}\right)^{\frac{\omega_{1}+\omega_{2}-\omega_{3}}{2}}\left(\frac{s_{13}}{z_{13}^{2}}\right)^{\frac{\omega_{1}+\omega_{3}-\omega_{2}}{2}}\left(\frac{s_{23}}{z_{23}^{2}}\right)^{\frac{\omega_{2}+\omega_{3}-\omega_{1}}{2}}
\end{aligned}
$$

which corresponds to

$$
\left.F\left(\omega_{i}, g^{2}\right)\right|_{g^{2}=0}=1+\frac{i \pi}{4}\left(\omega_{1}+\omega_{2}+\omega_{3}\right)+O\left(\omega^{2}\right)
$$

in the notations of eq. (3.14) parametrization.

As demonstrated in the next section, the singularities at $\omega_{i}=\omega_{j}+\omega_{k}$ originate from boost invariance of the correlator of three light-ray operators at $n_{j}=n_{k}$. Note, however, that such singularity is absent in the correlator of three local operators $F_{n}^{j}\left(x_{t}\right)$ (see eq. (2.4) for definition) since $\Phi\left(j_{i}, j_{k}, j_{i}+j_{k}\right)=0$ for integer $j$ 's.

\section{Boost invariance and singularities of structure constants}

As we mentioned above, the singularities at $\omega_{i}=\omega_{j}+\omega_{k}$ are related to boost invariance. To demonstrate this, let us follow ref. [26] and consider the correlator of Wilson frame in $n_{1}$ direction and two Wilson frames in $n_{2}$ directions, see figure 17

$$
\left\langle\mathcal{F}_{n_{1}}^{j_{1}}\left(x_{1 t}+\frac{w_{1 t}}{2}, x_{1 t}-\frac{w_{1 t}}{2}\right) \mathcal{F}_{n_{2}}^{j_{2}}\left(x_{2 t}+\frac{w_{2 t}}{2}, x_{2 t}-\frac{w_{2 t}}{2}\right) \mathcal{F}_{n_{2}}^{j_{3}}\left(x_{3 t}+\frac{w_{3 t}}{2}, x_{3 t}-\frac{w_{3 t}}{2}\right)\right\rangle
$$

As we discussed in section 5.2, this correlator in the Regge limit can be represented by the correlator of three conformal dipoles, one in $n_{1}$ direction and two in $n_{2}$ directions. We get 
from eq. (5.26)

$$
\begin{aligned}
& \left\langle\mathcal{F}_{n_{1}}^{j_{1}}\left(x_{1 t}+\frac{w_{1 t}}{2}, x_{1 t}-\frac{w_{1 t}}{2}\right) \mathcal{F}_{n_{2}}^{j_{2}}\left(x_{2 t}+\frac{w_{2 t}}{2}, x_{2 t}-\frac{w_{2 t}}{2}\right) \mathcal{F}_{n_{2}}^{j_{3}}\left(x_{3 t}+\frac{w_{3 t}}{2}, x_{3 t}-\frac{w_{3 t}}{2}\right)\right\rangle \\
& =-i \frac{N_{c}^{6}}{\pi^{9}} \int_{0}^{\infty} \frac{d l_{1} d l_{2} d l_{3}}{l_{1} l_{2} l_{3}} l_{1}^{-\omega_{1}} l_{2}^{-\omega_{2}} l_{3}^{-\omega_{3}} \int d \nu_{1} d \nu_{2} d \nu_{3}\left(w_{1 t}^{2}\right)^{-\frac{1}{2}+i \nu_{1}}\left(w_{2 t}^{2}\right)^{-\frac{1}{2}+i \nu_{2}}\left(w_{3 t}^{2}\right)^{-\frac{1}{2}+i \nu_{3}} \\
& \quad \times\left\langle\mathcal{U}_{\mathrm{conf}}^{Y_{1}}\left(x_{1 t},-\nu_{1}\right) \mathcal{V}_{\mathrm{conf}}^{Y_{2}}\left(x_{2 t},-\nu_{2}\right) \mathcal{V}_{\mathrm{conf}}^{Y_{3}}\left(x_{3 t},-\nu_{3}\right)\right\rangle \prod_{i=1,2,3} \nu_{i} \frac{2^{-4 i \nu_{i}} \Gamma\left(\frac{3}{2}+i \nu_{i}\right) \Gamma\left(1-i \nu_{i}\right)}{\Gamma\left(\frac{3}{2}-i \nu_{i}\right) \Gamma\left(1+i \nu_{i}\right)}
\end{aligned}
$$

where $Y_{i} \simeq \ln l_{i}+\frac{1}{2} \ln s m_{\perp}^{2}\left(s \equiv s_{12}\right)$.

As discussed in refs. [26] and [27], the BK equation for color dipoles leads to the following structure of the correlator of a conformal dipole in $n_{1}$ direction and two dipoles in $n_{2}$ direction:

$$
\int d Y_{0} \theta\left(Y_{0}+Y_{2}\right) \theta\left(Y_{0}+Y_{3}\right)\left(\text { evolution } \mathcal{U}^{Y_{1}} \rightarrow \mathcal{U}^{Y_{0}}\right) \otimes\left(\text { BK vertex at } Y_{0}\right) \otimes\left(\begin{array}{l}
\left\langle\mathcal{U}^{Y_{0}} \mathcal{V}^{Y_{2}}\right\rangle \\
\left\langle\mathcal{U}^{Y_{0}} \mathcal{V}^{Y_{3}}\right\rangle
\end{array}\right)
$$

where the integral over $Y_{0}$ comes from the fact that the splitting of one dipole into two (described by the BK vertex) can occur at any rapidity between the $n_{1}$ dipole and the most energetic of $n_{2}$ dipoles. Specifically, $\theta\left(Y_{0}+Y_{i}\right)$ reflects the fact that there should be sufficient energy between dipoles $\mathcal{U}^{Y_{0}}$ and $\mathcal{V}^{Y_{i}}$ to apply high-energy approximation, see the footnote 9 at page 15 .

Rewrtiting eq. (18) from ref. [26] in terms of conformal dipoles, one gets

$$
\begin{aligned}
& \left\langle\mathcal{U}_{\mathrm{conf}}^{Y_{1}}\left(x_{1 t},-\nu_{1}\right) \mathcal{V}_{\mathrm{conf}}^{Y_{2}}\left(x_{2 t},-\nu_{2}\right) \mathcal{V}_{\mathrm{conf}}^{Y_{3}}\left(x_{3 t},-\nu_{3}\right)\right\rangle=-\frac{\alpha_{s}^{5} N_{c}}{4 \pi^{4}} \frac{1}{\left(\frac{1}{4}+\nu_{2}^{2}\right)^{2}\left(\frac{1}{4}+\nu_{3}^{2}\right)^{2}} \\
& \quad \times \int_{-\infty}^{Y_{1}} d Y_{0} \int d Y_{2} d Y_{3} \theta\left(Y_{0}+Y_{2}\right) \theta\left(Y_{0}+Y_{3}\right) e^{\aleph\left(\nu_{1}\right)\left(Y_{1}-Y_{0}\right)+\aleph\left(\nu_{2}\right)\left(Y_{0}+Y_{2}\right)+\aleph\left(\nu_{3}\right)\left(Y_{0}+Y_{3}\right)} \\
& \quad \times \int \frac{d^{2} x_{4} d^{2} x_{5} d^{2} x_{6}}{x_{45}^{2} x_{56}^{2} x_{46}^{2}}\left(\frac{x_{45}^{2}}{x_{14}^{2} x_{15}^{2}}\right)^{\frac{1}{2}+i \nu_{1}}\left(\frac{x_{56}^{2}}{x_{25}^{2} x_{36}^{2}}\right)^{\frac{1}{2}+i \nu_{2}}\left(\frac{x_{46}^{2}}{x_{34}^{2} x_{36}^{2}}\right)^{\frac{1}{2}+i \nu_{3}}+O\left(\frac{1}{N_{c}}\right)
\end{aligned}
$$

where two-dimensional integrals go over transverse directions orthogonal to both $n_{1}$ and $n_{2}$. Combining eqs. (C.2), (C.3), and (C.4) one obtains

$$
\begin{aligned}
& \left\langle\mathcal{F}_{n_{1}}^{j_{1}}\left(x_{1 t}+\frac{w_{1 t}}{2}, x_{1 t}-\frac{w_{1 t}}{2}\right) \mathcal{F}_{n_{2}}^{j_{2}}\left(x_{2 t}+\frac{w_{2 t}}{2}, x_{2 t}-\frac{w_{2 t}}{2}\right) \mathcal{F}_{n_{2}}^{j_{3}}\left(x_{3 t}+\frac{w_{3 t}}{2}, x_{3 t}-\frac{w_{3 t}}{2}\right)\right\rangle \\
& =i \frac{\alpha_{s}^{5} N_{c}^{7}}{4 \pi^{13}} \int \frac{d \nu_{1} d \nu_{2} d \nu_{3}}{\left(\frac{1}{4}+\nu_{2}^{2}\right)^{2}\left(\frac{1}{4}+\nu_{3}^{2}\right)^{2}} \frac{2 \pi \delta\left(\omega_{1}-\omega_{2}-\omega_{3}\right)\left(\frac{s}{m_{\perp}^{2}}\right)^{\frac{\omega_{1}+\omega_{2}+\omega_{3}}{2}}}{\left[\omega_{1}-\aleph\left(\nu_{1}\right)\right]\left[\omega_{2}-\aleph\left(\nu_{2}\right)\right]\left[\omega_{3}-\aleph\left(\nu_{3}\right)\right]} \\
& \quad \times\left(w_{1 t}^{2}\right)^{-\frac{1}{2}+i \nu_{1}}\left(w_{2 t}^{2}\right)^{-\frac{1}{2}+i \nu_{2}}\left(w_{3 t}^{2}\right)^{-\frac{1}{2}+i \nu_{3}} \prod_{k=1,2,3} \nu_{k} \frac{2^{-4 i \nu_{k}} \Gamma\left(\frac{3}{2}+i \nu_{k}\right) \Gamma\left(1-i \nu_{k}\right)}{\Gamma\left(\frac{3}{2}-i \nu_{k}\right) \Gamma\left(1+i \nu_{k}\right)} \\
& \quad \times \int \frac{d^{2} x_{4} d^{2} x_{5} d^{2} x_{6}}{x_{45}^{2} x_{56}^{2} x_{46}^{2}}\left(\frac{x_{45}^{2}}{x_{14}^{2} x_{15}^{2}}\right)^{\frac{1}{2}+i \nu_{1}}\left(\frac{x_{56}^{2}}{x_{25}^{2} x_{36}^{2}}\right)^{\frac{1}{2}+i \nu_{2}}\left(\frac{x_{46}^{2}}{x_{34}^{2} x_{36}^{2}}\right)^{\frac{1}{2}+i \nu_{3}}
\end{aligned}
$$


Here $2 \pi \delta\left(\omega_{1}-\omega_{2}-\omega_{3}\right)$ comes from the longitudinal integral

$$
\begin{aligned}
& \int_{0}^{\infty} \frac{d l_{1} d l_{2} d l_{3}}{l_{1} l_{2} l_{3}} l_{1}^{-\omega_{1}} l_{2}^{-\omega_{2}} l_{3}^{-\omega_{3}} \int_{-\infty}^{Y_{1}} d Y_{0} \int d Y_{2} d Y_{3} \theta\left(Y_{0}+Y_{2}\right) \theta\left(Y_{0}+Y_{3}\right) \\
& \quad \times e^{\aleph\left(\nu_{1}\right)\left(Y_{1}-Y_{0}\right)+\aleph\left(\nu_{2}\right)\left(Y_{0}+Y_{2}\right)+\aleph\left(\nu_{3}\right)\left(Y_{0}+Y_{3}\right)} \\
& =\frac{2 \pi \delta\left(\omega_{1}-\omega_{2}-\omega_{3}\right)\left(\frac{s}{m_{\perp}^{2}}\right)^{\frac{\omega_{1}+\omega_{2}+\omega_{3}}{2}}}{\left[\omega_{1}-\aleph\left(\nu_{1}\right)\right]\left[\omega_{2}-\aleph\left(\nu_{2}\right)\right]\left[\omega_{3}-\aleph\left(\nu_{3}\right)\right]} \int d Y_{0} e^{-i\left(\omega_{1}-\omega_{2}-\omega_{3}\right) Y_{0}} \\
& \rightarrow \frac{2 \pi \delta\left(\omega_{1}-\omega_{2}-\omega_{3}\right)\left(\frac{s}{m_{\perp}^{2}}\right)^{\frac{\omega_{1}+\omega_{2}+\omega_{3}}{2}}}{\left[\omega_{1}-\aleph\left(\nu_{1}\right)\right]\left[\omega_{2}-\aleph\left(\nu_{2}\right)\right]\left[\omega_{3}-\aleph\left(\nu_{3}\right)\right]}
\end{aligned}
$$

Strictly speaking, the integral over $Y_{0}$ is divergent so we need some regularization to understand it. Following ref. [26] we take $n_{2} \neq n_{3}$ but $n_{1} \cdot n_{2} \simeq n_{1} \cdot n_{3}$. We can use our formulas for $n_{2}=n_{3}$ case until longitudinal distances between frames " 2 " and " 3 " are smaller than typical transverse separation $x_{i j \perp}^{2} \sim m_{\perp}^{-2}$, i.e. when $l_{2} l_{3} s_{23} \leq m_{\perp}^{-2}$. In terms of rapidities $Y_{2}$ and $Y_{3}$ this restriction means $Y_{2}+Y_{3} \leq \ln \frac{s_{12}}{s_{23}}$ so instead of eq. (C.6) we get

$$
\begin{aligned}
& \int_{-\infty}^{Y_{1}} d Y_{0} \int d Y_{2} d Y_{3} \theta\left(Y_{0}+Y_{2}\right) \theta\left(Y_{0}+Y_{3}\right) \theta\left(\ln \frac{s_{12}}{s_{23}}-Y_{2}-Y_{3}\right) \\
& \times e^{\aleph\left(\nu_{1}\right)\left(Y_{1}-Y_{0}\right)+\aleph\left(\nu_{2}\right)\left(Y_{0}+Y_{2}\right)+\aleph\left(\nu_{3}\right)\left(Y_{0}+Y_{3}\right)} \\
& =\frac{\left(\frac{s_{23}}{s_{12}}\right)^{\frac{\omega_{2}+\omega_{3}-\omega_{1}}{2}}}{\left(\omega_{1}-\omega_{2}-\omega_{3}\right)\left(\omega_{1}-\aleph_{1}\right)\left(\omega_{2}-\aleph_{2}+\frac{\omega_{1}-\omega_{2}-\omega_{3}}{2}\right)\left(\omega_{3}-\aleph_{3}+\frac{\omega_{1}-\omega_{2}-\omega_{3}}{2}\right)} \\
& \omega_{2}+\underline{\omega}_{3} \rightarrow \omega_{1} \frac{\left(\frac{s_{23}}{s_{12}}\right)^{\frac{\omega_{2}+\omega_{3}-\omega_{1}}{2}}}{\left(\omega_{1}-\omega_{2}-\omega_{3}\right)\left(\omega_{1}-\aleph_{1}\right)\left(\omega_{2}-\aleph_{2}\right)\left(\omega_{3}-\aleph_{3}\right)} \text {. }
\end{aligned}
$$

Thus,

$$
2 \pi \delta\left(\omega_{1}-\omega_{2}-\omega_{3}\right) \Leftrightarrow \lim _{n_{3} \rightarrow n_{2}} \frac{\left(\frac{n_{2} \cdot n_{3}}{n_{1} \cdot n_{2}}\right)^{\frac{\omega_{2}+\omega_{3}-\omega_{1}}{2}}}{\left(\omega_{1}-\omega_{2}-\omega_{3}\right)}
$$

Let us emphasize that the divergence over $Y_{0}$ in r.h.s. of eq. (C.7) leading to this $\delta$-function comes from boost invariance: at $n_{2}=n_{3}$ one can multiply $n_{1}$ by some $\lambda$ and $n_{2}$ by $\lambda^{-1}$ and the correlator (C.2) will not change. Thus, the singularity at $\omega_{1}=\omega_{2}+\omega_{3}$ is of general nature and should be present in a general formula (3.14). Note, however, that for the correlator of 3 local "forward" operators these singularities seem to disappear, see the discussion in the end of section B.

To compare with the result (6.44) for $n_{2} \neq n_{3}$ let us finish the calculation in this $n_{2}=n_{3}$ case. The transverse integral was calculated in ref. [31] and the result is

$$
\begin{aligned}
& \int \frac{d^{2} x_{4} d^{2} x_{5} d^{2} x_{6}}{x_{45}^{2} x_{56}^{2} x_{46}^{2}}\left(\frac{x_{45}^{2}}{x_{14}^{2} x_{15}^{2}}\right)^{\frac{1}{2}+i \nu_{1}}\left(\frac{x_{56}^{2}}{x_{25}^{2} x_{36}^{2}}\right)^{\frac{1}{2}+i \nu_{2}}\left(\frac{x_{46}^{2}}{x_{34}^{2} x_{36}^{2}}\right)^{\frac{1}{2}+i \nu_{3}} \\
& =\frac{\pi^{3} \Omega\left(\frac{1}{2}+i \nu_{1}, \frac{1}{2}+i \nu_{2}, \frac{1}{2}+i \nu_{3}\right)}{\left(x_{12}^{2}\right)^{\frac{1}{2}+i \nu_{1}+i \nu_{2}-i \nu_{3}}\left(x_{13}^{2}\right)^{\frac{1}{2}+i \nu_{1}+i \nu_{3}-i \nu_{2}}\left(x_{23}^{2}\right)^{\frac{1}{2}+i \nu_{2}+i \nu_{3}-i \nu_{1}}}
\end{aligned}
$$


where $\Omega$ is related to Meyer G-function, see the explicit expression in ref. [31] (for convenience, we extracted factor $\pi^{3}$ from the definition in ref. [31]).

Rewriting the intgral (C.9) in terms of $\gamma_{i}=2 i \nu_{i}-1$ one obtains

$$
\begin{aligned}
\left\langle\mathcal{F}_{n_{1}}^{j_{1}}(\right. & \left.\left.x_{1 t}+\frac{w_{1 t}}{2}, x_{1 t}-\frac{w_{1 t}}{2}\right) \mathcal{F}_{n_{2}}^{j_{2}}\left(x_{2 t}+\frac{w_{2 t}}{2}, x_{2 t}-\frac{w_{2 t}}{2}\right) \mathcal{F}_{n_{2}}^{j_{3}}\left(x_{3 t}+\frac{w_{3 t}}{2}, x_{3 t}-\frac{w_{3 t}}{2}\right)\right\rangle \\
= & -i \frac{\alpha_{s}^{5} N_{c}^{7}}{64 \pi^{10}} \frac{2 \pi \delta\left(\omega_{1}-\omega_{2}-\omega_{3}\right)}{x_{12 t}^{2} x_{13 t}^{2} x_{23 t}^{2}} \int_{-1-i \infty}^{-1+i \infty} \frac{d \gamma_{1} d \gamma_{2} d \gamma_{3}}{\gamma_{2}^{2} \gamma_{3}^{2}\left(2+\gamma_{2}\right)^{2}\left(2+\gamma_{3}\right)^{2}} \\
& \times \prod_{k=1}^{3} \frac{\left(1+\gamma_{k}\right) 2^{-2 \gamma_{k}} \Gamma\left(2+\frac{\gamma_{k}}{2}\right) \Gamma\left(\frac{1}{2}-\frac{\gamma_{k}}{2}\right)}{\Gamma\left(1-\frac{\gamma_{k}}{2}\right) \Gamma\left(\frac{3}{2}+\frac{\gamma_{k}}{2}\right)} \frac{\Omega\left(1-\frac{\gamma_{1}}{2}, 1-\frac{\gamma_{2}}{2}, 1-\frac{\gamma_{3}}{2}\right)}{\left[\omega_{1}-\tilde{\aleph}\left(\gamma_{1}\right)\right]\left[\omega_{2}-\tilde{\aleph}\left(\gamma_{2}\right)\right]\left[\omega_{3}-\tilde{\aleph}\left(\gamma_{3}\right)\right]} \\
& \times\left(\frac{w_{1 t}^{2} x_{23 t}^{2}}{x_{12 t}^{2} x_{13 t}^{2}}\right)^{\frac{\gamma_{1}}{2}}\left(\frac{w_{2 t}^{2} x_{13 t}^{2}}{x_{12 t}^{2} x_{23 t}^{2}}\right)^{\frac{\gamma_{2}}{2}}\left(\frac{w_{3 t}^{2} x_{12 t}^{2}}{x_{13 t}^{2} x_{23 t}^{2}}\right)^{\frac{\gamma_{3}}{2}}\left(\frac{s}{m_{\perp}^{2}}\right)^{\frac{\omega_{1}+\omega_{2}+\omega_{3}}{2}}
\end{aligned}
$$

where $\gamma_{i}=2 i \nu_{i}-1$ similarly to eq. (6.39).

Taking residues at $\gamma_{i}^{*}$ (roots of the equation (6.43)) one obtains ${ }^{17}$

$$
\begin{aligned}
\left\langle\mathcal{F}_{n_{1}}^{j_{1}}\left(x_{1 t}+\frac{w_{1 t}}{2}, x_{1 t}-\frac{w_{1 t}}{2}\right) \mathcal{F}_{n_{2}}^{j_{2}}\left(x_{2 t}+\frac{w_{2 t}}{2}, x_{2 t}-\frac{w_{2 t}}{2}\right) \mathcal{F}_{n_{2}}^{j_{3}}\left(x_{3 t}+\frac{w_{3 t}}{2}, x_{3 t}-\frac{w_{3 t}}{2}\right)\right\rangle \\
=8 g^{10} \frac{N_{c}^{2}}{\pi^{2}} 2 \pi \delta\left(\omega_{1}-\omega_{2}-\omega_{3}\right)\left(\frac{s}{x_{12 t}^{2}}\right)^{\frac{\omega_{1}+\omega_{2}-\omega_{3}}{2}}\left(\frac{s}{x_{13 t}^{2}}\right)^{\frac{\omega_{1}+\omega_{3}-\omega_{2}}{2}}\left(\frac{s}{x_{23 t}^{2}}\right)^{\frac{\omega_{2}+\omega_{3}-\omega_{1}}{2}} \\
\quad \times \Omega\left(1-\frac{\gamma_{1}^{*}}{2}, 1-\frac{\gamma_{2}^{*}}{2}, 1-\frac{\gamma_{3}^{*}}{2}\right) \frac{1}{x_{12 t}^{2} x_{13 t}^{2} x_{23 t}^{2}}\left(\frac{w_{1 t}^{2} x_{23 t}^{2}}{x_{12 t}^{2} x_{13 t}^{2}}\right)^{\frac{\gamma_{1}^{*}}{2}}\left(\frac{w_{2 t}^{2} x_{13 t}^{2}}{x_{12 t}^{2} x_{23 t}^{2}}\right)^{\frac{\gamma_{2}^{*}}{2}}\left(\frac{w_{3 t}^{2} x_{12 t}^{2}}{x_{13 t}^{2} x_{23 t}^{2}}\right)^{\frac{\gamma_{3}^{*}}{2}} \\
\quad \times \frac{1}{\gamma_{2}^{* 2} \gamma_{3}^{* 2}\left(1+\frac{\gamma_{2}^{*}}{2}\right)^{2}\left(1+\frac{\gamma_{3}^{*}}{2}\right)^{2}} \prod_{k=1}^{3} \frac{\left(1+\gamma_{k}^{*}\right) 2^{-2 \gamma_{k}^{*}} \Gamma\left(2+\frac{\gamma_{k}^{*}}{2}\right) \Gamma\left(\frac{1}{2}-\frac{\gamma_{k}^{*}}{2}\right)}{\Gamma\left(1-\frac{\gamma_{k}^{*}}{2}\right) \Gamma\left(\frac{3}{2}+\frac{\gamma_{k}^{*}}{2}\right) \aleph^{\prime}\left(\gamma_{k}^{*}\right)}
\end{aligned}
$$

Here again we replaced $\left(\frac{s}{m_{\perp}^{2}}\right)^{\frac{\omega_{1}+\omega_{2}+\omega_{3}}{2}}$ by $\left(\frac{s}{x_{12 t}^{2}}\right)^{\frac{\omega_{1}+\omega_{2}-\omega_{3}}{2}}\left(\frac{s}{x_{13 t}^{2}}\right)^{\frac{\omega_{1}+\omega_{3}-\omega_{2}}{2}}\left(\frac{s}{x_{23 t}^{2}}\right)^{\frac{\omega_{2}+\omega_{3}-\omega_{1}}{2}}$ which is within LLA accuracy. This result agrees with eq. (30) from ref. [26]. In terms of structure constant (3.14) we have

$$
\begin{aligned}
& F\left[\gamma^{*}\left(\omega_{1}, g^{2}\right), \gamma^{*}\left(\omega_{2}, g^{2}\right), \gamma^{*}\left(\omega_{3}, g^{2}\right)\right] \\
& \stackrel{\omega_{1}=\omega_{2}+\omega_{3}}{=} 8 i g^{10} \frac{N_{c}^{2}}{\pi^{2}} \frac{\Omega\left(1-\frac{\gamma_{1}^{*}}{2}, 1-\frac{\gamma_{2}^{*}}{2}, 1-\frac{\gamma_{3}^{*}}{2}\right)}{\gamma_{2}^{* 2} \gamma_{3}^{* 2}\left(1+\frac{\gamma_{2}^{*}}{2}\right)^{2}\left(1+\frac{\gamma_{3}^{*}}{2}\right)^{2}} \prod_{k=1}^{3} \frac{\left(1+\gamma_{k}^{*}\right) 2^{-2 \gamma_{k}^{*}} \Gamma\left(2+\frac{\gamma_{k}^{*}}{2}\right) \Gamma\left(\frac{1}{2}-\frac{\gamma_{k}^{*}}{2}\right)}{\Gamma\left(1-\frac{\gamma_{k}^{*}}{2}\right) \Gamma\left(\frac{3}{2}+\frac{\gamma_{k}^{*}}{2}\right) \aleph^{\prime}\left(\gamma_{k}^{*}\right)}
\end{aligned}
$$

It is instructive to compare with the result (6.44) at small $\gamma_{i}^{*} \simeq-\frac{8 g^{2}}{\omega_{i}} \ll 1$. The estimate of the function $\Omega$ at small $\gamma_{i}$ reads $[26,27]$

$$
\Omega\left(1-\frac{\gamma_{1}}{2}, 1-\frac{\gamma_{2}}{2}, 1-\frac{\gamma_{3}}{2}\right)=\frac{16}{\gamma_{1} \gamma_{2} \gamma_{3}}\left(1+\frac{\gamma_{1}+\gamma_{2}}{\gamma_{3}}+\frac{\gamma_{1}+\gamma_{3}}{\gamma_{2}}+\frac{\gamma_{2}+\gamma_{3}}{\gamma_{1}}\right)+O\left(\gamma_{i}\right)
$$

\footnotetext{
${ }^{17}$ Similarly to the case of integral (6.39), the poles at $\gamma_{i}=0$ should cancel with contributions of low-order diagrams without gluon ladder(s) in figure 17.
} 
so we get

$$
\begin{aligned}
& \left\langle\mathcal{F}_{n_{1}}^{j_{1}}\left(x_{1 t}+\frac{w_{1 t}}{2}, x_{1 t}-\frac{w_{1 t}}{2}\right) \mathcal{F}_{n_{2}}^{j_{2}}\left(x_{2 t}+\frac{w_{2 t}}{2}, x_{2 t}-\frac{w_{2 t}}{2}\right) \mathcal{F}_{n_{2}}^{j_{3}}\left(x_{3 t}+\frac{w_{3 t}}{2}, x_{3 t}-\frac{w_{3 t}}{2}\right)\right\rangle \quad(\mathrm{C} .14) \\
& =-\frac{g^{2} N_{c}^{2}}{2 \pi^{2}} \omega_{1}\left[2 \pi \delta\left(\omega_{1}-\omega_{2}-\omega_{3}\right)\right]\left(\frac{w_{1 t}^{2} x_{23 t}^{2}}{x_{12 t}^{2} x_{13 t}^{2}}\right)^{\frac{\gamma_{1}^{*}}{2}}\left(\frac{w_{2 t}^{2} x_{13 t}^{2}}{x_{12 t}^{2} x_{23 t}^{2}}\right)^{\frac{\gamma_{2}^{*}}{2}}\left(\frac{w_{3 t}^{2} x_{12 t}^{2}}{x_{13 t}^{2} x_{23 t}^{2}}\right)^{\frac{\gamma_{3}^{*}}{2}}\left[1+O\left(\frac{g^{2}}{\omega}\right)\right]
\end{aligned}
$$

which corresponds to structure constant (3.14) with

$$
F=-2 i \pi g^{2}
$$

at $\omega_{1}=\omega_{2}+\omega_{3}$. This contribution to structure constant is imaginary in accordance with the fact that the physical amplitude in figure 17 is purely imaginary if left and right sides are symmetric. (The corresponding cross section describes diffractive scattering, see refs. [41, 48]). Since the leading-order structure constant $(6.47)$ is real, it is natural to assume that eq. (C.12) gives the leading contribution to the imaginary part of structure constant at $\omega_{1}=\omega_{2}+\omega_{3}$ in the BFKL limit.

\section{Calculation of $\Lambda\left(\nu_{1}, \nu_{2}, \nu_{3}\right)$}

The function $\Lambda\left(\nu_{1}, \nu_{2}, \nu_{3}\right)$ is represented by the integral (6.35). It is convenient to take $x=(1,0)$ and rewrite the integral as

$$
\begin{aligned}
\Lambda\left(\nu_{1}, \nu_{2}, \nu_{3}\right) \equiv \bar{\Lambda}\left(\epsilon_{1}, \epsilon_{2}, \epsilon_{3}\right)= & \frac{1}{\pi^{3}} \int d^{2} z_{1} \frac{d^{2} z_{2}}{\left(1-z_{2}\right)^{4}} \frac{d^{2} z_{0}}{z_{0}^{4}} \\
& \times\left[z_{12 x}^{2}+\left(z_{1 y}+z_{2 y}\right)^{2}\right]^{-\epsilon_{1}}\left[\frac{z_{20}^{2}}{\left(1-z_{2}\right)^{2}\left(1-z_{0}\right)^{2}}\right]^{-\epsilon_{2}}\left[\frac{z_{10}^{2}}{z_{1}^{2} z_{0}^{2}}\right]^{-\epsilon_{3}}
\end{aligned}
$$

where we denote $\epsilon_{i} \equiv \frac{1}{2}-i \nu_{i}$ in a view of a later estimate at $\epsilon_{i} \rightarrow 0$. Unfortunately, the integral (D.1) diverges as $\epsilon_{i} \rightarrow 0$ so we need to define it as an analytic continuation of a convergent integral

$$
\begin{aligned}
\bar{\Lambda}^{a}\left(\epsilon_{1}, \epsilon_{2}, \epsilon_{3}\right) \equiv & \frac{1}{\pi^{3}} \int d^{2} z_{0} d^{2} z_{1} d^{2} z_{2} \frac{1}{\left(\left|1-z_{0}\right|^{2}\right)^{a-\epsilon_{2}}\left(\left|1-z_{2}\right|^{2}\right)^{2-a-\epsilon_{2}}} \\
& \times\left(\bar{z}_{2}-z_{1}\right)^{-\epsilon_{1}}\left(z_{2}-\bar{z}_{1}\right)^{-\epsilon_{1}} \frac{1}{\left|z_{20}^{2}\right| \epsilon_{2}\left|z_{1}^{2}\right|^{a-\epsilon_{3}}\left|z_{10}^{2}\right| \epsilon_{3}\left|z_{0}^{2}\right|^{2-a-\epsilon_{3}}}
\end{aligned}
$$

This integral is obviously convergent if $\epsilon_{i}>0$ and $|1-a|<\epsilon_{i}$. (We will relax the condition $\epsilon_{i}>0$ later). The "Feynman diagram" integral is depicted in figure 18 the denominators being conventional 2-dim propagators (albeit with non-integer powers) except $\left[z_{12 x}^{2}+\left(z_{1 y}+\right.\right.$ $\left.\left.z_{2 y}\right)^{2}\right]^{-\epsilon_{1}}$ denoted by a dotted line.

To calculate this integral, we will rewrite $\left[z_{12 x}^{2}+\left(z_{1 y}+z_{2 y}\right)^{2}\right]$ in the denominator as $\left(\bar{z}_{2}-z_{1}\right)\left(z_{2}-\bar{z}_{1}\right)$ where $z \equiv z_{x}+i z_{y}, \bar{z} \equiv z_{x}-i z_{y}$ and use the expansion

$$
\frac{1}{\left(\bar{z}_{2}-z_{1}\right)^{\epsilon_{1}}\left(z_{2}-\bar{z}_{1}\right)^{\epsilon_{1}}}=\sum_{m, n=0}^{\infty} \frac{\Gamma\left(\epsilon_{1}+m+n\right)}{\Gamma\left(\epsilon_{1}\right) m ! n !}\left(1-\bar{z}_{2}\right)^{m} z_{1}^{n} \sum_{k, l=0}^{\infty} \frac{\Gamma\left(\epsilon_{1}+k+l\right)}{\Gamma(a) k ! l !}\left(1-z_{2}\right)^{k} \bar{z}_{1}^{l}
$$




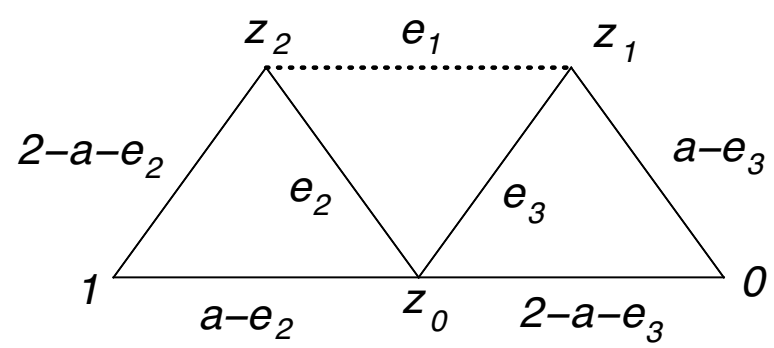

Figure 18. Correlator defining the function $\Lambda\left(\epsilon_{i}\right)\left(\equiv \Lambda\left(e_{i}\right)\right)$ in eq. (D.1). Dotted line depicts the unusial propagator $\left[z_{12 x}^{2}+\left(z_{1 y}+z_{2 y}\right)^{2}\right]^{-e_{1}}$ between points $z_{1}$ and $z_{2}$.

Next, we use the expansion (D.3), calculate the integrals, reassemble the sum over $m, n, k, l$ and continue analytically to $a=0$ in the final result.

Using the integral

$$
\int \frac{d^{2} z_{1}}{\pi} \frac{z_{1}^{m} \bar{z}_{1}^{n}}{\left|z_{2}-z_{1}\right|^{2 \alpha}\left|z_{1}\right|^{2 \beta}}=\frac{\Gamma(\alpha+\beta-n-1)}{\Gamma(\alpha) \Gamma(\beta-n)\left|z_{2}^{2}\right|^{\alpha+\beta-n-1}} z_{2}^{m-n} \frac{\Gamma(1-\alpha) \Gamma(1-\beta+m)}{\Gamma(2-\alpha-\beta+m)}
$$

one obtains after some algebra

$$
\begin{aligned}
\bar{\Lambda}^{a}\left(\epsilon_{1}, \epsilon_{2}, \epsilon_{3}\right) \\
=\frac{\sin \pi\left(a+\epsilon_{2}\right) \sin \pi \epsilon_{2} \sin \pi\left(\epsilon_{3}-a\right) \sin \pi \epsilon_{3}}{\pi^{3} \sin \pi\left(\epsilon_{2}+\epsilon_{3}\right)} \sum_{m, n, k, l=0}^{\infty} \frac{\Gamma\left(\epsilon_{1}+m+n\right)}{\Gamma\left(\epsilon_{1}\right) m ! n !} \frac{\Gamma\left(\epsilon_{1}+k+l\right)}{\Gamma\left(\epsilon_{1}\right) k ! l !} \\
\quad \times(-1)^{m+l} \frac{\Gamma\left(a+\epsilon_{2}-1+m\right) \Gamma(1-a-m)}{\Gamma\left(\epsilon_{2}\right)} \frac{\Gamma\left(1-\epsilon_{2}\right) \Gamma\left(\epsilon_{2}+a-1+k\right)}{\Gamma(a+k)} \\
\quad \times \frac{\Gamma\left(\epsilon_{3}+l+1-a\right) \Gamma(a-l-1)}{\Gamma\left(\epsilon_{3}\right)} \frac{\Gamma\left(1-\epsilon_{3}\right) \Gamma\left(1+\epsilon_{3}-a+n\right)}{\Gamma(2-a+n)} \\
\quad \times \frac{\Gamma\left(\epsilon_{2}+m\right) \Gamma\left(\epsilon_{3}+l\right)}{\Gamma\left(\epsilon_{2}+\epsilon_{3}+m+l\right)} \frac{\Gamma\left(\epsilon_{2}+k\right) \Gamma\left(\epsilon_{3}+n\right)}{\Gamma\left(\epsilon_{2}+\epsilon_{3}+k+n\right)} \\
=-\frac{\sin \pi\left(a+\epsilon_{2}\right) \sin \pi \epsilon_{2} \sin \pi\left(\epsilon_{3}-a\right) \sin \pi \epsilon_{3}}{\pi^{3} \sin \pi\left(\epsilon_{2}+\epsilon_{3}\right)} \sum_{m, n=0}^{\infty} \frac{\Gamma\left(\epsilon_{1}+m+n\right)}{\Gamma\left(\epsilon_{1}\right) m ! n !} \sum_{k, l=0}^{\infty} \frac{\Gamma\left(\epsilon_{1}+k+l\right)}{\Gamma\left(\epsilon_{1}\right) k ! l !} \\
\quad \times(-1)^{m+l} \int_{0}^{1} d u_{1} d v_{1} d u_{2} d v_{2} d t_{1} d t_{2} u_{1}^{a+\epsilon_{2}-2+m}\left(1-u_{1}\right)^{-a-m}\left(1-v_{1}\right)^{-\epsilon_{2}} v_{1}^{\epsilon_{2}+a-2+k} u_{2}^{\epsilon_{3}-a+l} \\
\quad \times\left(1-u_{2}\right)^{a-2-l}\left(1-v_{2}\right)^{-\epsilon_{3}} v_{2}^{\epsilon_{3}-a+n} t_{1}^{\epsilon_{2}-1+m}\left(1-t_{1}\right)^{\epsilon_{3}-1+l} t_{2}^{\epsilon_{2}-1+k}\left(1-t_{2}\right)^{\epsilon_{3}-1+n} \quad \text { (D.5) }
\end{aligned}
$$

Now one can reassemble the sum (D.3) and get

$$
\begin{aligned}
\bar{\Lambda}^{a}\left(\epsilon_{1}, \epsilon_{2}, \epsilon_{3}\right)= & -\frac{\sin \pi\left(a+\epsilon_{2}\right) \sin \pi \epsilon_{2} \sin \pi\left(\epsilon_{3}-a\right) \sin \pi \epsilon_{3}}{\pi^{3} \sin \pi\left(\epsilon_{2}+\epsilon_{3}\right)} \int_{0}^{1} d u_{1} d v_{1} d u_{2} d v_{2} d t_{1} d t_{2} \\
& \times u_{1}^{a+\epsilon_{2}-2}\left(1-u_{1}\right)^{\epsilon_{1}-a}\left(1-v_{1}\right)^{-\epsilon_{2}} v_{1}^{\epsilon_{2}+a-2} u_{2}^{\epsilon_{3}-a}\left(1-u_{2}\right)^{\epsilon_{1}+a-2}\left(1-v_{2}\right)^{-\epsilon_{3}} v_{2}^{\epsilon_{3}-a} \\
& \times t_{1}^{\epsilon_{2}-1}\left(1-t_{1}\right)^{\epsilon_{3}-1} t_{2}^{\epsilon_{2}-1}\left(1-t_{2}\right)^{\epsilon_{3}-1}\left[\left(1-u_{1}\right)\left(1-v_{2}\right)+u_{1} t_{1}+\left(1-u_{1}\right) v_{2} t_{2}\right]^{-\epsilon_{1}} \\
& \times\left[\left(1-u_{2}\right)\left(1-v_{1}\right)+u_{2}\left(1-t_{1}\right)+\left(1-u_{2}\right) v_{1}\left(1-t_{2}\right)\right]^{-\epsilon_{1}}
\end{aligned}
$$


Next, using Mellin-Barnes integral

$$
\frac{\Gamma(a)}{(A+B+C)^{a}}=\frac{1}{(2 \pi i)^{2}} \int d s_{1} d s_{2} \frac{\Gamma\left(a-s_{1}\right)}{A^{a-s_{1}}} \frac{\Gamma\left(s_{1}-s_{2}\right)}{B^{s_{1}-s_{2}}} \frac{\Gamma\left(s_{2}\right)}{C^{s_{2}}}
$$

one can rewrite eq. (D.6) as follows

$$
\bar{\Lambda}^{a}\left(\epsilon_{1}, \epsilon_{2}, \epsilon_{3}\right)=-\frac{\sin \pi\left(a+\epsilon_{2}\right) \sin \pi\left(\epsilon_{2}\right) \sin \pi\left(\epsilon_{3}-a\right) \sin \pi\left(\epsilon_{3}\right)}{\pi^{3} \sin \pi\left(\epsilon_{2}+\epsilon_{3}\right) \Gamma^{2}\left(\epsilon_{1}\right)} I^{a}\left(\epsilon_{1}, \epsilon_{2}, \epsilon_{3}\right)
$$

where $I^{a}\left(\epsilon_{1}, \epsilon_{2}, \epsilon_{3}\right)=I^{a, a, a, a}\left(\epsilon_{1}, \epsilon_{2}, \epsilon_{3}\right)$ and

$$
\begin{aligned}
& I^{a_{1}, a_{2}, a_{3}, a_{4}}\left(\epsilon_{1}, \epsilon_{2}, \epsilon_{3}\right) \\
& =\int_{\delta-i \infty}^{\delta+i \infty} \frac{d s_{1} d s_{2} d s_{3} d s_{4}}{(2 \pi i)^{4}} \Gamma\left(\epsilon_{1}-s_{1}-s_{2}\right) \Gamma\left(s_{1}\right) \Gamma\left(s_{2}\right) \Gamma\left(\epsilon_{1}-s_{3}-s_{4}\right) \Gamma\left(s_{3}\right) \Gamma\left(s_{4}\right) \\
& \quad \times \frac{\Gamma\left(\epsilon_{2}-1+a_{3}-s_{1}\right) \Gamma\left(s_{1}-a_{3}+1\right)}{\Gamma\left(\epsilon_{2}\right)} \frac{\Gamma\left(1+\epsilon_{3}-a_{1}-s_{3}\right) \Gamma\left(a_{1}-1+s_{3}\right)}{\Gamma\left(\epsilon_{3}\right)} \\
& \quad \times \frac{\Gamma\left(\epsilon_{2}-1+a_{2}-s_{4}\right) \Gamma\left(1-\epsilon_{1}-\epsilon_{2}+s_{3}+s_{4}\right)}{\Gamma\left(a_{2}-\epsilon_{1}+s_{3}\right)} \\
& \quad \times \frac{\Gamma\left(1+\epsilon_{3}-a_{4}-s_{2}\right) \Gamma\left(1-\epsilon_{1}-\epsilon_{3}+s_{1}+s_{2}\right)}{\Gamma\left(2-a_{4}-\epsilon_{1}+s_{1}\right)} \frac{\Gamma\left(\epsilon_{2}-s_{1}\right) \Gamma\left(\epsilon_{3}-s_{3}\right)}{\Gamma\left(\epsilon_{2}+\epsilon_{3}-s_{1}-s_{3}\right)} \frac{\Gamma\left(\epsilon_{2}-s_{2}\right) \Gamma\left(\epsilon_{3}-s_{4}\right)}{\Gamma\left(\epsilon_{2}+\epsilon_{3}-s_{2}-s_{4}\right)}
\end{aligned}
$$

Here we assume $\left|1-a_{i}\right|<\delta<\frac{\epsilon_{i}}{2}$, then the MB integral is well-defined with all the "left" poles of the type $\Gamma\left(s_{i}+\ldots\right)$ to the left of the contour of integration over $s_{i}$ and "right" poles $\sim \Gamma\left(\ldots-s_{i}\right)$ to the right of the contour.

Next, we need to continue analytically to $a_{i}=0$. We will do this separately for each $a_{i}$ paying attention to the poles which intersect the contour of integration and taking residues in those poles as explained in the book [50]. First, note that analytic continuation in $a_{4}$ is trivial: "right" pole at $s_{2}=1+\epsilon_{3}-a_{4}$ moves to the right and away from the contour. Thus, we set $a_{4}=0$ in what follows. At a next step, we continue $a_{1}$ to $a_{1}=0$. There are two poles affected by that: pole at $s_{3}=1+\epsilon_{3}-a_{1}$ and pole at $s_{3}=1-a_{1}$. While the first pole is always to the right of the contour, the second pole intersects the contour so we need to take a residue at $s_{3}=1-a_{1}$. The integral $I^{a_{1}, a_{2}, a_{3}, 0}$ at $a_{1} \leq 1-\delta$ takes the form

$$
\begin{aligned}
& I^{a_{1}, a_{2}, a_{3}, 0}=\text { r.h.s. of eq. (D.9) }\left.\right|_{a_{1}=a_{4}=0} \\
& \quad+\Gamma\left(1-a_{1}\right) \int_{\delta-i \infty}^{\delta+i \infty} \frac{d s_{1} d s_{2} d s_{4}}{(2 \pi i)^{3}} \Gamma\left(\epsilon_{1}-s_{1}-s_{2}\right) \Gamma\left(s_{1}\right) \Gamma\left(s_{2}\right) \Gamma\left(a_{1}+\epsilon_{1}-1-s_{4}\right) \Gamma\left(s_{4}\right) \\
& \quad \times \frac{\Gamma\left(\epsilon_{2}-1+a_{3}-s_{1}\right) \Gamma\left(s_{1}-a_{3}+1\right)}{\Gamma\left(\epsilon_{2}\right)} \frac{\Gamma\left(\epsilon_{2}-1+a_{2}-s_{4}\right) \Gamma\left(2-a_{1}-\epsilon_{1}-\epsilon_{2}+s_{4}\right)}{\Gamma\left(1-a_{1}+a_{2}-\epsilon_{1}\right)} \\
& \quad \times \frac{\Gamma\left(1+\epsilon_{3}-s_{2}\right) \Gamma\left(1-\epsilon_{1}-\epsilon_{3}+s_{1}+s_{2}\right)}{\Gamma\left(2-\epsilon_{1}+s_{1}\right)} \frac{\Gamma\left(\epsilon_{2}-s_{1}\right) \Gamma\left(a_{1}+\epsilon_{3}-1\right)}{\Gamma\left(a_{1}+\epsilon_{2}+\epsilon_{3}-s_{1}-1\right)} \frac{\Gamma\left(\epsilon_{2}-s_{2}\right) \Gamma\left(\epsilon_{3}-s_{4}\right)}{\Gamma\left(\epsilon_{2}+\epsilon_{3}-s_{2}-s_{4}\right)}
\end{aligned}
$$

Now we should continue from $a_{1}=1-\delta$ to $a_{1}=0$. In the first term in r.h.s. of eq. (D.10) there are no more crossings of the contour so we can just set $a_{1}=0$. In the second term, the pole at $s_{4}=a_{1}+\epsilon_{1}+\epsilon_{2}-2$ will always stay to the left of the cut but the pole at 
$s_{4}=a_{1}+\epsilon_{1}-1$ will move from $s_{4}=\epsilon_{1}-\delta$ to $s_{4}=\epsilon_{1}-1$ so it will cross the contour and we need to take the residue. The residue yields

$$
\begin{aligned}
\Gamma\left(1-a_{1}\right) \int_{\delta-i \infty}^{\delta+i \infty} \frac{d s_{1} d s_{2}}{(2 \pi i)^{2}} \Gamma\left(\epsilon_{1}-s_{1}-s_{2}\right) \Gamma\left(s_{1}\right) \Gamma\left(s_{2}\right) \Gamma\left(a_{1}+\epsilon_{1}-1\right) \\
\times \frac{\Gamma\left(\epsilon_{2}-1+a_{3}-s_{1}\right) \Gamma\left(s_{1}-a_{3}+1\right)}{\Gamma\left(\epsilon_{2}\right)} \frac{\Gamma\left(\epsilon_{2}-\epsilon_{1}+a_{2}-a_{1}\right) \Gamma\left(2-a_{1}-\epsilon_{1}-\epsilon_{2}+s_{4}\right)}{\Gamma\left(1-a_{1}+a_{2}-\epsilon_{1}\right)} \\
\times \frac{\Gamma\left(1+\epsilon_{3}-s_{2}\right) \Gamma\left(1-\epsilon_{1}-\epsilon_{3}+s_{1}+s_{2}\right)}{\Gamma\left(2-\epsilon_{1}+s_{1}\right)} \frac{\Gamma\left(\epsilon_{2}-s_{1}\right) \Gamma\left(a_{1}+\epsilon_{3}-1\right)}{\Gamma\left(a_{1}+\epsilon_{2}+\epsilon_{3}-s_{1}-1\right)} \\
\times \frac{\Gamma\left(\epsilon_{2}-s_{2}\right) \Gamma\left(1-\epsilon_{1}-\epsilon_{3}-a_{1}\right)}{\Gamma\left(1+\epsilon_{2}+\epsilon_{3}-\epsilon_{1}-s_{2}-a_{1}\right)}
\end{aligned}
$$

The continuation $a_{1} \rightarrow 0$ in eq. (D.11) does not cross the integration contours so we can set $a_{1}=0$ and get for eq. (D.9)

$$
\begin{aligned}
& I^{a_{1} \rightarrow 0, a_{2}, a_{3}, 0}\left(\epsilon_{1}, \epsilon_{2}, \epsilon_{3}\right) \\
& =\int_{\delta-i \infty}^{\delta+i \infty} \frac{d s_{1} d s_{2} d s_{3} d s_{4}}{(2 \pi i)^{4}} \Gamma\left(\epsilon_{1}-s_{1}-s_{2}\right) \Gamma\left(s_{1}\right) \Gamma\left(s_{2}\right) \Gamma\left(\epsilon_{1}-s_{3}-s_{4}\right) \Gamma\left(s_{3}\right) \Gamma\left(s_{4}\right) \\
& \times \frac{\Gamma\left(\epsilon_{2}-1+a_{3}-s_{1}\right) \Gamma\left(s_{1}-a_{3}+1\right)}{\Gamma\left(\epsilon_{2}\right)} \frac{\Gamma\left(1+\epsilon_{3}-s_{3}\right) \Gamma\left(s_{3}-1\right)}{\Gamma\left(\epsilon_{3}\right)} \\
& \times \frac{\Gamma\left(\epsilon_{2}-1+a_{2}-s_{4}\right) \Gamma\left(1-\epsilon_{1}-\epsilon_{2}+s_{3}+s_{4}\right)}{\Gamma\left(a_{2}-\epsilon_{1}+s_{3}\right)} \\
& \times \frac{\Gamma\left(1+\epsilon_{3}-s_{2}\right) \Gamma\left(1-\epsilon_{1}-\epsilon_{3}+s_{1}+s_{2}\right)}{\Gamma\left(2-\epsilon_{1}+s_{1}\right)} \frac{\Gamma\left(\epsilon_{2}-s_{1}\right) \Gamma\left(\epsilon_{3}-s_{3}\right)}{\Gamma\left(\epsilon_{2}+\epsilon_{3}-s_{1}-s_{3}\right)} \frac{\Gamma\left(\epsilon_{2}-s_{2}\right) \Gamma\left(\epsilon_{3}-s_{4}\right)}{\Gamma\left(\epsilon_{2}+\epsilon_{3}-s_{2}-s_{4}\right)} \\
& +\int_{\delta-i \infty}^{\delta+i \infty} \frac{d s_{1} d s_{2} d s_{4}}{(2 \pi i)^{3}} \Gamma\left(\epsilon_{1}-s_{1}-s_{2}\right) \Gamma\left(s_{1}\right) \Gamma\left(s_{2}\right) \Gamma\left(\epsilon_{1}-1-s_{4}\right) \Gamma\left(s_{4}\right) \\
& \times \frac{\Gamma\left(\epsilon_{2}-1+a_{3}-s_{1}\right) \Gamma\left(s_{1}-a_{3}+1\right)}{\Gamma\left(\epsilon_{2}\right)} \frac{\Gamma\left(\epsilon_{2}-1+a_{2}-s_{4}\right) \Gamma\left(2-\epsilon_{1}-\epsilon_{2}+s_{4}\right)}{\Gamma\left(1+a_{2}-\epsilon_{1}\right)} \\
& \times \frac{\Gamma\left(1+\epsilon_{3}-s_{2}\right) \Gamma\left(1-\epsilon_{1}-\epsilon_{3}+s_{1}+s_{2}\right)}{\Gamma\left(2-\epsilon_{1}+s_{1}\right)} \frac{\Gamma\left(\epsilon_{2}-s_{1}\right) \Gamma\left(\epsilon_{3}-1\right)}{\Gamma\left(\epsilon_{2}+\epsilon_{3}-s_{1}-1\right)} \frac{\Gamma\left(\epsilon_{2}-s_{2}\right) \Gamma\left(\epsilon_{3}-s_{4}\right)}{\Gamma\left(\epsilon_{2}+\epsilon_{3}-s_{2}-s_{4}\right)} \\
& +\int_{\delta-i \infty}^{\delta+i \infty} \frac{d s_{1} d s_{2}}{(2 \pi i)^{2}} \Gamma\left(\epsilon_{1}-s_{1}-s_{2}\right) \Gamma\left(s_{1}\right) \Gamma\left(s_{2}\right) \Gamma\left(\epsilon_{1}-1\right) \\
& \times \frac{\Gamma\left(\epsilon_{2}-1+a_{3}-s_{1}\right) \Gamma\left(s_{1}-a_{3}+1\right)}{\Gamma\left(\epsilon_{2}\right)} \frac{\Gamma\left(\epsilon_{2}-\epsilon_{1}+a_{2}\right) \Gamma\left(2-\epsilon_{1}-\epsilon_{2}+s_{4}\right)}{\Gamma\left(1+a_{2}-\epsilon_{1}\right)} \\
& \times \frac{\Gamma\left(1+\epsilon_{3}-s_{2}\right) \Gamma\left(1-\epsilon_{1}-\epsilon_{3}+s_{1}+s_{2}\right)}{\Gamma\left(2-\epsilon_{1}+s_{1}\right)} \frac{\Gamma\left(\epsilon_{2}-s_{1}\right) \Gamma\left(\epsilon_{3}-1\right)}{\Gamma\left(\epsilon_{2}+\epsilon_{3}-s_{1}-1\right)} \frac{\Gamma\left(\epsilon_{2}-s_{2}\right) \Gamma\left(1-\epsilon_{1}-\epsilon_{3}\right)}{\Gamma\left(1+\epsilon_{2}+\epsilon_{3}-\epsilon_{1}-s_{2}\right)}
\end{aligned}
$$

Repeating this procedure for $a_{2}$ and $a_{3}$ one obtains after some algebra

$$
\begin{aligned}
\bar{\Lambda}\left(\epsilon_{1}, \epsilon_{2}, \epsilon_{3}\right) & =-\frac{\sin ^{2} \pi \epsilon_{2} \sin ^{2} \pi \epsilon_{3}}{\pi^{3} \sin \pi\left(\epsilon_{2}+\epsilon_{3}\right) \Gamma^{2}\left(\epsilon_{1}\right)} I^{a_{1} \rightarrow 0, a_{2} \rightarrow 0, a_{3} \rightarrow 0,0}\left(\epsilon_{1}, \epsilon_{2}, \epsilon_{3}\right) \\
I^{a_{1} \rightarrow 0, a_{2} \rightarrow 0, a_{3} \rightarrow 0,0}\left(\epsilon_{1}, \epsilon_{2}, \epsilon_{3}\right) & =J_{1}\left(\epsilon_{i}\right)+J_{2}\left(\epsilon_{i}\right)+\ldots+J_{14}\left(\epsilon_{i}\right)
\end{aligned}
$$


where

$$
\begin{aligned}
& J_{1}=\int_{\delta-i \infty}^{\delta+i \infty} \frac{d s_{1} d s_{2} d s_{3} d s_{4}}{(2 \pi i)^{4}} \Gamma\left(\epsilon_{1}-s_{1}-s_{2}\right) \Gamma\left(s_{1}\right) \Gamma\left(s_{2}\right) \Gamma\left(\epsilon_{1}-s_{3}-s_{4}\right) \Gamma\left(s_{3}\right) \Gamma\left(s_{4}\right) \\
& \times \frac{\Gamma\left(\epsilon_{2}-1-s_{1}\right) \Gamma\left(s_{1}+1\right)}{\Gamma\left(\epsilon_{2}\right)} \frac{\Gamma\left(1+\epsilon_{3}-s_{3}\right) \Gamma\left(s_{3}-1\right)}{\Gamma\left(\epsilon_{3}\right)} \frac{\Gamma\left(\epsilon_{2}-1-s_{4}\right) \Gamma\left(1-\epsilon_{1}-\epsilon_{2}+s_{3}+s_{4}\right)}{\Gamma\left(-\epsilon_{1}+s_{3}\right)} \\
& \times \frac{\Gamma\left(1+\epsilon_{3}-s_{2}\right) \Gamma\left(1-\epsilon_{1}-\epsilon_{3}+s_{1}+s_{2}\right)}{\Gamma\left(2-\epsilon_{1}+s_{1}\right)} \frac{\Gamma\left(\epsilon_{2}-s_{1}\right) \Gamma\left(\epsilon_{3}-s_{3}\right)}{\Gamma\left(\epsilon_{2}+\epsilon_{3}-s_{1}-s_{3}\right)} \frac{\Gamma\left(\epsilon_{2}-s_{2}\right) \Gamma\left(\epsilon_{3}-s_{4}\right)}{\Gamma\left(\epsilon_{2}+\epsilon_{3}-s_{2}-s_{4}\right)} \\
& J_{2}=\frac{\Gamma\left(\epsilon_{2}-1\right) \Gamma\left(1+\epsilon_{3}-\epsilon_{2}\right)}{\Gamma\left(\epsilon_{2}\right) \Gamma\left(\epsilon_{3}\right)} \int_{\delta-i \infty}^{\delta+i \infty} \frac{d s_{1} d s_{2} d s_{3}}{(2 \pi i)^{3}} \Gamma\left(\epsilon_{1}-s_{1}-s_{2}\right) \Gamma\left(s_{1}\right) \Gamma\left(s_{2}\right) \\
& \times \Gamma\left(1+\epsilon_{1}-\epsilon_{2}-s_{3}\right) \Gamma\left(s_{3}\right) \Gamma\left(\epsilon_{2}-1-s_{1}\right) \Gamma\left(s_{1}+1\right) \Gamma\left(1+\epsilon_{3}-s_{3}\right) \Gamma\left(s_{3}-1\right) \\
& \times \frac{\Gamma\left(1+\epsilon_{3}-s_{2}\right) \Gamma\left(1-\epsilon_{1}-\epsilon_{3}+s_{1}+s_{2}\right)}{\Gamma\left(2-\epsilon_{1}+s_{1}\right)} \frac{\Gamma\left(\epsilon_{2}-s_{1}\right) \Gamma\left(\epsilon_{3}-s_{3}\right)}{\Gamma\left(\epsilon_{2}+\epsilon_{3}-s_{1}-s_{3}\right)} \frac{\Gamma\left(\epsilon_{2}-s_{2}\right)}{\Gamma\left(1+\epsilon_{3}-s_{2}\right)} \\
& J_{3}=\frac{\Gamma\left(\epsilon_{3}-1\right)}{\Gamma\left(\epsilon_{2}\right)} \int_{\delta-i \infty}^{\delta+i \infty} \frac{d s_{1} d s_{2} d s_{4}}{(2 \pi i)^{3}} \Gamma\left(\epsilon_{1}-s_{1}-s_{2}\right) \Gamma\left(s_{1}\right) \Gamma\left(s_{2}\right) \Gamma\left(\epsilon_{1}-1-s_{4}\right) \Gamma\left(s_{4}\right) \\
& \times \Gamma\left(\epsilon_{2}-1-s_{1}\right) \Gamma\left(s_{1}+1\right) \frac{\Gamma\left(\epsilon_{2}-1-s_{4}\right) \Gamma\left(2-\epsilon_{1}-\epsilon_{2}+s_{4}\right)}{\Gamma\left(1-\epsilon_{1}\right)} \frac{\Gamma\left(1+\epsilon_{3}-s_{2}\right)}{\Gamma\left(2-\epsilon_{1}+s_{1}\right)} \\
& \times \Gamma\left(1-\epsilon_{1}-\epsilon_{3}+s_{1}+s_{2}\right) \frac{\Gamma\left(\epsilon_{2}-s_{1}\right)}{\Gamma\left(\epsilon_{2}+\epsilon_{3}-s_{1}-1\right)} \frac{\Gamma\left(\epsilon_{2}-s_{2}\right) \Gamma\left(\epsilon_{3}-s_{4}\right)}{\Gamma\left(\epsilon_{2}+\epsilon_{3}-s_{2}-s_{4}\right)} \\
& J_{4}=\frac{\Gamma\left(\epsilon_{2}-1\right)}{\Gamma\left(\epsilon_{3}\right) \Gamma\left(1-\epsilon_{1}+\epsilon_{2}\right)} \int_{\delta-i \infty}^{\delta+i \infty} \frac{d s_{2} d s_{3} d s_{4}}{(2 \pi i)^{3}} \Gamma\left(1+\epsilon_{1}-\epsilon_{2}-s_{2}\right) \Gamma\left(s_{2}\right) \Gamma\left(\epsilon_{1}-s_{3}-s_{4}\right) \\
& \times \Gamma\left(s_{3}\right) \Gamma\left(s_{4}\right) \Gamma\left(1+\epsilon_{3}-s_{3}\right) \Gamma\left(s_{3}-1\right) \frac{\Gamma\left(\epsilon_{2}-1-s_{4}\right) \Gamma\left(1-\epsilon_{1}-\epsilon_{2}+s_{3}+s_{4}\right)}{\left(\epsilon_{3}-s_{3}\right) \Gamma\left(-\epsilon_{1}+s_{3}\right)} \\
& \times \Gamma\left(1+\epsilon_{3}-s_{2}\right) \Gamma\left(\epsilon_{2}-\epsilon_{1}-\epsilon_{3}+s_{2}\right) \frac{\Gamma\left(\epsilon_{2}-s_{2}\right) \Gamma\left(\epsilon_{3}-s_{4}\right)}{\Gamma\left(\epsilon_{2}+\epsilon_{3}-s_{2}-s_{4}\right)} \\
& J_{5}=-\frac{\Gamma\left(\epsilon_{1}-\epsilon_{2}\right)}{\left(1-\epsilon_{2}\right)} \Gamma\left(\epsilon_{3}-1\right) \Gamma\left(1+\epsilon_{3}-\epsilon_{2}\right) \int_{\delta-i \infty}^{\delta+i \infty} \frac{d s_{1} d s_{2}}{(2 \pi i)^{2}} \Gamma\left(\epsilon_{1}-s_{1}-s_{2}\right) \Gamma\left(s_{1}\right) \Gamma\left(s_{2}\right) \\
& \times \Gamma\left(\epsilon_{2}-1-s_{1}\right) \Gamma\left(s_{1}+1\right) \frac{\Gamma\left(1+\epsilon_{3}-s_{2}\right) \Gamma\left(1-\epsilon_{1}-\epsilon_{3}+s_{1}+s_{2}\right) \Gamma\left(\epsilon_{2}-s_{1}\right) \Gamma\left(\epsilon_{2}-s_{2}\right)}{\Gamma\left(2-\epsilon_{1}+s_{1}\right) \Gamma\left(\epsilon_{2}+\epsilon_{3}-s_{1}-1\right) \Gamma\left(1+\epsilon_{3}-s_{2}\right)} \\
& J_{6}=\frac{\Gamma\left(\epsilon_{2}-\epsilon_{1}\right) \Gamma\left(1-\epsilon_{2}\right) \Gamma\left(1+\epsilon_{3}-\epsilon_{1}\right)}{\Gamma\left(\epsilon_{2}\right) \Gamma\left(1-\epsilon_{1}\right)} \Gamma\left(\epsilon_{1}-1\right) \Gamma\left(\epsilon_{3}-1\right) \\
& \times \int_{\delta-i \infty}^{\delta+i \infty} \frac{d s_{1} d s_{2}}{(2 \pi i)^{2}} \Gamma\left(\epsilon_{1}-s_{1}-s_{2}\right) \Gamma\left(s_{1}\right) \Gamma\left(s_{2}\right) \Gamma\left(\epsilon_{2}-1-s_{1}\right) \Gamma\left(s_{1}+1\right) \\
& \times \frac{\Gamma\left(1+\epsilon_{3}-s_{2}\right) \Gamma\left(1-\epsilon_{1}-\epsilon_{3}+s_{1}+s_{2}\right) \Gamma\left(\epsilon_{2}-s_{1}\right) \Gamma\left(\epsilon_{2}-s_{2}\right)}{\Gamma\left(2-\epsilon_{1}+s_{1}\right) \Gamma\left(\epsilon_{2}+\epsilon_{3}-s_{1}-1\right) \Gamma\left(1+\epsilon_{2}+\epsilon_{3}-\epsilon_{1}-s_{2}\right)} \\
& J_{7}=\frac{\Gamma^{2}\left(\epsilon_{2}-1\right) \Gamma\left(1+\epsilon_{3}-\epsilon_{2}\right)}{\Gamma\left(\epsilon_{3}\right) \Gamma\left(1+\epsilon_{2}-\epsilon_{1}\right)} \int_{\delta-i \infty}^{\delta+i \infty} \frac{d s_{2} d s_{3}}{(2 \pi i)^{2}} \Gamma\left(1+\epsilon_{1}-\epsilon_{2}-s_{2}\right) \\
& \times \Gamma\left(s_{2}\right) \Gamma\left(1+\epsilon_{1}-\epsilon_{2}-s_{3}\right) \Gamma\left(s_{3}\right) \Gamma\left(1+\epsilon_{3}-s_{3}\right) \Gamma\left(s_{3}-1\right) \\
& \times \Gamma\left(1+\epsilon_{3}-s_{2}\right) \Gamma\left(\epsilon_{2}-\epsilon_{1}-\epsilon_{3}+s_{2}\right) \frac{\Gamma\left(\epsilon_{3}-s_{3}\right)}{\Gamma\left(1+\epsilon_{3}-s_{3}\right)} \frac{\Gamma\left(\epsilon_{2}-s_{2}\right)}{\Gamma\left(1+\epsilon_{3}-s_{2}\right)}
\end{aligned}
$$




$$
\begin{aligned}
& J_{8}=-\int_{\delta-i \infty}^{\delta+i \infty} \frac{d s_{2} d s_{4}}{(2 \pi i)^{2}} \Gamma\left(1+\epsilon_{1}-\epsilon_{2}-s_{2}\right) \\
& \times \Gamma\left(\epsilon_{2}-1\right) \Gamma\left(s_{2}\right) \Gamma\left(\epsilon_{1}-1-s_{4}\right) \Gamma\left(s_{4}\right) \frac{\Gamma\left(\epsilon_{2}-1-s_{4}\right) \Gamma\left(2-\epsilon_{1}-\epsilon_{2}+s_{4}\right)}{\left(1-\epsilon_{3}\right) \Gamma\left(1-\epsilon_{1}\right)} \\
& \times \frac{\Gamma\left(1+\epsilon_{3}-s_{2}\right) \Gamma\left(\epsilon_{2}-\epsilon_{1}-\epsilon_{3}+s_{2}\right)}{\Gamma\left(2-\epsilon_{1}+s_{1}\right)} \frac{\Gamma\left(\epsilon_{2}-s_{2}\right) \Gamma\left(\epsilon_{3}-s_{4}\right)}{\Gamma\left(\epsilon_{2}+\epsilon_{3}-s_{2}-s_{4}\right)} \\
& J_{9}=\frac{\Gamma\left(\epsilon_{2}-1\right) \Gamma\left(\epsilon_{1}+\epsilon_{3}-\epsilon_{2}\right)}{\Gamma\left(\epsilon_{3}\right)} \Gamma\left(2 \epsilon_{2}-\epsilon_{1}-\epsilon_{3}\right) \int_{\delta-i \infty}^{\delta+i \infty} \frac{d s_{3} d s_{4}}{(2 \pi i)^{2}} \Gamma\left(\epsilon_{1}-s_{3}-s_{4}\right) \Gamma\left(s_{3}\right) \\
& \times \Gamma\left(s_{4}\right) \Gamma\left(\epsilon_{3}-s_{3}\right) \Gamma\left(s_{3}-1\right) \frac{\Gamma\left(\epsilon_{2}-1-s_{4}\right) \Gamma\left(1-\epsilon_{1}-\epsilon_{2}+s_{3}+s_{4}\right) \Gamma\left(\epsilon_{3}-s_{4}\right)}{\Gamma\left(-\epsilon_{1}+s_{3}\right) \Gamma\left(2 \epsilon_{2}-\epsilon_{1}-s_{4}\right)} \\
& J_{10}=-\frac{\Gamma^{2}\left(\epsilon_{2}-1\right) \Gamma\left(\epsilon_{1}-\epsilon_{2}\right)}{\left(1-\epsilon_{3}\right) \Gamma\left(1+\epsilon_{2}-\epsilon_{1}\right)} \Gamma\left(1+\epsilon_{3}-\epsilon_{2}\right) \int_{\delta-i \infty}^{\delta+i \infty} \frac{d s_{2}}{2 \pi i} \Gamma\left(1+\epsilon_{1}-\epsilon_{2}-s_{2}\right) \Gamma\left(s_{2}\right) \\
& \times \Gamma\left(1+\epsilon_{3}-s_{2}\right) \Gamma\left(\epsilon_{2}-\epsilon_{1}-\epsilon_{3}+s_{2}\right) \frac{\Gamma\left(\epsilon_{2}-s_{2}\right)}{\Gamma\left(1+\epsilon_{3}-s_{2}\right)} \\
& J_{11}=-\Gamma\left(\epsilon_{1}-1\right) \Gamma\left(\epsilon_{2}-1\right) \frac{\Gamma\left(\epsilon_{2}-\epsilon_{1}\right) \Gamma\left(1-\epsilon_{2}\right)}{\left(1-\epsilon_{3}\right) \Gamma\left(1-\epsilon_{1}\right)} \int_{\delta-i \infty}^{\delta+i \infty} \frac{d s_{2}}{2 \pi i} \Gamma\left(1+\epsilon_{1}-\epsilon_{2}-s_{2}\right) \Gamma\left(s_{2}\right) \\
& \times \frac{\Gamma\left(1+\epsilon_{3}-s_{2}\right) \Gamma\left(\epsilon_{2}-\epsilon_{1}-\epsilon_{3}+s_{2}\right)}{\Gamma\left(1+\epsilon_{2}-\epsilon_{1}\right)} \frac{\Gamma\left(\epsilon_{2}-s_{2}\right) \Gamma\left(1+\epsilon_{3}-\epsilon_{1}\right)}{\Gamma\left(1+\epsilon_{2}+\epsilon_{3}-\epsilon_{1}-s_{2}\right)} \\
& J_{12}=\Gamma^{2}\left(\epsilon_{2}-1\right) \Gamma\left(\epsilon_{1}+\epsilon_{3}-\epsilon_{2}\right) \frac{\Gamma\left(2 \epsilon_{2}-\epsilon_{1}-\epsilon_{3}\right) \Gamma\left(1+\epsilon_{3}-\epsilon_{2}\right)}{\Gamma\left(\epsilon_{3}\right) \Gamma\left(1-\epsilon_{1}+\epsilon_{2}\right)} \\
& \times \int_{\delta-i \infty}^{\delta+i \infty} \frac{d s_{3}}{2 \pi i} \Gamma\left(1+\epsilon_{1}-\epsilon_{2}-s_{3}\right) \Gamma\left(s_{3}\right) \Gamma\left(1+\epsilon_{3}-s_{3}\right) \Gamma\left(s_{3}-1\right) \frac{\Gamma\left(\epsilon_{3}-s_{3}\right)}{\Gamma\left(1+\epsilon_{3}-s_{3}\right)} \\
& J_{13}=-\frac{\Gamma\left(1-\epsilon_{3}\right) \Gamma\left(\epsilon_{2}-1\right) \Gamma\left(\epsilon_{1}+\epsilon_{3}-\epsilon_{2}\right)}{\left(1-\epsilon_{3}\right) \Gamma\left(1-\epsilon_{1}\right)} \int_{\delta-i \infty}^{\delta+i \infty} \frac{d s_{4}}{2 \pi i} \Gamma\left(\epsilon_{1}-1-s_{4}\right) \Gamma\left(s_{4}\right) \\
& \times \Gamma\left(\epsilon_{2}-1-s_{4}\right) \Gamma\left(2-\epsilon_{1}-\epsilon_{2}+s_{4}\right) \frac{\Gamma\left(2 \epsilon_{2}-\epsilon_{1}-\epsilon_{3}\right) \Gamma\left(\epsilon_{3}-s_{4}\right)}{\Gamma\left(2 \epsilon_{2}-\epsilon_{1}-s_{4}\right)} \\
& J_{14}=-\Gamma\left(1-\epsilon_{3}\right) \Gamma^{2}\left(\epsilon_{2}-1\right) \Gamma\left(\epsilon_{1}+\epsilon_{3}-\epsilon_{2}\right) \Gamma\left(\epsilon_{1}-\epsilon_{2}\right) \frac{\left.\Gamma\left(2 \epsilon_{2}-\epsilon_{1}-\epsilon_{3}\right)\right) \Gamma\left(1+\epsilon_{3}-\epsilon_{2}\right)}{\left(1-\epsilon_{3}\right) \Gamma\left(1+\epsilon_{2}-\epsilon_{1}\right)} \\
& J_{15}=-\Gamma\left(1-\epsilon_{3}\right) \Gamma\left(\epsilon_{2}-1\right) \Gamma\left(\epsilon_{1}+\epsilon_{3}-\epsilon_{2}\right) \Gamma\left(\epsilon_{1}-1\right) \\
& \times \frac{\Gamma\left(\epsilon_{2}-\epsilon_{1}\right) \Gamma\left(1-\epsilon_{2}\right)}{\Gamma\left(1-\epsilon_{1}\right)} \frac{\Gamma\left(2 \epsilon_{2}-\epsilon_{1}-\epsilon_{3}\right) \Gamma\left(1+\epsilon_{3}-\epsilon_{1}\right)}{\left(1-\epsilon_{3}\right) \Gamma\left(1+2 \epsilon_{2}-2 \epsilon_{1}\right)}
\end{aligned}
$$

The combination of eq. (D.13) and (D.14) is the final result for the function $\tilde{\Lambda}\left(\gamma_{i}\right)$ (our notation is $\gamma_{i}=-2 \epsilon_{i}$ ). Unfortunately, I was not able to find a representation of the sum (D.13) which would be explicitly symmetric in $\epsilon_{1}, \epsilon_{2}$, and $\epsilon_{3}$. However, the result (D.34) in the limit $\epsilon_{i} \rightarrow 0$ obtained below is symmetric.

To get $\bar{\Lambda}\left(\epsilon_{i}\right)$ at small $\epsilon_{i}$ we need to estimate the behavior of the integrals $J_{1^{-}} J_{13}$ as $\epsilon_{i} \rightarrow 0$. As an example, let us consider integral $J_{9}$ given by eq. (D.23). The contours of integration over $s_{3}$ and $s_{4}$ are pinched between "left" and "right" poles as the separation between them vanishes in the limit $\epsilon_{i} \rightarrow 0$. Shifting contours of integration over $s_{3}$ and $s_{4}$ 
to the left of the real axis and taking residues at $s_{3}=0$ and $s_{4}=0$ one obtains

$$
\begin{aligned}
J_{9}= & \frac{\Gamma\left(\epsilon_{2}-1\right) \Gamma\left(\epsilon_{1}+\epsilon_{3}-\epsilon_{2}\right)}{\Gamma\left(\epsilon_{3}\right)} \Gamma\left(2 \epsilon_{2}-\epsilon_{1}-\epsilon_{3}\right) \int_{-\delta-i \infty}^{-\delta+i \infty} \frac{d s_{3} d s_{4}}{(2 \pi i)^{2}} \Gamma\left(\epsilon_{1}-s_{3}-s_{4}\right) \Gamma\left(s_{3}\right) \\
& \times \Gamma\left(s_{4}\right) \Gamma\left(\epsilon_{3}-s_{3}\right) \Gamma\left(s_{3}-1\right) \frac{\Gamma\left(\epsilon_{2}-1-s_{4}\right) \Gamma\left(1-\epsilon_{1}-\epsilon_{2}+s_{3}+s_{4}\right) \Gamma\left(\epsilon_{3}-s_{4}\right)}{\Gamma\left(-\epsilon_{1}+s_{3}\right) \Gamma\left(2 \epsilon_{2}-\epsilon_{1}-s_{4}\right)} \\
+ & \frac{\Gamma^{2}\left(\epsilon_{2}-1\right) \Gamma\left(\epsilon_{1}+\epsilon_{3}-\epsilon_{2}\right)}{\Gamma\left(2 \epsilon_{2}-\epsilon_{1}\right)} \Gamma\left(2 \epsilon_{2}-\epsilon_{1}-\epsilon_{3}\right) \\
& \times \int_{-\delta-i \infty}^{-\delta+i \infty} \frac{d s_{3}}{2 \pi i} \Gamma\left(\epsilon_{1}-s_{3}\right) \Gamma\left(s_{3}\right) \Gamma\left(\epsilon_{3}-s_{3}\right) \Gamma\left(s_{3}-1\right) \frac{\Gamma\left(1-\epsilon_{1}-\epsilon_{2}+s_{3}\right)}{\Gamma\left(-\epsilon_{1}+s_{3}\right)} \\
+ & \frac{\Gamma\left(\epsilon_{2}-1\right)}{\Gamma\left(-\epsilon_{1}\right)} \Gamma\left(2 \epsilon_{2}-\epsilon_{1}-\epsilon_{3}\right) \Gamma\left(\epsilon_{1}+\epsilon_{3}-\epsilon_{2}\right) \\
& \times \int_{-\delta-i \infty}^{-\delta+i \infty} \frac{d s_{4}}{2 \pi i} \Gamma\left(\epsilon_{1}-s_{4}\right) \Gamma\left(s_{4}\right) \Gamma\left(\epsilon_{2}-1-s_{4}\right) \Gamma\left(1-\epsilon_{1}-\epsilon_{2}+s_{4}\right) \frac{\Gamma\left(\epsilon_{3}-s_{4}\right)}{\Gamma\left(2 \epsilon_{2}-\epsilon_{1}-s_{4}\right)} \\
& \times\left[\psi\left(\epsilon_{1}-s_{4}\right)+\psi\left(\epsilon_{3}\right)-\psi\left(1-\epsilon_{1}-\epsilon_{2}+s_{4}\right)+\psi\left(-\epsilon_{1}\right)-2 \psi(1)-1\right] \\
+ & \frac{\Gamma^{2}\left(\epsilon_{2}-1\right)}{\Gamma\left(-\epsilon_{1}\right)} \Gamma\left(2 \epsilon_{2}-\epsilon_{1}-\epsilon_{3}\right) \Gamma\left(\epsilon_{1}+\epsilon_{3}-\epsilon_{2}\right) \Gamma\left(\epsilon_{1}\right) \Gamma\left(1-\epsilon_{1}-\epsilon_{2}\right) \frac{\Gamma\left(\epsilon_{3}\right)}{\Gamma\left(2 \epsilon_{2}-\epsilon_{1}\right)} \\
& \times\left[\psi\left(\epsilon_{1}\right)+\psi\left(\epsilon_{3}\right)-\psi\left(1-\epsilon_{1}-\epsilon_{2}\right)+\psi\left(-\epsilon_{1}\right)-2 \psi(1)-1\right]
\end{aligned}
$$

where $\psi(x) \equiv \Gamma^{\prime}(x) / \Gamma(x)$. Now the integrals over $s_{3}$ and/or $s_{4}$ in the r.h.s. of eq. (D.30) are not pinched so the only singularities at $\epsilon_{i} \rightarrow 0$ come from the explicit factors like $\Gamma\left(\epsilon_{i}\right)$ or $\psi\left(\epsilon_{i}\right)$. Actually, it is easy to see that the last non-integral term is the most singular so one obtains

$$
J_{9} \stackrel{\epsilon_{i} \rightarrow 0}{\simeq} \frac{2 \epsilon_{2}-\epsilon_{1}}{\epsilon_{2}^{2} \epsilon_{3}^{2}\left(\epsilon_{1}+\epsilon_{3}-\epsilon_{2}\right)\left(2 \epsilon_{2}-\epsilon_{1}-\epsilon_{3}\right)}+O\left(\frac{1}{\epsilon^{4}}\right)
$$

Similar estimates of remaining integrals yield

$$
\begin{array}{rlrl}
J_{1} & \sim J_{2} \sim O\left(\frac{1}{\epsilon^{4}}\right), \quad J_{3} \simeq \frac{\left(\epsilon_{2}+\epsilon_{3}\right)^{2}}{\epsilon_{1}^{2} \epsilon_{2}^{3} \epsilon_{3}^{2}}, & J_{4} \simeq-\frac{\epsilon_{2}+\epsilon_{3}}{\epsilon_{2}^{3} \epsilon_{3}^{2}\left(\epsilon_{1}+\epsilon_{3}-\epsilon_{2}\right)}, \\
J_{5} \simeq \frac{\epsilon_{2}+\epsilon_{3}}{\epsilon_{1} \epsilon_{2}^{3} \epsilon_{3}\left(\epsilon_{1}-\epsilon_{2}\right)}, \quad J_{6} \simeq-\frac{\epsilon_{2}+\epsilon_{3}}{\epsilon_{1}^{2} \epsilon_{2}^{2} \epsilon_{3}\left(\epsilon_{1}-\epsilon_{2}\right)}, & J_{7} \simeq \frac{1}{\epsilon_{2}^{3} \epsilon_{3}\left(\epsilon_{1}+\epsilon_{3}-\epsilon_{2}\right)}, \\
J_{8} \simeq-\frac{\epsilon_{2}+\epsilon_{3}}{\epsilon_{1} \epsilon_{2}^{3} \epsilon_{3}\left(\epsilon_{1}+\epsilon_{3}-\epsilon_{2}\right)}, & J_{10} \simeq \frac{1}{\epsilon_{2}^{3}\left(\epsilon_{1}-\epsilon_{2}\right)\left(\epsilon_{1}+\epsilon_{3}-\epsilon_{2}\right)} \\
J_{11} \simeq-\frac{1}{\epsilon_{1} \epsilon_{2}^{2}\left(\epsilon_{1}-\epsilon_{2}\right)\left(\epsilon_{1}+\epsilon_{3}-\epsilon_{2}\right)}, & J_{12} \simeq \frac{-1}{\epsilon_{2}^{2} \epsilon_{3}\left(\epsilon_{1}+\epsilon_{3}-\epsilon_{2}\right)\left(2 \epsilon_{2}-\epsilon_{1}-\epsilon_{3}\right)} \\
J_{13} \simeq \frac{2 \epsilon_{2}-\epsilon_{1}}{\epsilon_{1} \epsilon_{2}^{2} \epsilon_{3}\left(\epsilon_{1}+\epsilon_{3}-\epsilon_{2}\right)\left(2 \epsilon_{2}-\epsilon_{1}-\epsilon_{3}\right)}, & J_{14} \simeq \frac{1}{\epsilon_{2}^{2}\left(2 \epsilon_{2}-\epsilon_{1}-\epsilon_{3}\right)\left(\epsilon_{1}+\epsilon_{3}-\epsilon_{2}\right)\left(\epsilon_{1}-\epsilon_{2}\right)} \\
J_{15} \simeq \frac{1}{\epsilon_{1} \epsilon_{2}\left(2 \epsilon_{2}-\epsilon_{1}-\epsilon_{3}\right)\left(\epsilon_{1}-\epsilon_{2}\right)\left(\epsilon_{1}+\epsilon_{3}-\epsilon_{2}\right)} & &
\end{array}
$$

It is easy to see that

$$
J_{4}+J_{7}+J_{8}+J_{9}+J_{10}+J_{11}+J_{12}+J_{13}+J_{14}+J_{15}=O\left(\frac{1}{\epsilon^{4}}\right)
$$


so we get

$$
I^{a_{1} \rightarrow 0, a_{2} \rightarrow 0, a_{3} \rightarrow 0,0}\left(\epsilon_{1}, \epsilon_{2}, \epsilon_{3}\right) \simeq J_{3}+J_{5}+J_{6} \simeq-\frac{\epsilon_{2}+\epsilon_{3}}{\epsilon_{1}^{2} \epsilon_{2}^{2} \epsilon_{3}^{2}}+O\left(\frac{1}{\epsilon^{4}}\right)
$$

and therefore

$$
\bar{\Lambda}\left(\epsilon_{1}, \epsilon_{2}, \epsilon_{3}\right)=1+O\left(\epsilon_{i}\right)
$$

which is quoted in eq. (6.42) in terms of $\gamma_{i}=-2 \epsilon_{i}$. Note that the symmetric form of this result for $\bar{\Lambda}\left(\epsilon_{1}, \epsilon_{2}, \epsilon_{3}\right)$ is a check for the calculation of the integral (D.1) which is not obviously symmetric in $\epsilon_{1}, \epsilon_{2}, \epsilon_{3}$.

Open Access. This article is distributed under the terms of the Creative Commons Attribution License (CC-BY 4.0), which permits any use, distribution and reproduction in any medium, provided the original author(s) and source are credited.

\section{References}

[1] S. Moch, J.A.M. Vermaseren and A. Vogt, The Three loop splitting functions in QCD: The Nonsinglet case, Nucl. Phys. B 688 (2004) 101 [hep-ph/0403192] [INSPIRE].

[2] A. Vogt, S. Moch and J.A.M. Vermaseren, The Three-loop splitting functions in QCD: The Singlet case, Nucl. Phys. B 691 (2004) 129 [hep-ph/0404111] [InSPIRE].

[3] A. Grozin, J.M. Henn, G.P. Korchemsky and P. Marquard, Three Loop Cusp Anomalous Dimension in QCD, Phys. Rev. Lett. 114 (2015) 062006 [arXiv: 1409.0023] [INSPIRE].

[4] A. Grozin, J.M. Henn, G.P. Korchemsky and P. Marquard, The three-loop cusp anomalous dimension in $Q C D$ and its supersymmetric extensions, JHEP 01 (2016) 140 [arXiv: 1510.07803] [INSPIRE].

[5] N. Gromov, V. Kazakov, S. Leurent and D. Volin, Quantum Spectral Curve for Planar $\mathcal{N}=4$ Super-Yang-Mills Theory, Phys. Rev. Lett. 112 (2014) 011602 [arXiv:1305.1939] [INSPIRE].

[6] N. Gromov, V. Kazakov, S. Leurent and D. Volin, Quantum spectral curve for arbitrary state/operator in $A d S_{5} / C F T_{4}, J H E P 09$ (2015) 187 [arXiv: 1405.4857] [INSPIRE].

[7] C. Marboe and V. Velizhanin, Twist-2 at seven loops in planar $\mathcal{N}=4$ SYM theory: full result and analytic properties, JHEP 11 (2016) 013 [arXiv:1607.06047] [INSPIRE].

[8] N. Gromov, F. Levkovich-Maslyuk, G. Sizov and S. Valatka, Quantum spectral curve at work: from small spin to strong coupling in $\mathcal{N}=4$ SYM, JHEP 07 (2014) 156 [arXiv: 1402.0871] [INSPIRE].

[9] V. Kazakov and E. Sobko, Three-point correlators of twist-2 operators in $N=4$ SYM at Born approximation, JHEP 06 (2013) 061 [arXiv:1212.6563] [INSPIRE].

[10] E. Sobko, A new representation for two- and three-point correlators of operators from sl(2) sector, JHEP 12 (2014) 101 [arXiv:1311.6957] [INSPIRE].

[11] B. Basso, S. Komatsu and P. Vieira, Structure Constants and Integrable Bootstrap in Planar $N=4$ SYM Theory, arXiv: 1505.06745 [INSPIRE].

[12] B. Eden and F. Paul, Half-BPS half-BPS twist two at four loops in $N=4 S Y M$, arXiv: 1608.04222 [INSPIRE]. 
[13] D. Chicherin, A. Georgoudis, V. Gonçalves and R. Pereira, All five-loop planar four-point functions of half-BPS operators in $\mathcal{N}=4$ SYM, JHEP 11 (2018) 069 [arXiv:1809.00551] [INSPIRE].

[14] A. Cavaglià, N. Gromov and F. Levkovich-Maslyuk, Quantum spectral curve and structure constants in $\mathcal{N}=4$ SYM: cusps in the ladder limit, JHEP 10 (2018) 060 [arXiv: 1802.04237] [INSPIRE].

[15] J. Collins, Foundations of perturbative QCD, Cambridge University Press (2013) [InSPIRE].

[16] I.I. Balitsky and V.M. Braun, Evolution Equations for QCD String Operators, Nucl. Phys. B 311 (1989) 541 [INSPIRE].

[17] I. Balitsky, V. Kazakov and E. Sobko, Two-point correlator of twist-2 light-ray operators in $N=4$ SYM in BFKL approximation, arXiv:1310.3752 [INSPIRE].

[18] A.V. Belitsky, S.E. Derkachov, G.P. Korchemsky and A.N. Manashov, Superconformal operators in $N=4$ superYang-Mills theory, Phys. Rev. D 70 (2004) 045021 [hep-th/0311104] [INSPIRE].

[19] V.S. Fadin and L.N. Lipatov, BFKL Pomeron in the next-to-leading approximation, Phys. Lett. B 429 (1998) 127 [hep-ph/9802290] [INSPIRE].

[20] N. Gromov, F. Levkovich-Maslyuk and G. Sizov, Pomeron Eigenvalue at Three Loops in $\mathcal{N}=4$ Supersymmetric Yang-Mills Theory, Phys. Rev. Lett. 115 (2015) 251601 [arXiv: 1507.04010] [INSPIRE].

[21] V.N. Velizhanin, BFKL Pomeron in the next-to-next-to-leading approximation in the planar $N=4 S Y M$ theory, arXiv: 1508.02857 [INSPIRE].

[22] S. Caron-Huot and M. Herranen, High-energy evolution to three loops, JHEP 02 (2018) 058 [arXiv: 1604.07417] [INSPIRE].

[23] M.S. Costa, V. Goncalves and J. Penedones, Conformal Regge theory, JHEP 12 (2012) 091 [arXiv: 1209.4355] [INSPIRE].

[24] A.V. Kotikov and L.N. Lipatov, Pomeron in the $N=4$ supersymmetric gauge model at strong couplings, Nucl. Phys. B $\mathbf{8 7 4}$ (2013) 889 [arXiv:1301.0882] [INSPIRE].

[25] R.C. Brower, M.S. Costa, M. Djurić, T. Raben and C.-I. Tan, Strong Coupling Expansion for the Conformal Pomeron/Odderon Trajectories, JHEP 02 (2015) 104 [arXiv:1409.2730] [INSPIRE].

[26] I. Balitsky, V. Kazakov and E. Sobko, Structure constant of twist-2 light-ray operators in the Regge limit, Phys. Rev. D 93 (2016) 061701 [arXiv:1506.02038] [InSPIRE].

[27] I. Balitsky, V. Kazakov and E. Sobko, Three-point correlator of twist-2 light-ray operators in $N=4 S Y M$ in BFKL approximation, arXiv:1511.03625 [INSPIRE].

[28] I. Balitsky, Operator expansion for high-energy scattering, Nucl. Phys. B 463 (1996) 99 [hep-ph/9509348] [INSPIRE].

[29] Y.V. Kovchegov, Unitarization of the BFKL Pomeron on a nucleus, Phys. Rev. D 61 (2000) 074018 [hep-ph/9905214] [inSPIRE].

[30] Y.V. Kovchegov, Small x F(2) structure function of a nucleus including multiple Pomeron exchanges, Phys. Rev. D 60 (1999) 034008 [hep-ph/9901281] [InSPIRE].

[31] G.P. Korchemsky, Conformal bootstrap for the BFKL Pomeron, Nucl. Phys. B 550 (1999) 397 [hep-ph/9711277] [INSPIRE]. 
[32] L.N. Lipatov, The Bare Pomeron in Quantum Chromodynamics, Sov. Phys. JETP 63 (1986) 904 [INSPIRE].

[33] A.R. White, The Triangle anomaly in triple Regge limits, Phys. Rev. D 63 (2001) 016007 [hep-ph/9910458] [INSPIRE].

[34] M.S. Costa, J. Penedones, D. Poland and S. Rychkov, Spinning Conformal Correlators, JHEP 11 (2011) 071 [arXiv:1107.3554] [INSPIRE].

[35] I. Balitsky, NLO BFKL and anomalous dimensions of light-ray operators, Int. J. Mod. Phys. Conf. Ser. 25 (2014) 1460024 [INSPIRE].

[36] I. Balitsky and G.A. Chirilli, High-energy amplitudes in $N=4$ SYM in the next-to-leading order, Phys. Lett. B 687 (2010) 204 [arXiv:0911.5192] [InSPIRE].

[37] I. Balitsky and G.A. Chirilli, NLO evolution of color dipoles in $N=4 S Y M$, Nucl. Phys. B 822 (2009) 45 [arXiv:0903.5326] [INSPIRE].

[38] M. Alfimov, N. Gromov and V. Kazakov, QCD Pomeron from AdS/CFT Quantum Spectral Curve, JHEP 07 (2015) 164 [arXiv: 1408.2530] [INSPIRE].

[39] L. Cornalba, Eikonal methods in AdS/CFT: Regge theory and multi-reggeon exchange, arXiv:0710.5480 [INSPIRE].

[40] I. Balitsky, Factorization and high-energy effective action, Phys. Rev. D 60 (1999) 014020 [hep-ph/9812311] [INSPIRE].

[41] I. Balitsky, High-energy QCD and Wilson lines, hep-ph/0101042 [INSPIRE].

[42] G.A. Chirilli and Y.V. Kovchegov, Solution of the NLO BFKL Equation and a Strategy for Solving the All-Order BFKL Equation, JHEP 06 (2013) 055 [arXiv:1305.1924] [INSPIRE].

[43] G.A. Chirilli and Y.V. Kovchegov, $\gamma^{*} \gamma^{*}$ Cross Section at NLO and Properties of the BFKL Evolution at Higher Orders, JHEP 05 (2014) 099 [Erratum ibid. 08 (2015) 075] [arXiv: 1403.3384] [INSPIRE].

[44] I.I. Balitsky and V.M. Braun, Nonlocal Operator Expansion for Structure Functions of $e^{+} e^{-}$ Annihilation, Phys. Lett. B 222 (1989) 123 [INSPIRE].

[45] I.I. Balitsky and V.M. Braun, The Nonlocal operator expansion for inclusive particle production in $e^{+} e^{-}$annihilation, Nucl. Phys. B 361 (1991) 93 [INSPIRE].

[46] I.I. Balitsky and V.M. Braun, Valleys in Minkowski space and instanton induced cross-sections, Nucl. Phys. B 380 (1992) 51 [INSPIRE].

[47] A. Babansky and I. Balitsky, Scattering of color dipoles: From low to high-energies, Phys. Rev. D 67 (2003) 054026 [hep-ph/0212075] [INSPIRE].

[48] I. Balitsky, Operator expansion for diffractive high-energy scattering, AIP Conf. Proc. 407 (1997) 953 [hep-ph/9706411] [INSPIRE].

[49] Y.V. Kovchegov and E. Levin, Quantum chromodynamics at high energy, vol. 33, Cambridge University Press (2012) [INSPIRE].

[50] V.A. Smirnov, Feynman integral calculus, Springer (2006) [INSPIRE]. 